\title{
High-Efficient Electrodes For Novel Optoelectronic Devices In Silicon Photonics
}

\section{Álvaro Rosa Escutia}

Supervisor: Dr. Pablo Sanchis Kilders

Tesis presentada para la obtención del grado de Doctor en Telecomunicaciones de la Universitat Politècnica de València.

Thesis submitted to the Universitat Politècnica de València in partial fulfillment of the requirements for the degree of Doctor of Philosophy in Telecommunications engineering. 

Al meu pare Àlvaro 

"Remember to look up at the stars and not down at your feet. Try to make sense of what you see and wonder about what makes the Universe exist. Be curious. And however difficult life may seem, there is always something you can do and succeed at. It matters that you don't just give up."

Professor Stephen W. Hawking 



\section{Acknowledgments}

Primero de todo, quiero dar las gracias a Pablo por escucharme por primea vez, cuando halla por 2009 buscaba proyecto final de carrera en Gandía, ese momento me ha llevado hasta escribir hoy estas líneas. También por confiar en mi para realizar el doctorado, por sus consejos y su orientación, y por haberme dado libertad para trabajar y escuchar siempre mis ideas, algunas veces acertadas y otras no tanto. No ha sido un camino fácil, pero he disfrutado y aprendido muchísimo, y por ello, estaré siempre agradecido.

En este camino me he cruzado con personas maravillosas con las cuales he compartido este increíble camino, y a ellas quiero darles las gracias también. A Marghe, Pau, Luis y Sergio, por haber sido un pilar tanto profesional como personalmente. A Anita, por haber sido una compañera de Padel estupenda, mejor "jefa" y amiga. A Raffaele, por haber sufrido juntos en el gimnasio y fuera, en el deporte y en el trabajo. A Andrés, por tener a un madridista al que pinchar, a un rival en la pista de padel y a un amigo fuera de ella. A Fran, Andrea, Kike, Alba, Ángela, Luis bio, Jad, Diego y Alex gracias por compartir vuestro tiempo conmigo. A Antoine y Domenico, por tener siempre un momento para echar una mano, y siempre con una sonrisa. A todo el equipo de Fabricación y encapsulado, Amadeu, Jose, Aina, Dora, Juan, Laurent y Cristina, por vuestra profesionalidad y simpatía. A otros pabsankis como yo, Irene, Roberto o Jorge, gracias y animo con vuestras tesis, el esfuerzo merece la pena.

I am also thankful to my supervisor in Ghent University, Dries Van Thourhout, and to my workmates. You all made me felt at home and I will be always grateful.

Als amics del poble, Alginet, que sempre s'han mostrat orgullosos i m'han animat i recolzat en el meu camí, encara que això haja suposat veurem alguna vegada menys el pèl. Eixe suport no te preu. L'WQM no te preu!

I finalment, no podria estar mes agraït a la meua família. Al meu germà, per tots el bons moments i per tot el que aprenc amb ell, però sobretot per la seua paciència amb mi els últims mesos de doctorat, on estava un poc mes nerviós del normal. Als meus pares, per haver sigut un exemple de fortalesa, superació i bondat. Per haver confiat sempre en mi, sobretot de menut, quan era un poc més revoltós de lo normal i les meues notes no eren sempre les esperades. Sou en meu pilar mestre i no podria tindre una família millor. 


\section{Resum}

La fotònica de silici és actualment la tecnologia millor posicionada per a reemplaçar les connexions electròniques tant dins del propis xips, com entre ells mateixos, amb la finalitat de millorar el seu rendiment. Els principals avantatges de la tecnologia fotònica de silici resideixen en el seu baix cost i en la seua compatibilitat amb les actuals tècniques de fabricació desenvolupades per la indústria microelectrònica. Aquesta compatibilitat permetria fabricar tant xips òptics com a xips híbrids que incloguen components òptics i electrònics. Els moduladors i els commutadors optoelectrònics resulten dispositius fonamentals en aplicacions de telecomunicacions. Les principals funcions dels commutadors i moduladors optoelectrònics són l'encaminament i la transmissió de dades d'alta velocitat.

Aquesta tesi aborda el disseny i l'optimització de la part elèctrica i òptica (en menor mesura) amb la finalitat d'optimitzar el rendiment de tals dispositius des del punt de vista optoelectrònic. A més, també es tractarà la introducció de nous materials compatibles amb el silici i els seus processos de fabricació, com el diòxid de vanadi o el titanato de bari amb la finalitat de demostrar les seues propietats i aplicar-les als dispositius optoelectrònics amb la finalitat de millorar el seu rendiment. 


\section{Resumen}

La fotónica de silicio es actualmente la tecnología mejor posicionada para reemplazar las conexiones electrónicas tanto dentro de los mismos chips, como entre ellos mismos, con el fin de mejorar su rendimiento. Las principales ventajas de la tecnología fotónica de silicio residen en su bajo coste y en su compatibilidad con las actuales técnicas de fabricación desarrolladas por la industria microelectrónica. Dicha compatibilidad permitiría fabricar tanto chips ópticos como chips híbridos que incluyan componentes ópticos y electrónicos. Los moduladores y los conmutadores optoelectrónicos resultan dispositivos fundamentales en aplicaciones de telecomunicaciones. Las principales funciones de los conmutadores y moduladores optoelectrónicos son el enrutamiento y la transmisión de datos de alta velocidad.

Esta tesis aborda el diseño y la optimización de la parte eléctrica y óptica (en menor medida) con el fin de optimizar el rendimiento de tales dispositivos desde el punto de vista optoelectrónico. Además, también se tratará la introducción de nuevos materiales compatibles con el silicio y sus procesos de fabricación, como el dióxido de vanadio o el titanato de bario con el fin de demostrar sus propiedades y aplicarlas a los dispositivos optoelectrónicos con el fin de mejorar su rendimiento. 


\section{Abstract}

Silicon photonics is nowadays the most promising technology to replace electrical inter- and intra-connections of the chips, increasing the performance in this way. The main advantages of silicon photonics technology lie on its low cost and its compatibility with the fabrications processes of microelectronics industry developed during years which allows the mass production of silicon photonics chips as well hybrid electronic and photonic devices in the same chip. Optoelectronics switches and modulators are key building blocks in photonic devices for tele/datacom applications. Such switches and modulators are devices which provides routing functionalities and the transmission of high speed data respectively.

The work of this thesis delves with the design and optimization of silicon based switches and modulators spotlighting the electrical elements. Additionally, the work of this theses deals with the introduction of new silicon-compatible materials as vanadium dioxide and barium titanate, with the aim of demonstrating its functionalities and develop high-performance optoelectronic devices. 


\section{Table of contents}

Acknowledgments ...................................................

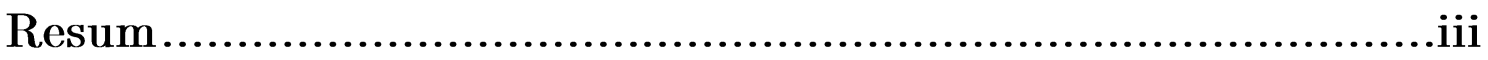

Resumen .................................................................

Abstract..................................................................... vii

Table of contents.......................................................

1. Introduction ..........................................................

1.1 A brief history of silicon photonics.............................................. 1

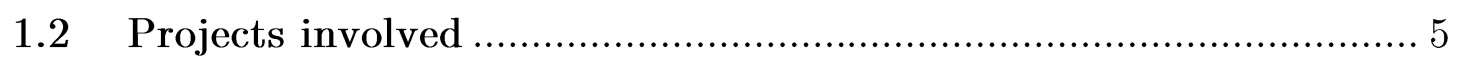

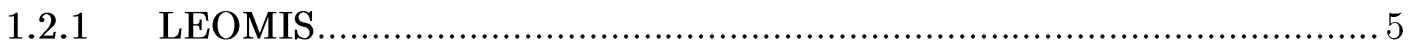

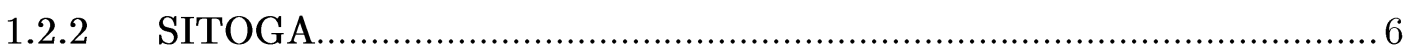

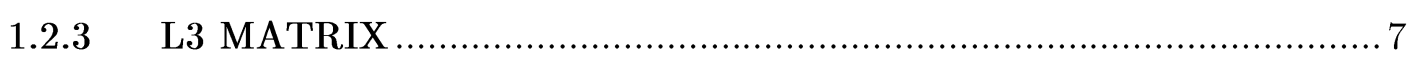

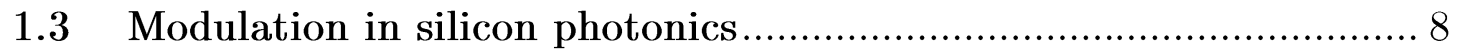

1.3.1 Physical modulation mechanisms ................................................... 9

1.3.1.1 Pockels effect......................................................................... 10

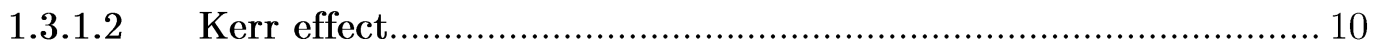

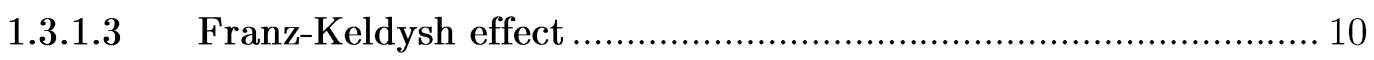

1.3.1.4 Plasma dispersion effect......................................................... 11

1.3.1.5 Thermo-optic effect.................................................................... 11

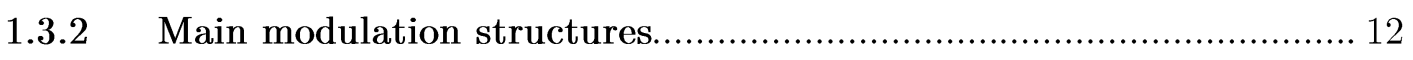

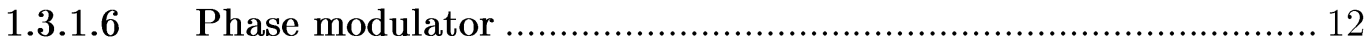

1.3.1.7 Amplitude modulators ........................................................ 12 


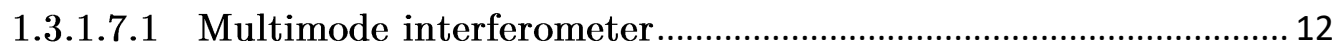

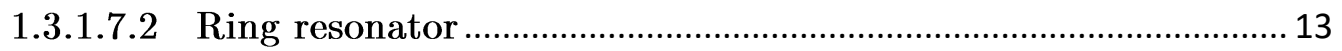

1.3.1.7.3 Mach-Zehnder Interferometer (MZI) modulator .......................... 14

1.3.1.7.4 Traveling-wave modulator.......................................................... 15

1.4 Objectives and outline of the thesis ................................... 17

2. High-efficient micro-heaters design for application in

photonic switches ..................................................... 19

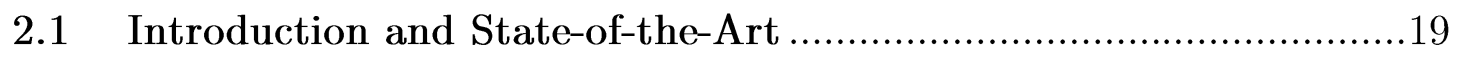

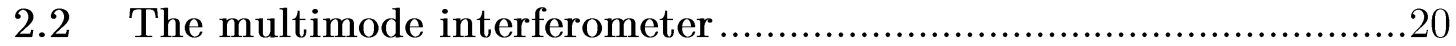

2.3 Tunable multimode interferometer design .............................22

2.4 Thermal optimization of the multimode interferometer.................24

2.5 Micro-heater design for multimode interferometers .....................29

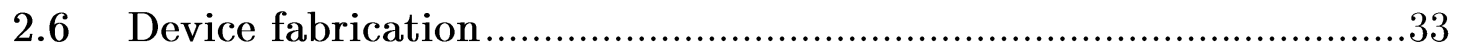

2.7 Characterization and experimental results ................................ 34

2.8 Application of micro-heater structures in $\mathrm{VO}_{2}$-silicon based switches 39

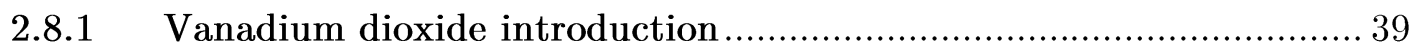

2.8.2 Device description and method analysis ..................................... 40

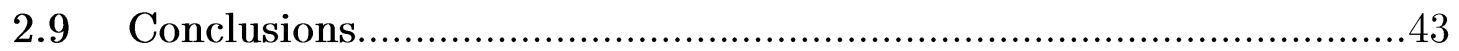

3. Novel RF electrodes for application in optoelectronic traveling wave modulators......................................... 45

$3.1 \quad$ Introduction and State-of-the-Art ..............................................45

3.2 Coplanar waveguide theory .................................................46

3.2.1 Transmission line theory and background ................................. 46

3.2.2 Conformal mapping method …............................................. 50

$3.3 \quad$ Measurement methods ....................................................... 54

3.3.1 Microwave index extraction through ABCD matrix ........................ 54

3.3.2 Microwave index extraction through multiline method .................. 56

3.4 Design of slow-wave coplanar waveguides................................58

3.5 Fabrication and characterization of slow-wave coplanar waveguides 65 
3.6 Conclusions.

4. RF electrodes design for novel BTO-Si electro-optic modulators 69

4.1 Introduction and State-of-the-Art 69

4.2 Relative permittivity extraction of Barium Titanate...................71

4.3 Electrode and electro-optical design of BTO-Si based modulator...77

4.4 Characterization of BTO-Si based modulator ........................... 81

4.4.1 Electro-optical set-up and final results ..................................... 82

4.5 Conclusions........................................................................ 86

5. Conclusions and future outlook ............................... 87

List of publications........................................... 89

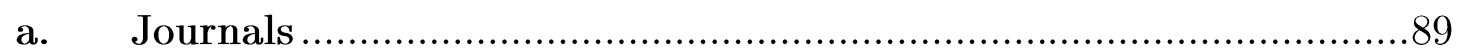

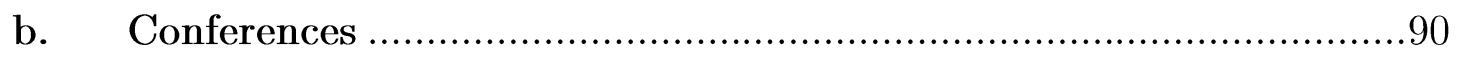

References ...................................................... 93 


\section{Chapter 1}

\section{Introduction}

\subsection{A brief history of silicon photonics}

Photonics involves the generation, manipulation and detection of photons in two independent ways: as single particles or as electromagnetic waves. In addition, photonics as it is known today, starts with two characteristic inventions: the optical fiber in 1950 as an endoscope with medical purposes, and the laser as a tool for experimental physics in 1960.
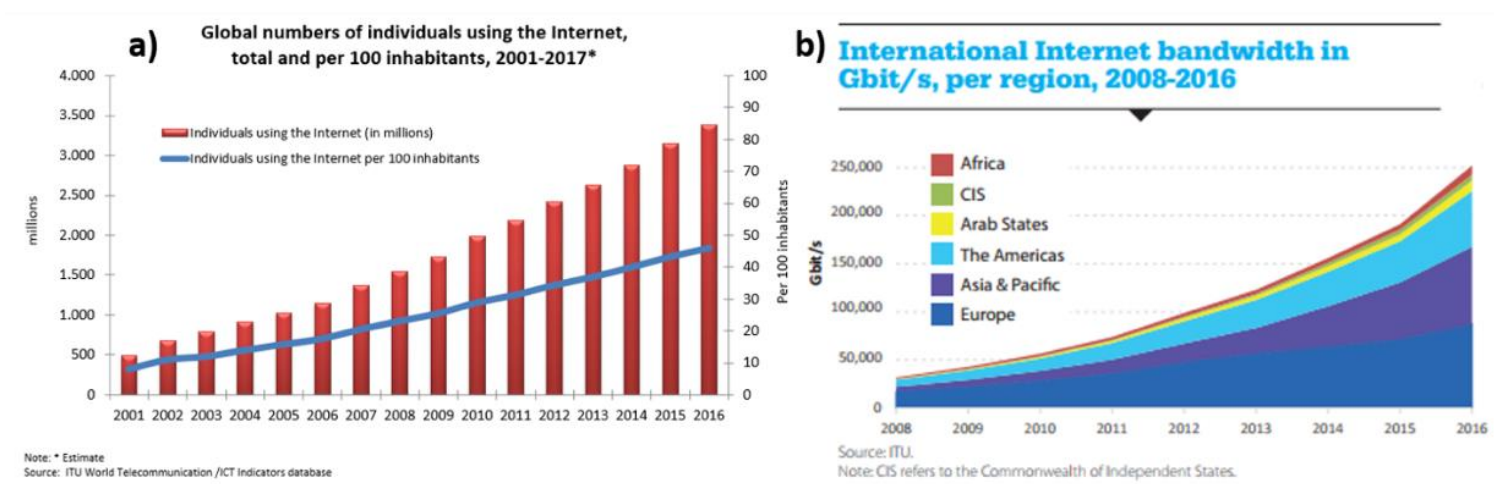

Figure 1. (a) Total number and percentage of internet users around the world. From 2002 to 2016. (b) International internet bandwidth demand from 2008 to 2016 [1].

In the last years, the requirements of the communications networks have been growing due to the increasing demand. Such increasing demand is related with the rise of the number of users and the required quality of new services [1]. 
To face the challenge of networks rising requirements, an evolution from electrical to optical networks becomes crucial [2]. Optical fiber communications have overwhelmed the performance of commonly electrical communications due to different reasons. One of them is the low losses in long range communications. Another reason is the immunity to the electromagnetic interferences, heating noise or crosstalk. Finally, optical communications allow higher data rates because the optical fiber bandwidth is much higher than any other electrical medium.

The first step to carry on the network evolution was to replace the electrical cooper transmission lines by optical fibers. However, system nodes remain working in electrical domain. In this context, the emergence of inter- and intra-chip optical interconnects through integrated photonic devices is gradually expected to overwhelm copper based-interconnects. As a result, significant research efforts have been made to realize low drive voltage, low power and fast switching devices featuring high throughput bandwidth. In this context, silicon photonics is the most promising technology to develop the future photonic devices for short range communications, intra- and even in on-chip scale communications [3].

During last years, silicon has become the key material in electronics and photonics industry. The revolution in the electronic industry was intrinsically connected to the transistor development on semiconductor materials. The transistor was the solution to replace electrons tube, which was used for amplifying electrical signals in long range communications. In this context, a new transistor was developed as a silicon-based integrated device. Its main properties, like electrical amplification and bistability made it suitable to develop multi-operational electronic chips. That was the beginning of one of the most important technological races in the $20^{\text {th }}$ century, reducing the transistor's size as well as its dissipated heat in order to increase the number of transistor per chip and therefore, increase its functionality and its computation capacity. Thus, the electronic industry developed itself around the silicon-based transistor and its fabrication process called complementary metal-oxide-semiconductor (CMOS), which enables the mass production and reduces the price per chip and therefore the price of final electronic devices (see Table 1).

Regarding to the photonics industry, silicon has many advantages that makes it suitable for the development of photonics components. Thus, it is compatible with the electronics industry which reduces its production costs by using all the infrastructure already available and allows the design of hybrid technology including both photonic and electronic devices in the same chip. Furthermore, it 
is one of the most abundant elements around the world, which makes silicon one of the cheapest available materials. To develop silicon photonic devices, silica $\left(\mathrm{SiO}_{2}\right)$ is one the key material for the process. The high index contrast between silicon (3.45) and silica (1.45) enables a strong light confinement which benefits the size reduction of the devices to the nanometer scale allowing optical devices with ultra-compact foot-prints [4]. The mixing layer technology of silicon and silica to perform electronic and photonic devices is called silicon-on-insulator (SOI). It is easily processable due to the development of the electronics industry around silicon.

Table 1 shows the cost fabrication for a simple and complex chip as a function of the fabrication volume per year.

\begin{tabular}{|c|c|c|}
\hline & Simple chip $(€)$ & Complex chip $(€)$ \\
\hline $\begin{array}{c}\text { Very low volume } \\
(100-1000 \text { per year })\end{array}$ & 100 & 1000 \\
\hline $\begin{array}{c}\text { Low volume } \\
(<10 \text { k per year })\end{array}$ & 10 & 10 \\
\hline $\begin{array}{c}\text { Medium volume } \\
(10 \mathrm{k}-1 \text { M per year })\end{array}$ & 1 & 1 \\
\hline $\begin{array}{c}\text { High volume } \\
(>1 \text { M per year })\end{array}$ & 0.1 & Ph \\
\hline
\end{tabular}

Table 1. Chip cost fabrication in a CMOS process. Data obtained in the ePIXfab Silicon Photonics training course at the European Conference in Optic Communications (ECOC) 2015.

However, despite silicon is a low cost and well-studied material with good thermal and electrical properties and offers the possibility of creating waveguiding structures with SOI technology, there are some disadvantages to develop silicon photonic devices. The main points that silicon photonics technology has to deal with are presented in the next lines:

- The indirect silicon bandgap restricts the light-emission and thus the development of optical sources.

- Due to its centro-symmetric crystallography, silicon has no Pockels coefficient, which prevents its refractive index modulation.

- The efficiency of pure silicon photodetectors at communication wavelengths of 1310 and $1550 \mathrm{~nm}$, makes them inappropriate for high speed and high responsivity operation.

Thus, to carry out such challenges and develop multifunctional photonic chips, the integration of other materials compatible with silicon as well as CMOS 
fabrication process is one of the scientific pursues nowadays. Some materials can overcome some silicon properties to develop photonic devices, such as some of III$\mathrm{V}$ compounds like indium phosphide (InP) or ferroelectric materials like lithium niobate $\left(\mathrm{LiNbO}_{3}\right)$ or barium titanate $\left(\mathrm{BaTiO}_{3}\right)$.

On one hand, germanium is one of the most used material for developing photodetectors on silicon integrated photonic devices due to its excellent properties of light absorption and its compatibility with CMOS fabrication process [5].

On the other hand, III-V compounds have been widely investigated for solving the challenge of light emission in silicon photonics structures [6],[7]. Its lasing properties makes them suitable for the development of light sources, although the challenge of the mass fabrication still remains due to the lack of compatibility with the CMOS process.

Furthermore, silicon has no pockels effect and therefore it is not possible to use the linear electro-optic effect in order to implement switching or modulating functionalities. In this way, quadratic electro-optic effect is too weak to be used as a modulating effect in silicon. Fortunately, silicon has a good thermo-optic coefficient which can be used to modulate the refractive index. Unfortunately, thermo-optic effect is a relatively slow effect and then it is only suitable for switching applications. The integration of transition metal oxides (TMO) in silicon is one of the most promising options for the implementation of EO functionalities, especially high-speed modulation, beyond state-of-the art, which cannot be afforded currently in silicon photonics technology itself. TMO materials comprise a very large class of compounds with unique properties that can be tailored for a wide range of applications.

As it can be seen, the scientific community and electronic industry are looking photonics [8] as an option to replace and/or overwhelm some electrical devices and functionalities. Such complex and multifunctional photonic chips are composed by several single optoelectronic devices.

Both electrical and optical elements of an optoelectronic device need to be well studied and optimized to obtain the maximum performance. This thesis has been focus on the development and optimization of such electrical elements. Since this is a transversal topic in photonics technology with application in different optoelectronic devices, the work of this thesis has been involved in different European and national projects. 
To summarize, the work developed on this thesis will address theoretically and experimentally the study, development and optimization of the electrical elements with different functionalities for different optoelectronic devices.

\subsection{Projects involved}

As it has been aforementioned, the work developed in this thesis has been involved in the framework of different European and national research projects: the national project LEOMIS (Light emitting and electro-optical integrated devices in silicon photonics CMOS technology. Towards disruptive and smart silicon solid state lighting and functional optical interconnects) and the European projects SITOGA (Silicon CMOS compatible transition metal oxide technology for boosting highly integrated photonic devices with disruptive performance) and L3MATRIX (Large Scale Silicon Photonics Matrix for Low Power and Low-Cost Data Centers).

\subsubsection{LEOMIS}

The LEOMIS project (TEC2012-38540-C02-02) aimed to contribute to the expansion of Silicon CMOS photonics by enabling an integrated design, fabrication, assembling, testing and packaging chain in this field. This has been validated with the development of state of the art silicon optoelectronic devices:

- Silicon-based light emitting diodes for smart silicon solid state lighting (vertical cavity) and optical interconnects (horizontal cavity).

- Electro-optical modulators and switching devices for on-chip routing and optical interconnects.

The LEOMIS project gathers two influential groups in the field of silicon CMOS photonics in Spain: The Department of Electronics of University of Barcelona (UB) and the Nanophotonics Technology Center of the Polytechnic University of Valencia (UPV). 


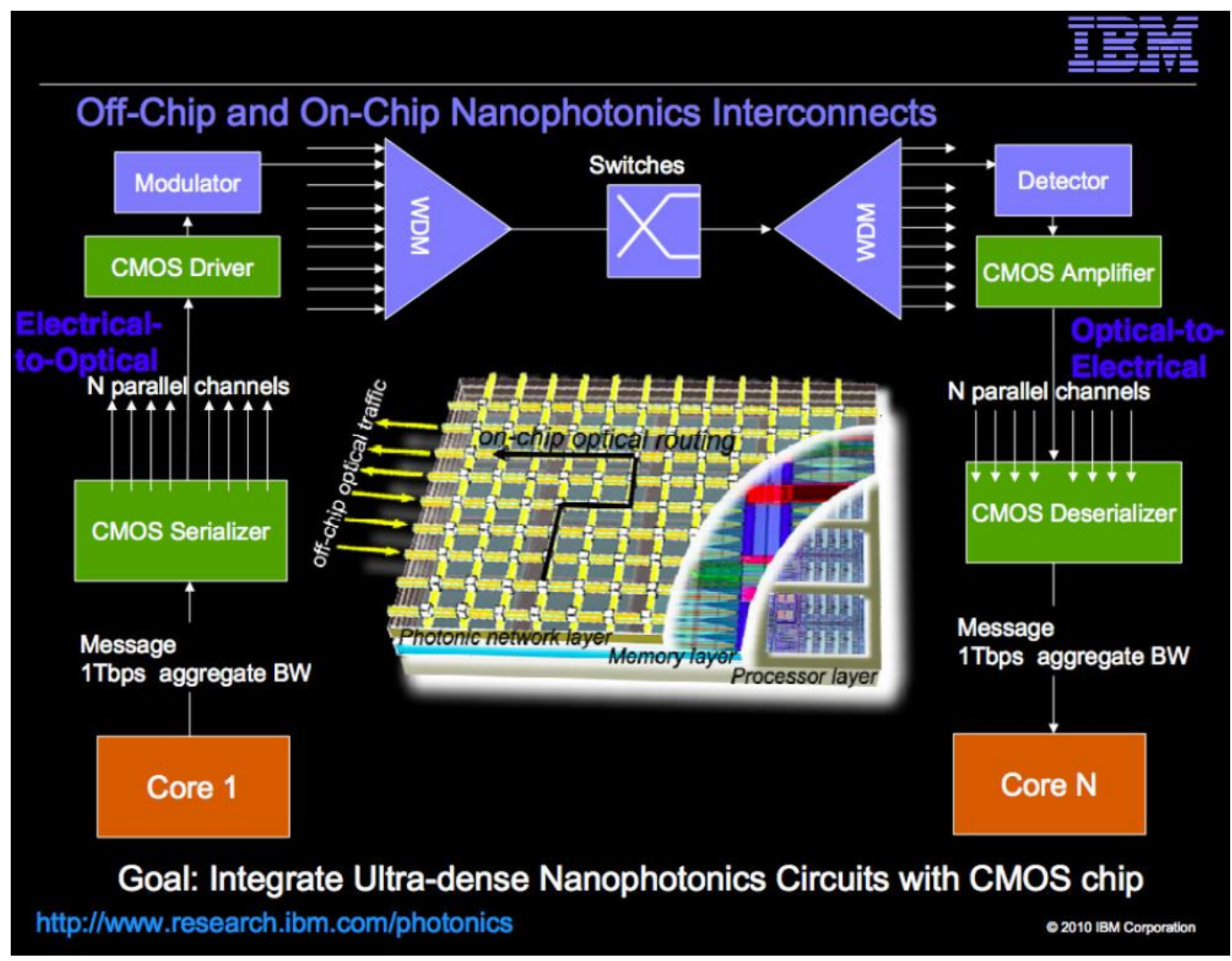

Figure 2. Scheme of optical intra-chip routing proposed by IBM (ePIXfab Silicon Photonics training course at the European Conference in Optic Communications (ECOC) 2015).

Regarding to the project LEOMIS this thesis has contributed with the design and optimization of SOI optoelectronic switches based on tunable multimode interferometers (MMI) through thermo-optic effect, focused on the reduction of the power consumption and demonstrating the switching functionality.

\subsubsection{SITOGA}

By other hand, the SITOGA project (FP7-ICT-2013-11-619456) addressed the integration of transition metal oxides (TMO) materials in silicon photonics technology for offering breakthrough electro-optical functionalities due to their unique properties not present in pure silicon. Such integration combined with the development of beyond state-of-the art photonic devices have shown the way towards a wide range of photonic applications. Figure 3 summarizes the SITOGA project concept. The consortium of the project was composed by the Universitat Politecnica de Valencia (UPVLC) in Spain, which was the coordinator, the Centre National de la Recherche Scientifique-Institut des Nanotechnologies de Lyon (CNRS-INL) in France, the Katholieke Universiteit Leuven (KUL) in Belgium, 
the Leibniz Institute for Innovative Microelectronics (IHP) in Germany, IBM Research GmbH in Switzerland and DAS Photonics in Spain.

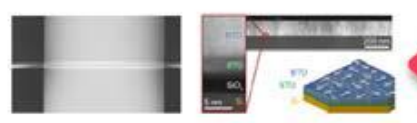

$\mathrm{VO}_{2}$ on $\mathrm{Si}$

INNOVATIVE MATERIALS

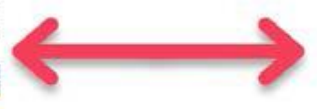

EO modulators

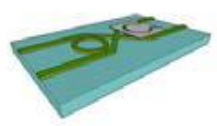

EO switches

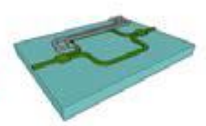

BEYOND STATE-OF-THE-ART PHOTONIC DEVICES

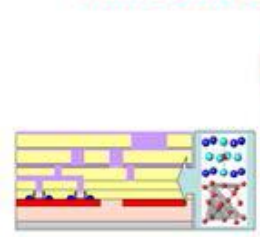

CMOS electronics integration

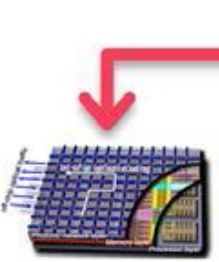

Computing
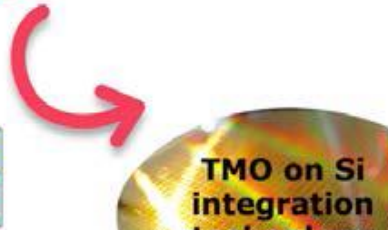
technology

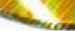

LOW-COST VOLUME MANUFACTURING

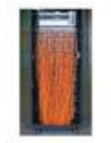

Datacom

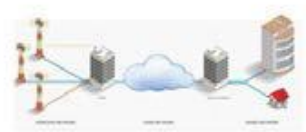

Telecom

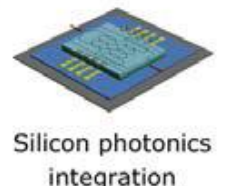

integration

WIDE RANGE OF APPLICATIONS

Figure 3. Summary of SITOGA project concept, which consist on mixing silicon photonics with TMO properties.

The work of this thesis involved in the SITOGA project was focused on the electrical design of micro-heaters for application in vanadium dioxide $\left(\mathrm{VO}_{2}\right)$ silicon-based switches and the electrical characterization of barium titanate ( $\mathrm{BaTiO}_{3}$ or BTO) to properly design the modulator electrodes, and therefore, the BTO-Si based electro-optical modulator.

\subsubsection{L3 MATRIX}

Finally, the L3 MATRIX project (H2020-ICT-27-2015-688544) provides novel technological innovations in the fields of silicon photonics and 3D device integration. The project is developing a novel silicon photonics matrix with a scale larger than any similar device with more than 100 modulators on a single chip and will integrate embedded laser sources with a logic chip thus breaking the limitations on the bandwidth-distance product. 


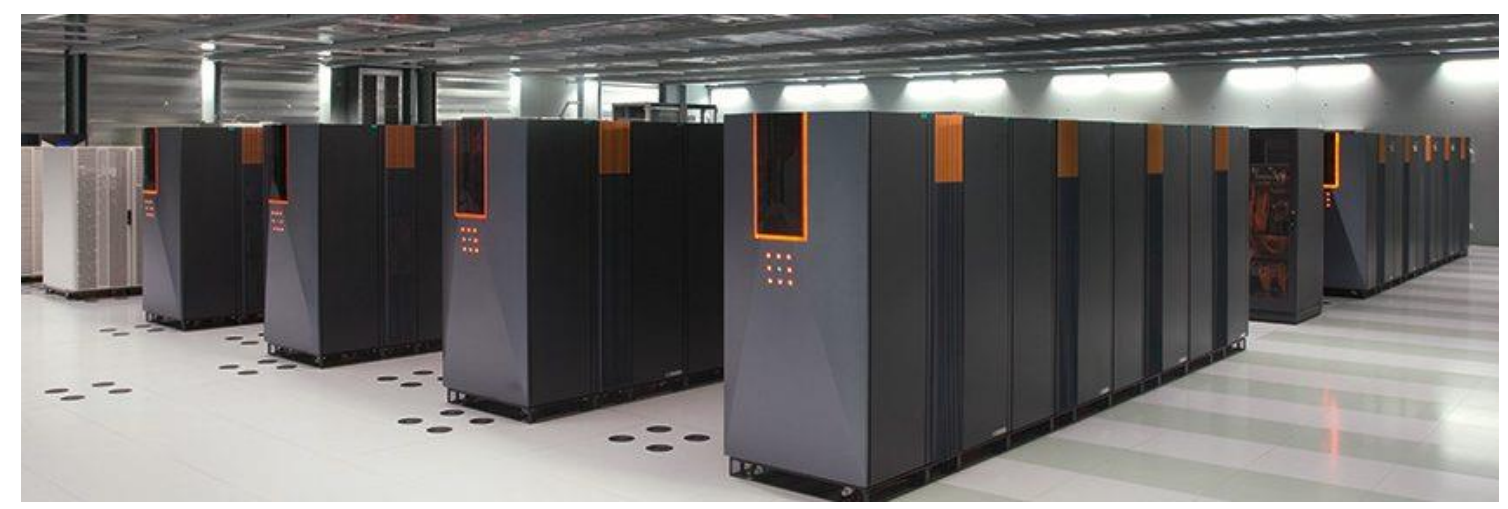

Figure 4. Data centers are the main target for the implementation of the L3 MATRIX devices. (http://13matrix.eu/)

A novel approach is used with embedded III-V sources on the SOI substrate which will eliminate the need to use an external light source for the modulators. L3 MATRIX presents a new method of building switching elements and provides an extended bandwidth of $25 \mathrm{~Gb} / \mathrm{s}$ in single mode fibers and waveguides with low latency. The power consumption of DC networks built with these devices is 10fold lower compared to the conventional technology. The outcome of this approach is that large networks, in the $\mathrm{Pb} / \mathrm{s}$ scale can be built as a single stage, non-blocking network.

The work of this thesis within L3MATRIX project was focused on the development of slow-wave electrodes for application in mach-zehnder interferometer (MZI) modulators or switches based on slow-wave structures for a near future application in the large-scale matrix data centers. Slow-wave electrooptic modulators have been reported to reduce the drive voltage and footprint [9]-[12]. However, slow wave electrodes become essential for matching microwave and optical indices, and then to avoid a reduction of the electro-optic modulation bandwidth [13].

\subsection{Modulation in silicon photonics}

Two of the most important workhorses in photonic integrated circuits are optical modulators and switches, which share physical mechanism and structures [14]. Optical modulators are used to transform an electrical signal into an optical signal, with the purpose to be transmitted through optical links meanwhile optical switches is used to guide optical data transmissions into one path or another.

The modulation effect is produced by an external perturbation (the signal that is going to be transmitted) which causes a change in the properties of the photonic 
structure. That change is reflected on the transmitted optical signal (Figure 5 shown an amplitude modulation). Due to the existence of different kinds of physical changes and different kinds of structures, a modulator can be classified in different ways. Regarding to the structure type, modulators can be classified as amplitude modulators, phase modulators or polarization modulators. Furthermore, classifying by the physical effect there are 4 possible groups: electrooptic modulators, electro-absorption modulators, carrier injection or depletion modulators and thermo-optic modulators. Figure 5 illustrates an example of amplitude modulation concept.

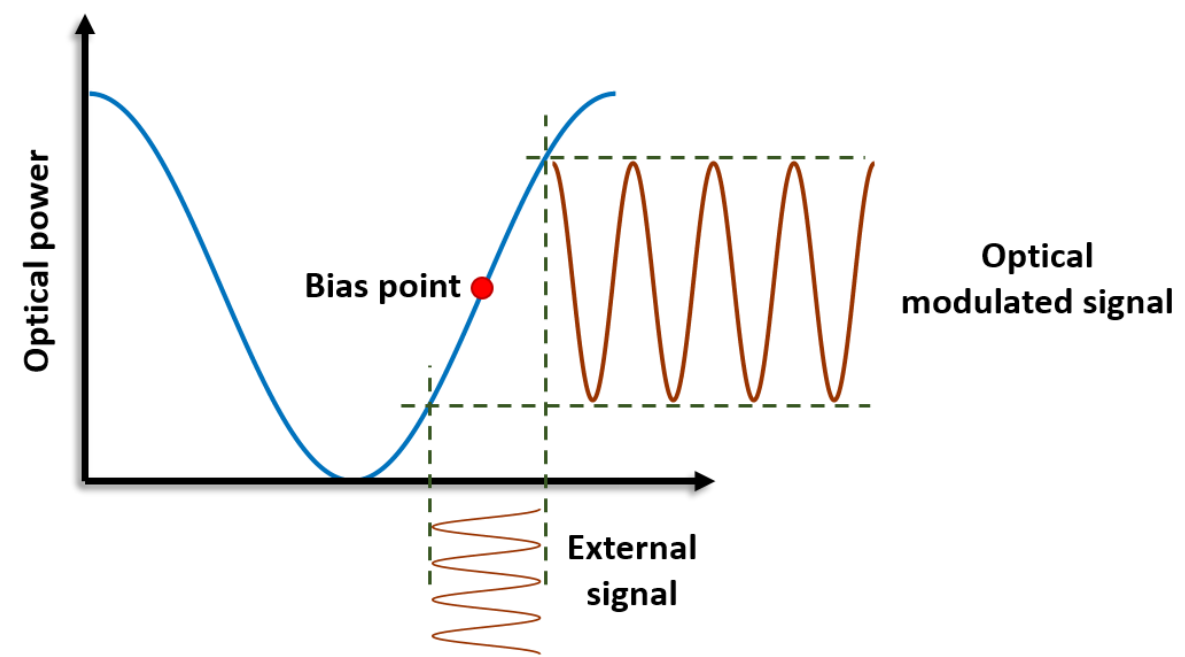

Figure 5. Optical modulator operation example. The external signal is the perturbation which produces de modulation effect. The external signal produces de optical modulated signal and its amplitude depend of the amplitude of the external signal and the bias point.

\subsubsection{Physical modulation mechanisms}

The changes in the refractive index and the absorption coefficient of the material are the main physical effects used in optical modulators. On one hand, modulators based on refractive index changes are known as electro-optical modulators and, on the other hand, modulators based on absorption coefficient changes are known as electro-absorption modulators [15]. The refractive index is defined as a complex number with real and imaginary parts. The real part represents the refractive index as it is commonly known, and the imaginary part represents the material absorption. The application of an electrical field could lead to a change in both parts of the refractive index. When the change is produced only in the real part, the effect is known as electro-refraction. However, when the change is produced in the complex part, the effect is known as electro-absorption. Here, a brief description of the main electro-optic effects is presented: 


\subsubsection{Pockels effect}

This effect causes a change in the real part of the refractive index, being proportional to the applied electric field intensity. Furthermore, the Pockels effect generates a change in the direction of the applied electric field respect to the material crystallographic axes. Therefore, the Pockels effect is polarization dependent. Since the silicon geometry of crystallographic structure makes Pockels effect ineffective, this effect cannot be used to develop silicon-based modulators. Nevertheless, it is widely useful in other materials like lithium niobate (LiNO3) or barium titanate (BaTiO3). The resulting index change is expressed in the following equation:

$$
\Delta n=-r_{i j} n_{i j} \frac{E}{2}
$$

where $E$ is the applied electric field, $r_{i j}$ is the Pockels coefficient and $n_{i j}$ is the corresponding refractive index.

\subsubsection{Kerr effect}

Kerr effect is also known as quadratic electro-optic effect. It changes the real part of the refractive index with the square of the electric field applied. The Kerr effect is present in silicon, unlike Pockels effect. However, it is relatively weak to be used for a modulator development. The Kerr effect behavior is described by Eq. $(1.2)$ :

$$
\Delta n=s_{i j} n_{0} \frac{E^{2}}{2}
$$

where $\mathrm{E}$ is the applied electric field, $s_{i j}$ is the Kerr coefficient and $n_{0}$ is the unperturbed refractive index. As can be seen in Eq. (1.2), the square of the electric field makes the refractive index variation polarization independent.

\subsubsection{Franz-Keldysh effect}

This effect is also known as electro-absorption effect, and is resulting of the energy band distortion of the semiconductor when an electric field is applied. This electric field shifts the bandgap energy, causing a change in the absorption properties of the crystal, particularly at wavelengths close to the bandgap and therefore a change in the complex refractive index. 


\subsubsection{Plasma dispersion effect}

This effect is based on the free carrier concentration variation in a semiconductor, which results in a modification of both real and imaginary parts of the refractive index. Soref and Bennett studied and evaluate the refractive index change and produce experimentally absorption curves for a wide range of electrons and holes densities [16]. Finally, the changes for a variation in the refractive index and absorption were quantified. As a result, the Eq. (1.3) and Eq. (1.4) were developed, which are widely used to evaluate such changes in silicon. Eq. (1.3) describes the variation in the real part of the refractive index as:

$$
\Delta n=\Delta n_{e}+\Delta n_{h}=-\left[8.8 \times 10^{-22} \Delta N_{e}+8.8 \times 10^{-18}\left(\Delta N_{h}\right)^{0.8}\right]
$$

where $\Delta n_{\mathrm{e}}$ is the change in the real part of the refractive index due to the change in the free electrons concentration and $\Delta n_{\mathrm{h}}$ is the change in the real part of the refractive index due to the change in the hole carrier's concentration. Finally, $\Delta N_{\mathrm{e}}$ and $\Delta N_{\mathrm{h}}$ are the concentration variation of electrons and holes in $\mathrm{cm}^{-3}$ respectively. Finally, Eq. (1.4) describes the variation in the imaginary part of the refractive index as:

$$
\Delta \alpha=\Delta \alpha_{e}+\Delta \alpha_{h}=8.5 \times 10^{-18} \Delta N_{e}+6.0 \times 10^{-18} \Delta N_{h}
$$

where $\Delta \alpha_{\mathrm{e}}$ is the change in the complex part of the refractive index due to the change in the free electrons concentration and $\Delta \alpha_{\mathrm{h}}$ is the variation in the complex part of the refractive index due to the change in the hole carrier's concentration. Both (1.3) and (1.4) equations were developed for a wavelength of 1550nm, which is the used wavelength for telecommunications.

\subsubsection{Thermo-optic effect}

It is based on the variation of the real part of the refractive index due to a temperature increase. In this case, silicon is a good candidate since it has a high thermo-optic coefficient:

$$
\frac{d n}{d T}=1.86 \times 10^{-4} / K
$$


However, it is a slow effect which makes it inappropriate for high speed modulators. Despite its slow response, thermo-optic effect is suitable for developing photonic switches with relaxed speed requirements. The development of high-efficient micro-heaters and thermo-optic switches will be discussed in the chapter 2 of this thesis.

\subsubsection{Main modulation structures}

Different structures can be used for developing optical modulators. The election of the appropriate structure depends on the required characteristics like modulation speed, insertion losses, device footprint, driving voltage and power consumption. Next, the main modulation structures and its basic principles are presented.

\subsubsection{Phase modulator}

A phase modulator is composed by single straight waveguides in which the refractive index is modified by an external signal. In this way, the resultant electric field is described in the next equation:

$$
E(z, t)=E_{0} \cos (\omega t-\beta z)=E_{0} \cos \left(\omega t-\frac{2 \pi}{\lambda_{0}} n(t) z\right)
$$

where $n(t)$ is:

$$
n(t)=n_{0}+\Delta n=n_{0}+K v(t)
$$

being $K$ a proportional constant which depends on the physical method used to modify the refractive index and $v(t)$ is the modulating signal.

\subsubsection{Amplitude modulators}

\subsection{Multimode interferometer}

A multimode interferometer (MMI) is a photonic structure composed by a multimode waveguide in which the total number of modes allowed by the physical characteristics of the waveguide are excited at the same time. When these modes begin to propagate trough the waveguide they are interfering with among them, generating the interference pattern. Self-images is the name given to the constructive interferences of the interference pattern. 


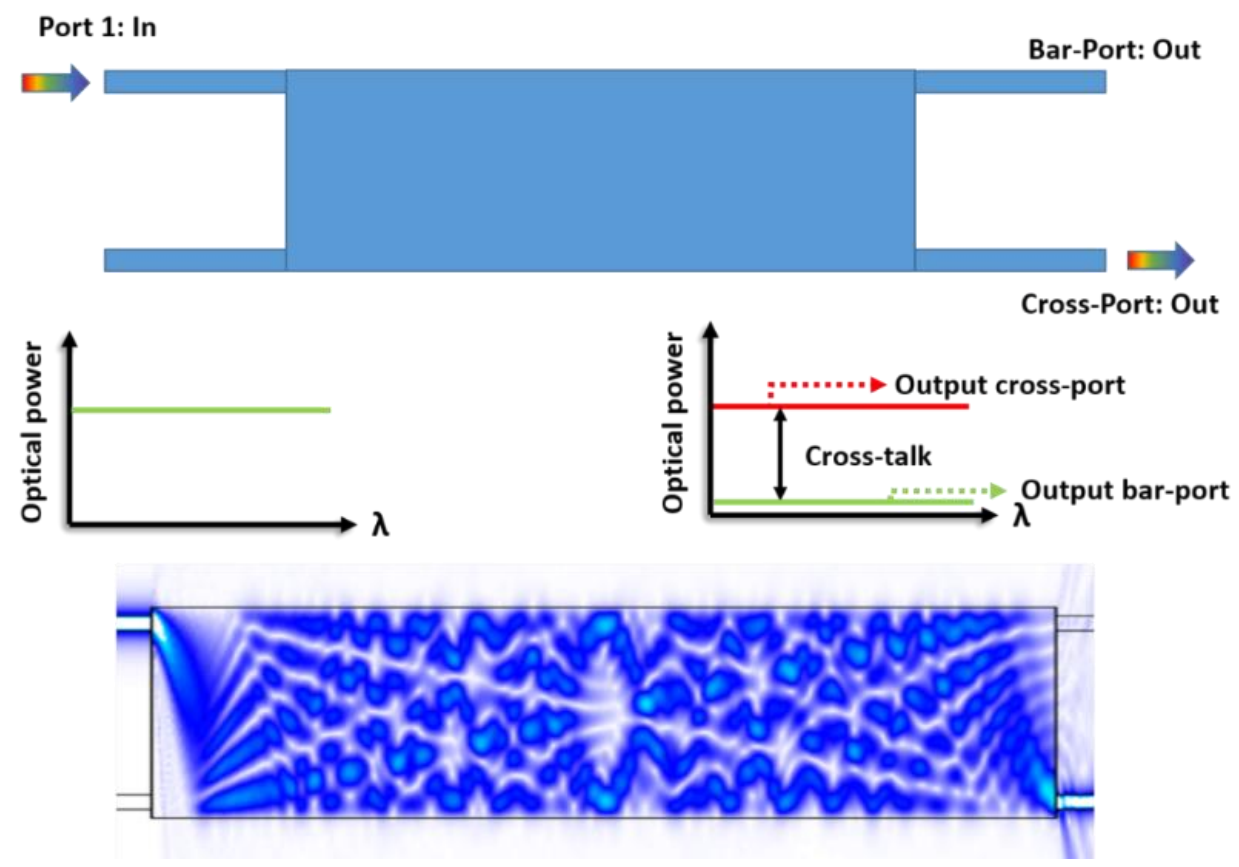

Figure 6. Multimode interferometer structure: its main parameters and a schematic of $1 \times 1$ configuration with a simulation image with the interference pattern.

The MMI becomes an amplitude modulator structure when a phase shift is applied over one of the two central self-images (see Figure 6). Thus, the optical output power in the cross-port is decreasing and, in the bar-port increasing. The concept of a tunable MMI will be discussed in detail along the chapter 2 of this thesis.

\subsection{Ring resonator}

A ring resonator is a photonic structure composed by a straight waveguide and a ring waveguide, placed close to each other, in which the light is guided through the straight waveguide and the resonance wavelength is coupled into the ring waveguide. Figure 6 shows a ring resonator configuration with its main parameters.

The resonance appears when the propagation length of the ring is an exactly whole number of wavelengths, as shown in the next equation:

$$
n_{\text {eff }} L_{\text {ring }}=M \lambda_{\text {res }}
$$

where $n_{\text {eff }}$ is the effective index of the waveguide, $L_{\text {ring }}$ is the length of the ring, $M$ is a whole number and $\lambda_{\text {res }}$ is the resonance wavelength. 
Port 1: In

Port 2: Out

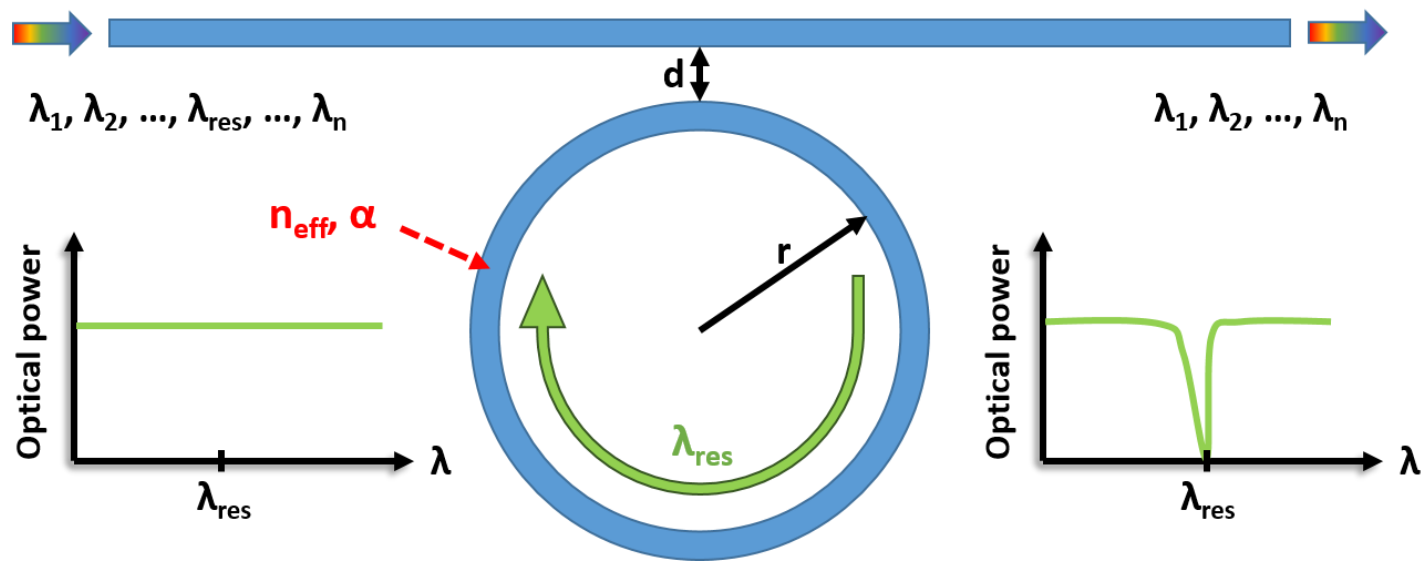

Figure 7. Ring resonator schematic with the most important design parameters.

Modulation in a ring resonator is achieved when the resonant wavelength is shifted. The change makes the optical output switch between maximum and minimum optical power. The wavelength change is achieved by means of an effective index variation of the ring waveguide, as it can be deduced from Eq. (1.10). Thus, if an effective index varies by an external signal as a consequence of one of the previously mentioned physical methods, a modulated wave is obtained in the way that is shown in Figure 5.

Other important parameters are the cross-coupling coefficient of the light from waveguide to the ring $(k)$, the width of resonance at $-3 \mathrm{~dB}$, also known as width at half maximum (FWHM), and the quality factor, which is a measure of the sharpness of the resonance.

\subsection{Mach-Zehnder Interferometer (MZI) modulator}

The Mach-Zehnder Interferometer is another important modulating structure whose mechanism consists in splitting the input signal into two different arms with integrated phase modulators. If no modulating signal is applied, the signals of both arms are recombined without phase shift difference between them resulting in a constructive interference at the output. 


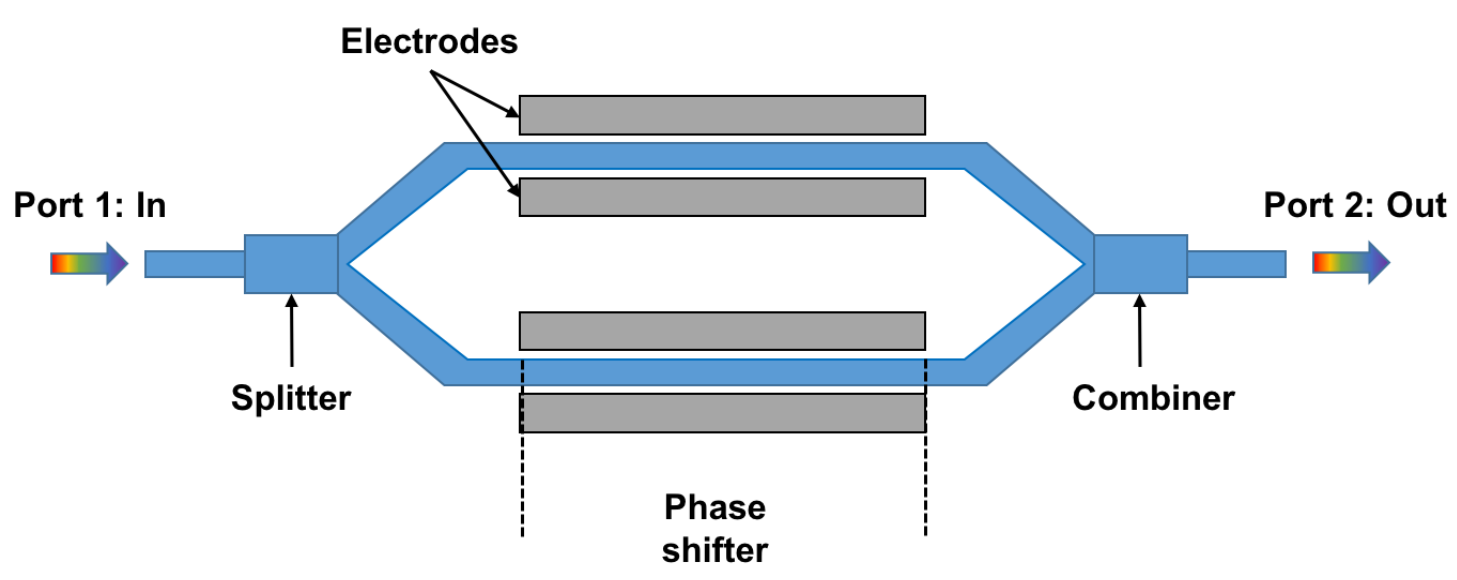

Figure 8. Schematic of a Mach-Zehnder modulator.

However, when a modulating signal is applied to the electrodes, both optical signals are recombined with different phase shift and therefore the output can be converted into an amplitude modulation. The transfer function of the MZI modulator is describing in the following equation:

$$
\frac{P_{o u t}}{P_{\text {in }}}=\cos ^{2}\left(\frac{\Delta \Phi}{2}\right)=\cos ^{2}\left(\frac{\pi}{\lambda_{0}}\left(K_{1}-K_{2}\right) L v(t)\right)
$$

where $K_{1}$ and $K_{2}$ are constants which depends on the physical method used to modify the refractive index and $v(t)$ is the applied voltage or the modulating signal. When the optical phase difference reaches a value of $\pi$ radians, then the interference at the combiner is completely destructive and there is no signal output. The minimum drive voltage to reach such phase shift is called half-wave voltage $\left(V_{\pi}\right)$.

\subsection{Traveling-wave modulator}

The traveling-wave modulator is based on the previously described MZI modulator, but with an important difference: the electrodes design. The electrodes in the MZI modulator (shown in Figure 8) are designed like a capacitor, where two metallic plates are placed along the arms of the MZI modulator. In this way, when a voltage is applied through them an electric field is generated between both metallic plates. For low operational voltages, long arms and therefore long phase shifters are required. As a consequence, high capacitance is obtained and limits the modulator electro-optical bandwidth. Traveling-wave modulators solve this problem by designing the electrodes as a transmission line. When using a 
transmission line as an electrode at high frequencies, the voltage is distributed (not constant) along the electrode, and then the lower capacitance does not limit the electro-optical bandwidth.

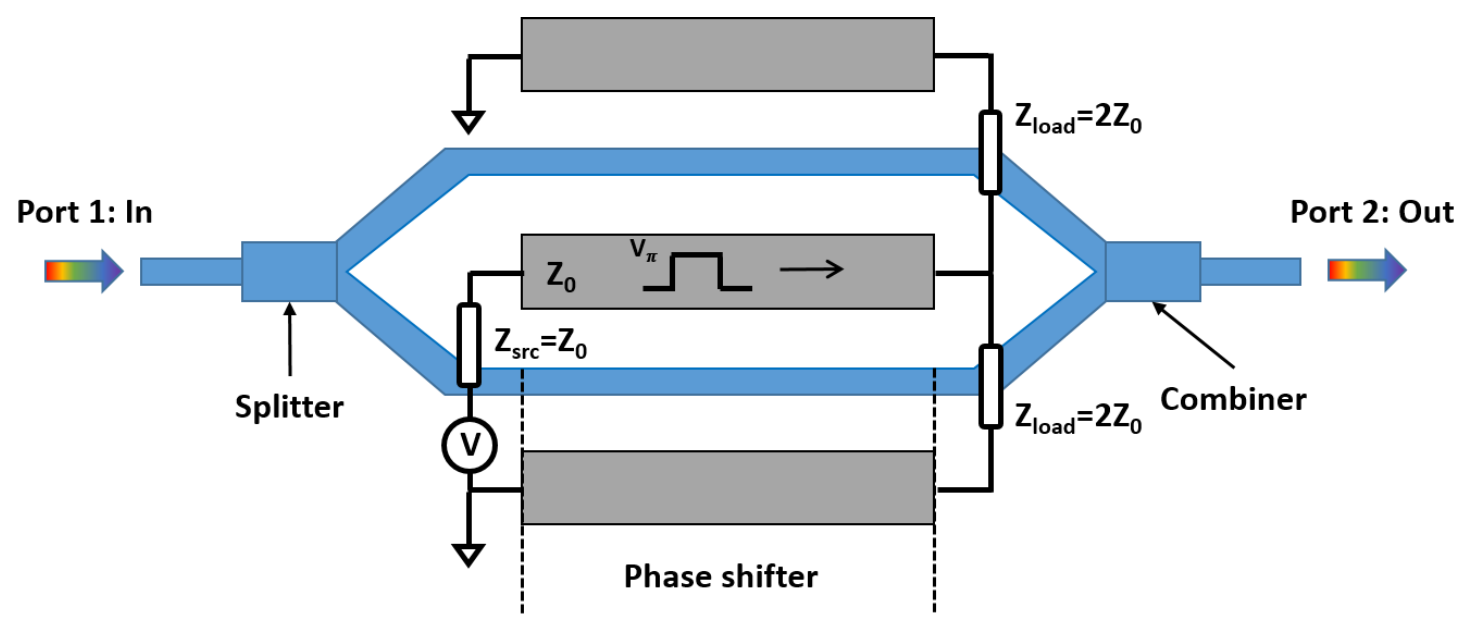

Figure 9. Schematic of a traveling-wave modulator. The electrodes shown are based in a coplanar waveguide design.

Microwave index, transmission line impedance $\left(\mathrm{Z}_{0}\right)$ or propagation losses are crucial parameters that must be taken into account when the electrode is designed to ensure the required performance. Assuming impedance matching $\left(\mathrm{Z}_{\mathrm{src}}=\mathrm{Z}_{0}\right.$, $\mathrm{Z}_{0}=2 \mathrm{Z}_{\text {load }}$ ) of the electrode with the impedance source and load, the next equation describes the traveling-wave modulator electro-optical bandwidth [17], [18].

$$
M(f)=e^{-\left(\frac{\alpha_{m} L}{2}\right)} \sqrt{\frac{\sinh ^{2}\left(\frac{\alpha_{m} L}{2}\right)+\sin ^{2}\left(\frac{\pi f\left(n_{\mu}-n_{o}\right) L}{c_{0}}\right)}{\left(\frac{\alpha_{m} L}{2}\right)^{2}+\left(\frac{\pi f\left(n_{\mu}-n_{o}\right) L}{c_{0}}\right)^{2}}}
$$

where $\mathrm{n}_{0}$ is the optical index, $n_{\mu}$ is the microwave index, $\alpha_{m}$ the propagation losses and $L$ represents the length of the transmission line. Moreover, the electro-optical bandwidth for a lossless transmission line can be extracted from Eq. (1.10) by $M(f)=1 / 2$ and $\alpha_{m}=0$ obtaining the equation (1.11): 


$$
\Delta f=\frac{1.89 c_{0}}{\pi\left(n_{\mu}-n_{o}\right) L}
$$

Thus, taking a look at Eq. (1.11), unlimited bandwidth is theoretically achievable by means of microwave and optical index matching $\left(n_{\mu}=n_{o}\right)$. This theoretical model is useful to understand the role of the microwave index in the design of electro-optical modulators.

\subsection{Objectives and outline of the thesis}

The aim of this thesis has been to improve optoelectronic switches and modulators performance through the study and optimization of the electrical elements. The objectives of this thesis are:

- Development of high efficient micro-heaters for silicon photonic switches.

- Development of slow-wave coplanar waveguides for future application in slow-wave based modulators and switches.

- Development of electrodes for novel modulators and switches based on the integration of barium titanate and vanadium dioxide in silicon devices.

The contents of the thesis are structured in the following chapters:

Chapter 1 spotlights an overview of silicon photonics technology, its implication in the society of the technologies of information and communications, and its evolution and future perspectives. Additionally, a summary has been added with the projects in which this thesis has been involved. Finally, a small review of the main modulation effects and structures has been done to give the essential knowledge of the modulator devices which inspired the work developed in this thesis.

Chapter 2 addresses the design and optimization of optoelectronic switches using micro-heaters. Switching by means of micro-heaters are potentially useful in silicon photonics due to the high silicon thermo-optic coefficient. A multimode interferometer is designed to optimize the thermal response as well as the microheaters. Finally, thermo-optic devices are experimentally characterized in the laboratory. In addition, micro-heaters and its interaction with $\mathrm{VO}_{2}$-silicon based ring switches are also studied and analyzed. 
Chapter 3 delves on the design of novel slow-wave electrodes for application in traveling-wave modulators. This chapter includes a brief introduction of transmission lines theory as well as coplanar waveguides theory and the measurement methods. The microwave index engineering is addressed step by step with the introduction of novel capacitive and inductive elements. Furthermore, the microwave index is obtained by simulations and confirmed through experimental characterization.

Chapter 4 deals with the design of traveling wave electrodes for a MZI modulator for a BTO-Si based modulator. To accomplish the electrode design, the BTO electrical permittivity extraction is the main step. With the BTO electrical permittivity, the electrode and electro-optic design of the modulator has been carried out and, finally, fabricated and experimentally characterized.

Finally, in chapter 5, conclusions and future outline are discussed. 


\section{Chapter 2}

\section{High-efficient micro-heaters design for application in photonic switches}

\subsection{Introduction and State-of-the-Art}

Photonic switches are key elements for inter- or intra-chip routing. Since switching velocity is not as crucial as in modulators, the thermo-optic effect becomes an option to enable switching functionalities. By the appropriate microheater design, the challenge of small size and low power consumption can be accomplished. Next-generation optical networks will have to deal with low power consumption, low latency and high bandwidth requirements in order to exceed the current performance of electrical networks. As a result, silicon photonics is probably the most promising technology to provide these characteristics for short range communications, on chip scale communications and in the future to intrachip communications [19]. Until now, silicon switches have been mostly based on two different photonic structures: ring resonators and Mach-Zehnder interferometric (MZI) structures. Ring resonators allow minimizing the footprint but at expenses of a narrow optical bandwidth which can only be increased by using more complex ring structures or switching techniques [20]-[22]. Furthermore, an additional tuning mechanism, which will increase the total power 
consumption, is also necessary to counteract possible fabrication deviations [23]. On the other hand, silicon MZI switches have a wider optical bandwidth and higher robustness but usually suffer from a larger footprint and higher power consumption that limits the scalability to build switching fabrics with a large number of ports [24]-[31].

\begin{tabular}{|c|c|c|c|c|}
\hline Reference & $\begin{array}{c}\text { Footprint } \\
\left(\mathbf{m m}^{2}\right)\end{array}$ & $\begin{array}{c}\text { Switching } \\
\text { time }(\boldsymbol{\mu s})\end{array}$ & $\begin{array}{c}\text { Power consumption } \\
(\mathbf{m W})\end{array}$ & Structure \\
\hline$[23]$ & 0.0004 & - & 66 & Ring \\
\hline$[24]$ & 0.3 & 30 & 45 & MZI \\
\hline$[25]$ & 0.7 & - & 40 & MZI \\
\hline$[27]$ & 0.05 & 141 & 0.54 & MZI \\
\hline$[28]$ & - & 2.4 & 12.7 & MZI \\
\hline$[29]$ & - & 2.7 & 25 & MZI \\
\hline$[30]$ & - & 10 & 30 & MZI \\
\hline$[36]$ & 2.4 & - & 22 & MMI \\
\hline
\end{tabular}

Table 2. Summary of photonic switches performance based on thermo-optic effect.

New optical switches based on multimode interferometers could provide both small footprint and relaxed fabrication tolerances [32]-[34]. Tunability in MMIs has been reported for different material technologies like InGaAsP [35] and polymers [36]. Table 2 shows the performance of the referenced photonic switches.

In this work, tunability in silicon-based MMIs is demonstrated by means of the thermo-optic effect [37]-[39]. The reduction of power consumption is investigated by engineering the electrical and optical elements of the switching device. In this way, the optimization of the heater geometry is proposed to improve the efficiency of the thermal process [37]. Furthermore, a power consumption reduction of around $50 \%$ is also demonstrated by designing a thermal isolating silica trench within the MMI optical structure [38].

\subsection{The multimode interferometer}

The proposed MMI switch is composed by a multimode silicon waveguide connected to two tapered output and input ports. The tapered ports minimize return losses and provide a wider excitation input and therefore larger self-images into the MMI section. 


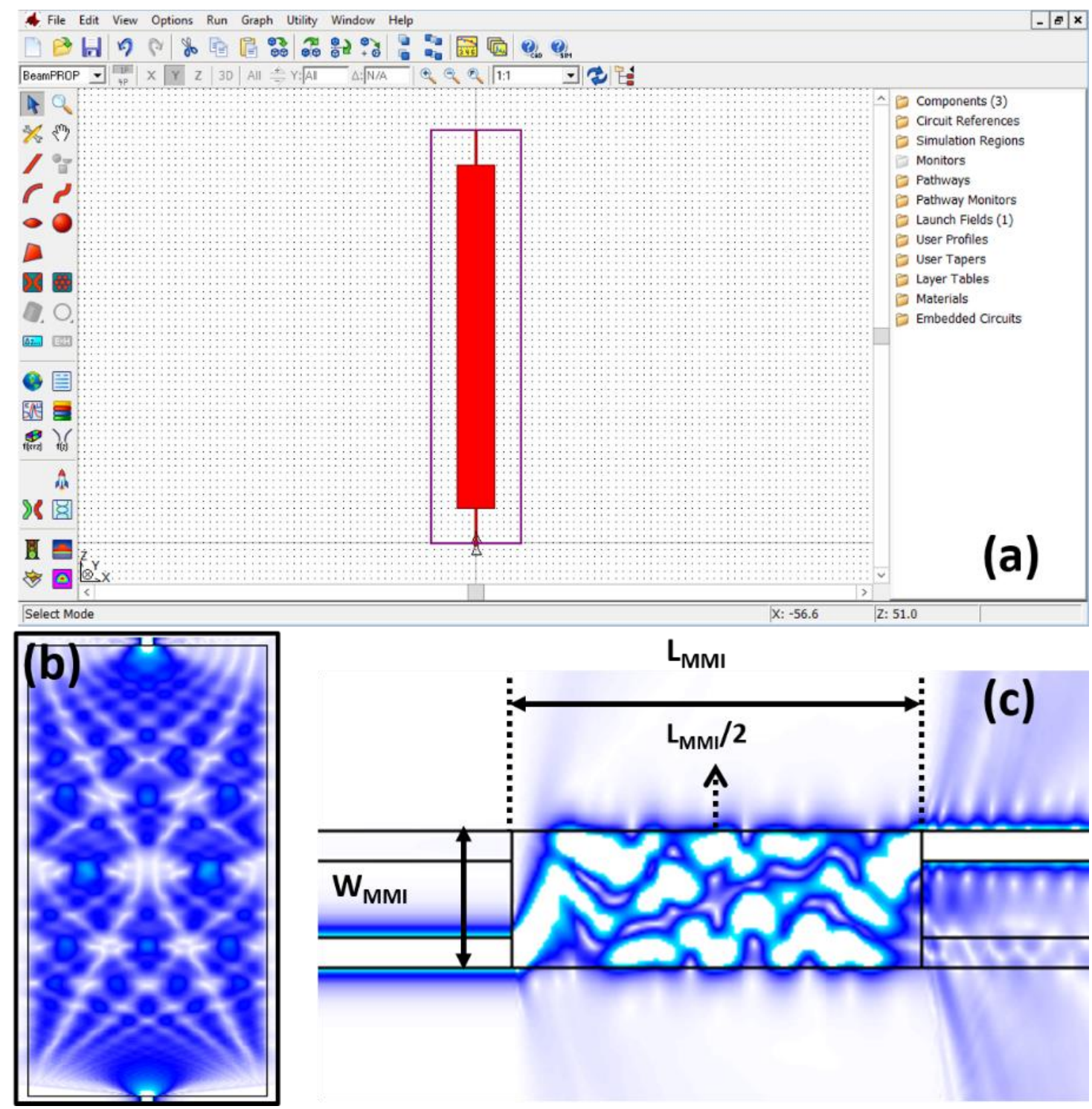

Figure 10. (a)Main interface of RSoft simulation tool. (b) Simulation of a wide 1x1 MMI and, (c) simulation of a reduced size 2x2 MMI using BeamProp method.

Figure 10-a shows the main interface of RSoft simulation design suit with a bigsize 1x1 MMI in order to provide an image which shows the MMI concept (Figure 10-b). RSoft software is used to design and analyze optical telecommunication devices, optical components used in semiconductor manufacturing and nano-scale optical structures. RSoft also enables engineers to design and optimize optical components and systems. The main used tool for MMI simulation provided by Rsoft is the BeamPROP which is based on the numerical method known as beam propagation method (BPM). Additionally, Figure 10-c shows a 2x2 MMI simulation which will be taken as a reference for the design of $2 \times 2$ tunable MMI. 


\subsection{Tunable multimode interferometer design}

The tunable MMI parameters have been designed taking into account the tapered ports. A MMI length ( $\left.\mathrm{L}_{\mathrm{MMI}}\right)$ of $594.5 \mu \mathrm{m}$ and MMI width $\left(\mathrm{W}_{\mathrm{MMI}}\right)$ of $9 \mu \mathrm{m}$ have been obtained thus giving rise to a compact footprint of around $0.005 \mathrm{~mm}^{2}$. The switching functionality is achieved by inducing a $\pi$-phase shift of one of the two self-images located under the electrodes at $\mathrm{L}_{\mathrm{MMI}} / 2$ (see sketch in Figure 11-a). This phase shift results in a new mirrored interference pattern that propagates after the electrode [34].

Additionally, due to the high losses generated by the metal and optical mode interaction, the gap between the electrode and the heater should be carefully studied. The inset of Figure 11-b shows the silicon oxide gap which separates the MMI and the electrode. Furthermore, the graphic of Figure 11-b describes the insertion losses of the MMI as a function of the gap. In this case, in order to limit the losses and with the aim of minimizing the fabrication deviations, a gap value of $0.7 \mathrm{\mu m}$ has been selected. For such gap value, insertion losses as low as 0.97 $\mathrm{dB}$ are achieved.
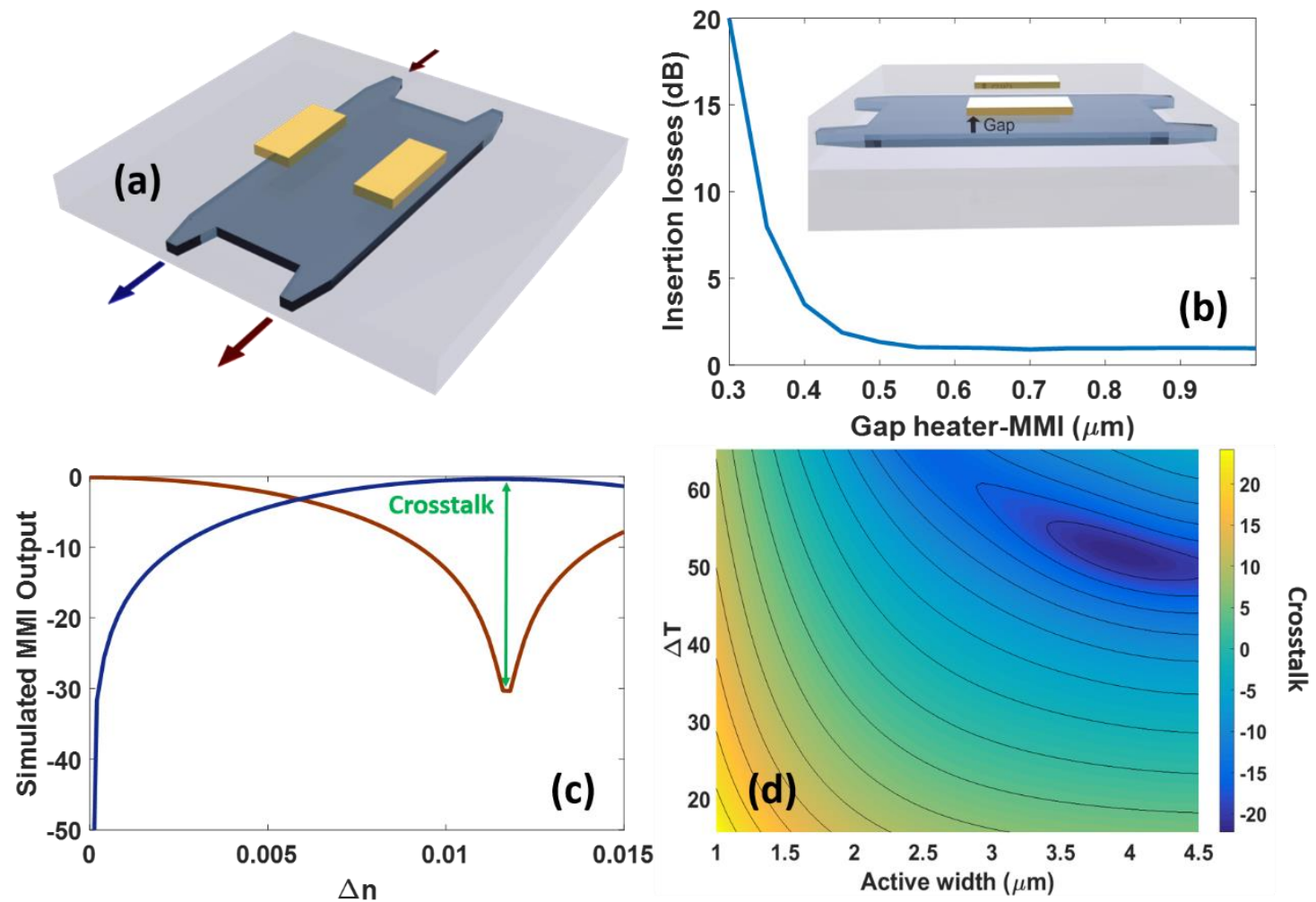

Figure 11. (a) Sketch of the tunable MMI, (b) sketch of MMI with the electrode gap and insertion losses as a function of the MMI-heater gap (c) Transmission response as a function of the index variation. (d) Crosstalk dependence on the width of the active area where the silicon index is changed. 
The relationship between phase and index change can be calculated as:

$$
\Delta \phi=k \Delta n L_{h}
$$

where $L_{h}$ is the heater length to achieve the required phase shift and $k=2 \Pi / \lambda$ is the wavelength number. The index change $(\Delta n)$ is determined by the thermal coefficient $(\partial n / \partial T)$ of the material. Therefore, it can be calculated as:

$$
\Delta n=\frac{\partial n}{\partial T} \Delta T
$$

where the thermal coefficient for silicon is $1.84 \cdot 10^{-4} \mathrm{~K}^{-1}$. Thereby, a compact heater of $80 \mu \mathrm{m}$ length can be used to reach a $\Pi$-phase shift if the index change is over $\Delta n \approx 10^{-2}$, which would be obtained with a $\Delta T \approx 55 \mathrm{~K}$ between both selfimages.
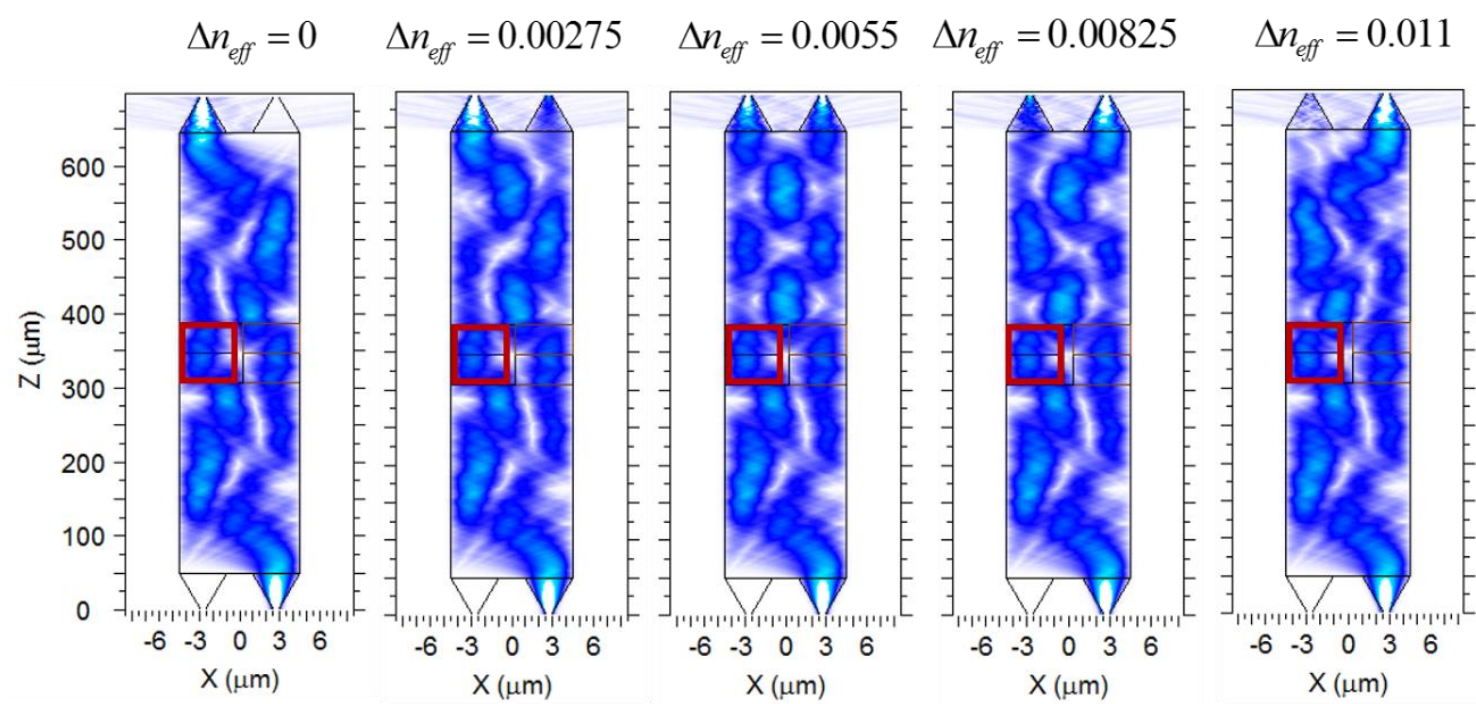

Figure 12. Interference pattern of MMI gradually tuned as a function of the effective index variation. The red square highlights the index variation area.

Once the heater length has been chosen, the optimum width has been designed through 3D simulations carried out with, RSoft. Thus, the transmission response as a function of the index variation is shown in Figure 11-c. Furthermore, Figure 11-d shows the simulated for different temperatures and as a function of the active width results, where it can be seen that a heater width of $3.7 \mu \mathrm{m}$ is required to maximize the crosstalk over $20 \mathrm{~dB}$. Additionally, to understand what is happening 
inside the tunable MMI, Figure 12 shows different images where the refractive index is gradually tuned in the active region, and finally, the output is switched form the cross-port to the bar-port.

\subsection{Thermal optimization of the multimode interferometer}

Thermal simulations have also been performed using Comsol Multiphysics ${ }^{\circledR}$ with a 3D finite element method (FEM) to analyze the thermal crosstalk of the MMI structure and how it does affects the resultant phase shift. Comsol Multiphysics ${ }^{\circledR}$ provides the appropriate software tools which allows to run thermal simulations and analyze the obtained results by means of Joule heating effect calculations. The Joule heating effect is described by conservation laws for electric current and energy. Once solved for, the two conservation laws give the temperature and electric field, respectively. All surfaces, except the bolt contact surfaces, are cooled by natural convection in the air surrounding the structure. Furthermore, Joule heating module contains simulation tools to study the mechanisms of heat transfer in collaboration with other physics, such as structural mechanics, fluid dynamics, electromagnets, and chemical reactions. Such simulation can be applied to any project, process, or product.

Figure 13 shows the main interface of the simulation software Comsol Multiphysics $^{\circledR}$. The left panel is called Model Builder and organize the main simulation characteristics. Device geometry, used materials, applied physics, study, mesh and results analysis can be controlled or modified in the Model Builder. Furthermore, the top bar is set for adding new and required functions over or to the Model Builder if it is necessary at some time. Additionally, Figure 13 shows how it is possible to go from a $3 \mathrm{D}$ simulation and obtain a $2 \mathrm{D}$ profile image of the resulting simulation. 

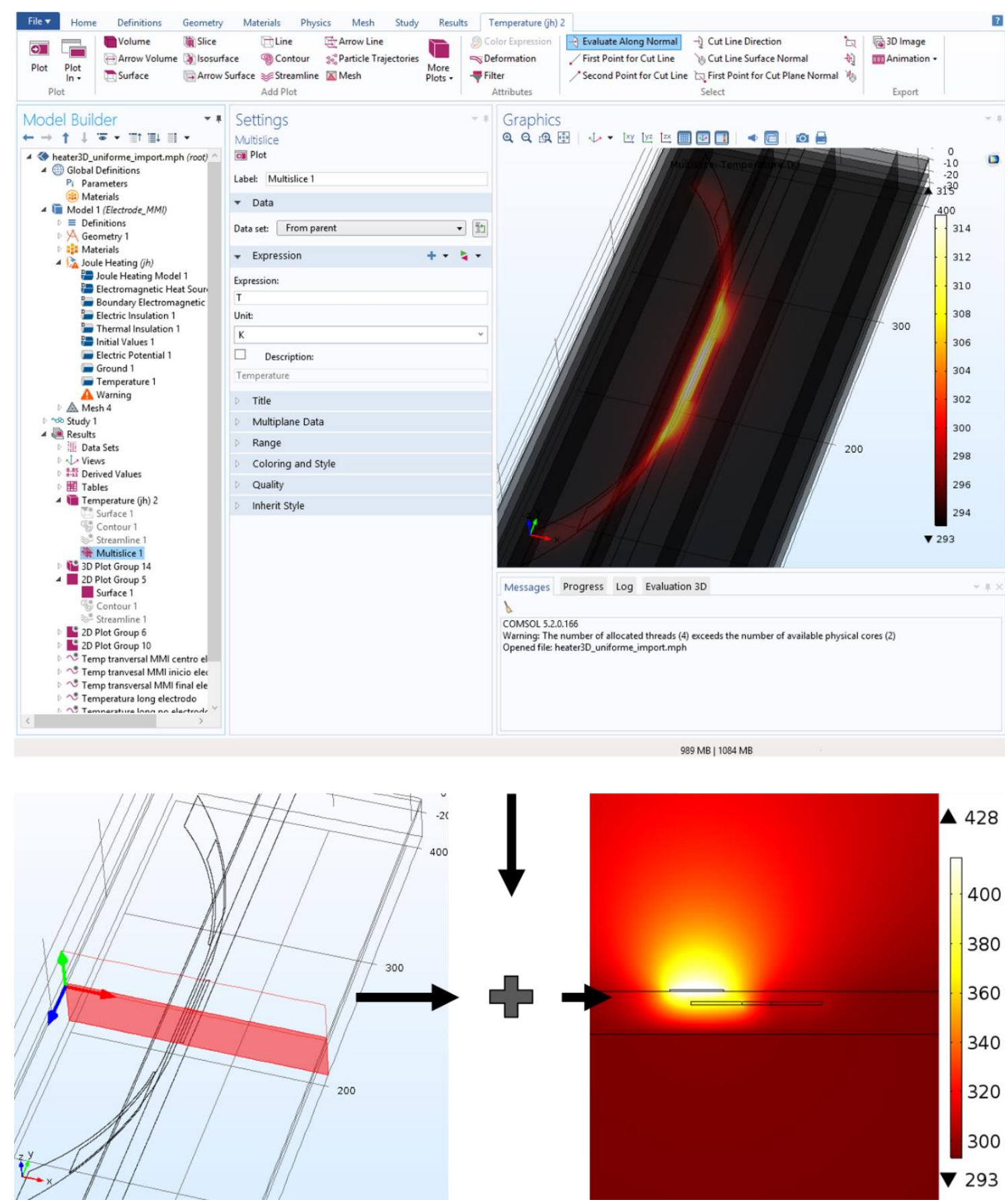

Figure 13. Comsol Multiphysics main interface, and 2D plain obtained from a 3D simulation.

Regarding to the tunable MMI design and optimization, the heater was located on top of a $700 \mathrm{~nm}$ thick silica upper cladding to avoid optical losses and just on the active region over the self-image on the MMI (see Figure 11-b, or Figure 12). However, a high thermal crosstalk has been shown as an undesirable effect due to the high silicon thermal conductivity of $149 \mathrm{~W} /(\mathrm{mK})$ [40]. Therefore, a power consumption of $60.4 \mathrm{~mW}$ has been required to reach a $\Delta \mathrm{T} \approx 55 \mathrm{~K}$ between both self-images at $\mathrm{L}_{\mathrm{MMI}} / 2$. In Figure 14-a the high thermal crosstalk can be observed. 

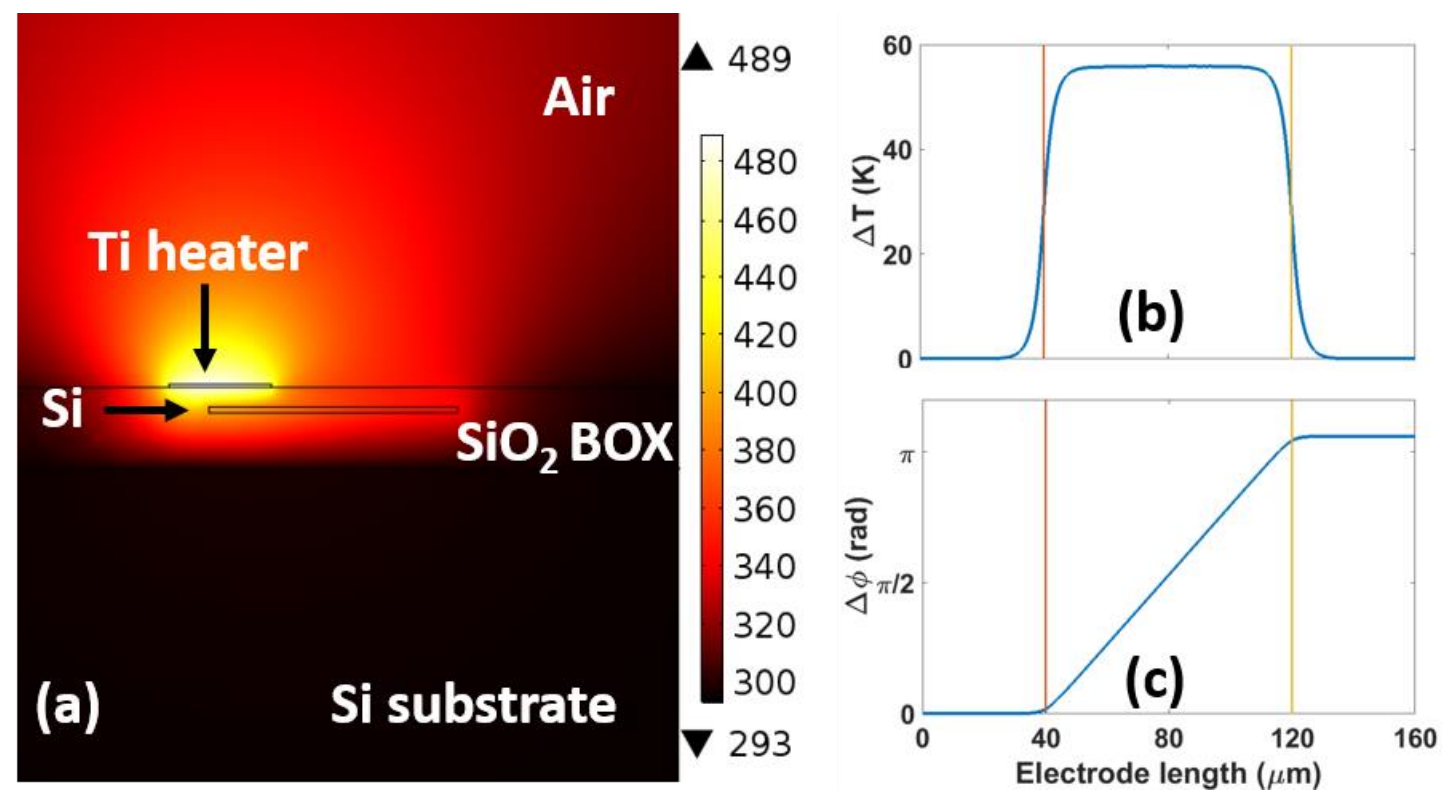

Figure 14. (a) Heat distribution in a transversal cut of the MMI structure, (b) temperature difference between both sides of the MMI along the propagation direction and (c) accumulated phase shift obtained from the temperature difference. Results have been obtained for an applied electrical power of $60.4 \mathrm{~mW}$.

Furthermore, Figure 14-b shows the temperature difference between both sides of the MMI structure along the propagation direction. Vertical left and right lines (red and yellow) represents the beginning and the end of the heater. Additionally, Figure 14-c depicts the accumulated phase shift along the MMI self-image length taking into account the temperature difference reported in Figure 14-b. It can be seen that the reached phase shift is above $\Pi$ radians with an applied power consumption of $60.4 \mathrm{~mW}$. However, it is still a high value for low power applications and thus a solution to minimize the power consumption has been investigated.

To decrease the power consumption, it is required to achieve a thermal isolation between the self-images, which allows to reduce the thermal crosstalk. A silica trench has therefore been etched in the middle of the silicon MMI structure. The optimum location to minimize the impact on the MMI performance in terms of insertion loss is found to lie where the optical field is weak enough, that is, around $350 \mu \mathrm{m}$ with respect to the z-axis as can be seen in Figure 12 and Figure 15-a.

Once the best position has been selected, the optimum length and width of the silica trench has been designed. A tradeoff between minimizing the insertion losses and achieving a large thermal isolation has been found. The optimum size of the trench has been obtained for a trench length and width of $50 \mu \mathrm{m}$ and $2 \mu \mathrm{m}$, respectively, giving rise to an insertion loss of around $2 \mathrm{~dB}$ (see Figure 15-b). Further optimization of the MMI parameters was carried out so that the insertion losses were reduced from 2 to $0.5 \mathrm{~dB}$ by increasing the MMI length up to $616 \mu \mathrm{m}$. 
Figure 15-c shows the optical field distribution of the optimized MMI structure with the silica trench. Despite the fact that the interference pattern is slightly distorted due to the trench presence, it remains without significant alterations.

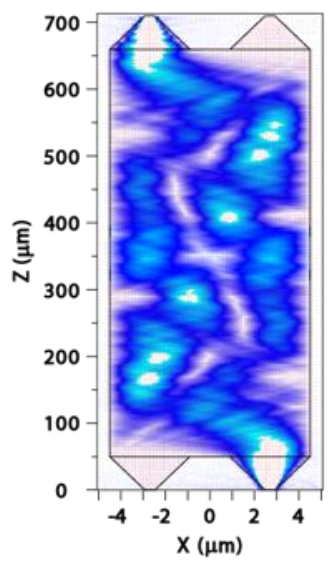

(a)

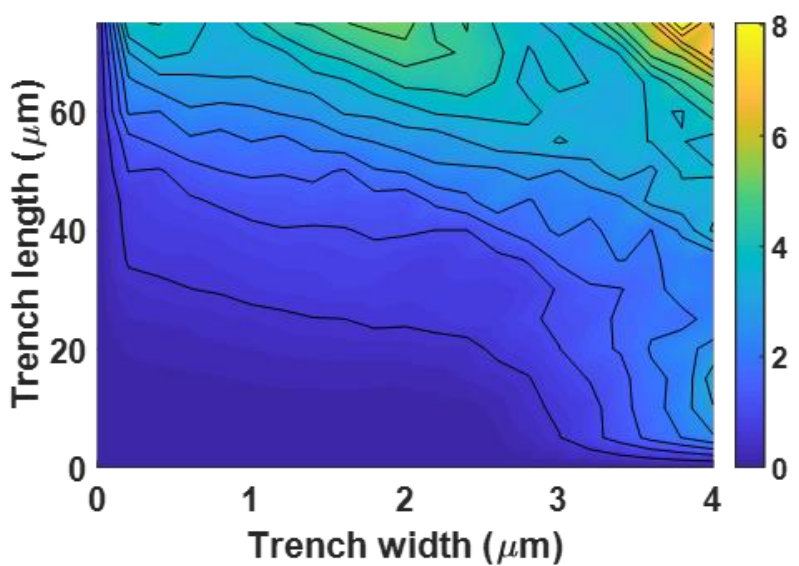

(b)

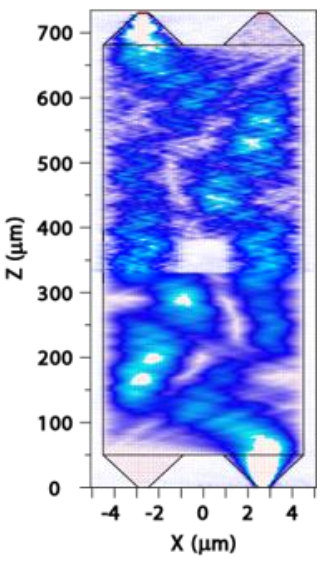

(c)

Figure 15. (a) Optical field distribution in the conventional MMI structure. (b) Insertion losses versus silica trench dimensions. (c) Optical field distribution in the MMI structure with optimized silica trench.

A comparison through thermal simulation between both proposed MMI structures in Figure 15-a and 15-c has been carried out in order to evaluate its usefulness. As can be seen in Figure 16-a and 16-b, the heat generated is more concentrated in the area of interest, and as a consequence the top heat reached is higher in the structure with the silica trench. In addition, Figure 16-c shows the temperature increase as a function of the power consumption for both analyzed structures, and it can be clearly seen that the structure with the silica trench reaches higher temperatures. This makes the structures with the silica trench more efficient that the common MMI structure.

Figure 17-a shows the heat distribution in the optimized MMI structure for the same transversal cut than in Figure 14-a. It can be clearly seen that the heat is concentrated on the heater side owing to the isolation gap unlike in the conventional MMI structure in which the heat spreads out along the silicon layer. The $\Pi$ radians phase shift was reached (Figure 17-c) in the optimized MMI structure with a power consumption of $35.4 \mathrm{~mW}$, which means a reduction of approximately $45 \%$ compared to the conventional MMI structure without trench. 

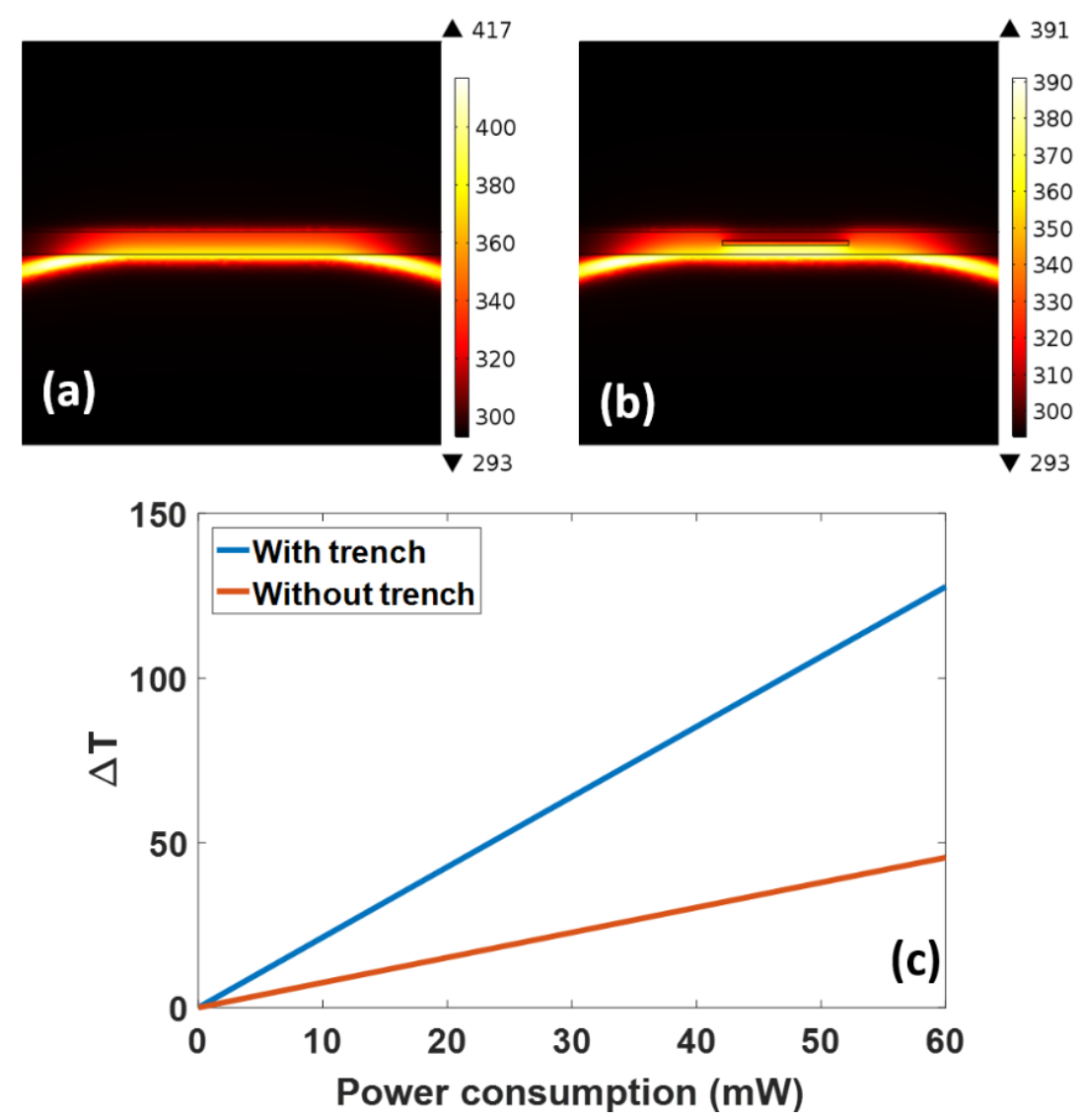

Figure 16. Top view of the MMI section where the heater is placed (a) without silica trench and (b) with silica trench. (c) Comparison of the $\Delta \mathrm{T}$ increase between both proposed structures.
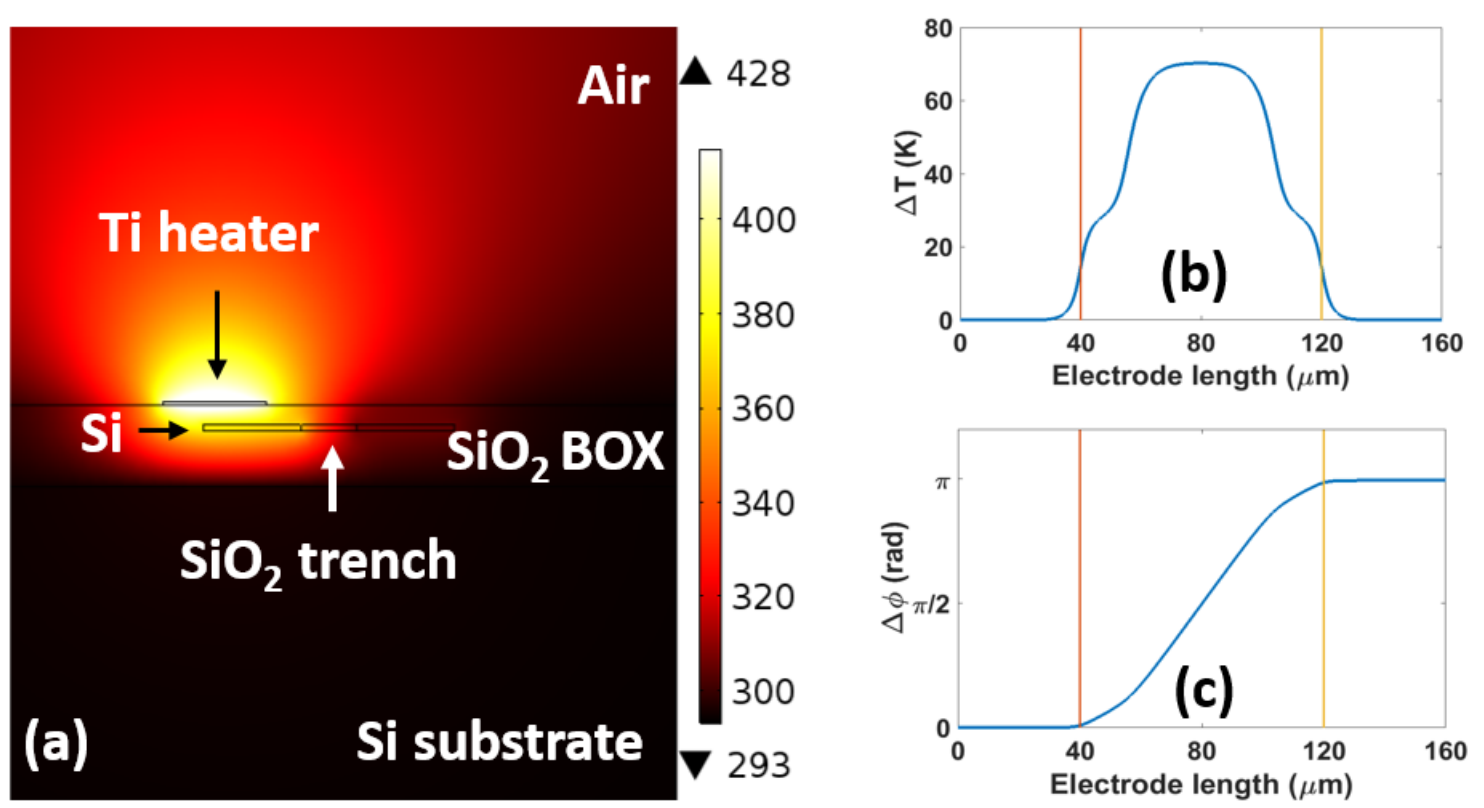

Figure 17. (a) Heat distribution in a transversal cut of the MMI structure with silica trench, (b) longitudinal temperature difference between both sides of the MMI and (c) accumulated phase shift obtained from the temperature difference. Results have been obtained for an applied electrical power of $35.4 \mathrm{~mW}$. 


\subsection{Micro-heater design for multimode interferometers}

The switching performance can be further enhanced by optimizing the electrodes with the aim of improving the power consumption. Therefore, we have optimized both the heater and the electrodes. The difference between the electrode and the heater should be pointed out: the heater is the metal section designed to achieve a highly localized temperature in the MMI structure (see Figure 17-a), and the electrode includes the whole electrical circuit consisting of the pads and the heater. Therefore, the electrodes can be defined as a combination of three resistances: two resistances for each one of the pads and the last one for the heater. A schematic of the electrode is shown in Figure 18. To improve the performance of the electrode, the heater should dissipate the maximum amount of the total electrical power.

\section{Electrode}

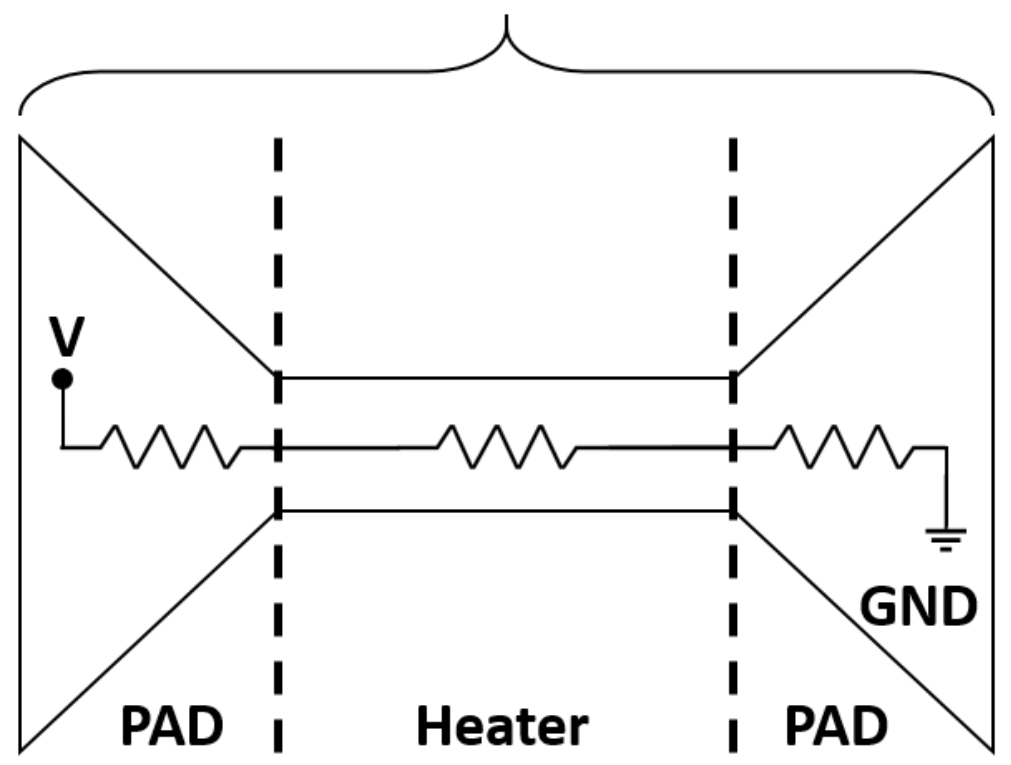

Figure 18. Schematic electrode for thermo-optic effect based switches.

In order to analyze the electrode behaviour, and with the aim of minimizing the required power consumption, a simple electrical modelling can be carried out. Let start with the following equations:

$$
\begin{aligned}
& P_{H}=I^{2} R_{H} \\
& P_{P}=I^{2} R_{P}
\end{aligned}
$$


where $P_{P}$ and $P_{H}$ is the dissipated power by the pad and the heater respectively. $R_{H}$ is the heater resistance and $R_{P}$ is the pad resistance. The current flowing through the electrode is represented by $I$. It is possible to obtain the circuit current from equation (2.3) and (2.4):

$$
\begin{gathered}
I=\sqrt{\frac{P_{H}}{R_{H}}} \\
I=\sqrt{\frac{P_{P}}{R_{P}}}
\end{gathered}
$$

the total power consumption is expressed by the following equation:

$$
P_{T}=I^{2} R_{T}=I^{2}\left(2 R_{P}+R_{H}\right)
$$

where $P_{T}$ and $R_{T}$ are the total power consumption and the total resistance respectively. To optimize the electrode power consumption, it must be analyzed as a function of the heater power consumption and the circuit resistance. Therefore, by replacing equation (2.5) in (2.7), the following equation (2.8) is obtained:

$$
P_{T}=\frac{P_{H}}{R_{H}}\left(2 R_{P}+R_{H}\right)=P_{H}\left(\frac{2 R_{P}+R_{H}}{R_{H}}\right)=P_{H}\left(1+2 \frac{R_{P}}{R_{H}}\right)
$$

To obtain a highly efficient electrode, the maximum power consumption should be concentrated in the heater. Thus, equation (2.8) describes the total power consumption as a function of the heater power consumption, the pad resistance and the heater resistance. Assuming the pad resistance as an undesirable parameter to optimize the electrode which is directly related with the whole electrode resistance, the next solutions, for equation (2.8), can be found: 


$$
\begin{aligned}
\lim _{R_{P} \rightarrow R_{H}} P_{T} & =3 P_{H} \\
\lim _{R_{P} \rightarrow 0} P_{T} & =P_{H}
\end{aligned}
$$

As it can be deduced from equation (2.9), if the pad and the heater have a similar resistance, the total power consumption is 3 times the power consumed by the heater. The power consumption is the same in the pad as in the heater, which means that $2 / 3$ of the total power consumption is useless. However, if the pad resistance becomes negligible the total power consumption also becomes the heater power consumption and therefore the total power consumption is used for tuning the MMI response.

In such way, two different and compatible solutions are proposed in order to design highly efficient micro-heaters. The first one is focused on the pads resistivity issue. This solution addresses the use of different materials for the pads and the heater fabrication. Using a high-resistive material in the heater and highconductive material for the pads, the power consumption is focused on the heater. The chosen materials are titanium $\left(2.38 \times 10^{6} \mathrm{~S} / \mathrm{m}\right)$ for the heater and cooper $\left(5.81 \times 10^{7} \mathrm{~S} / \mathrm{m}\right)$ for the pad. The second solution is focused on the power consumption optimization of the heater by means of its shape. Straight heater (Figure 19-a) is compared with a meander heater (Figure 19-b) in order to study its differences and choose the best solution. Meanwhile, Figure 19-c shows the whole structure with electrodes, MMI and the input and output ports.
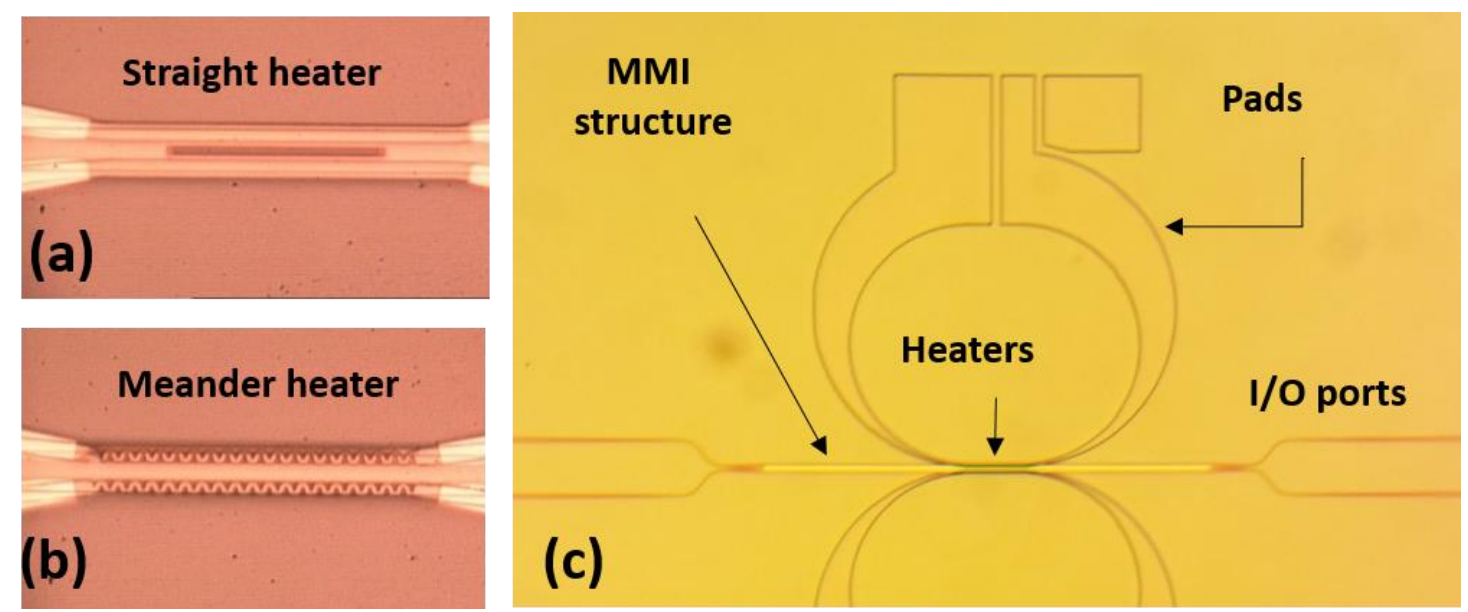

Figure 19. (a) Straight heater in the MMI with the silica trench, (b) meander heater in the MMI without silica trench and (c) $2 \times 2$ switch with electrodes consisting of the copper pads and the titanium heaters on top of the MMI structure. In (a) and (b) the double metallization is observed. 
Thermal simulations have been previously carried out to determine the different behavior between the straight and the meander heaters as well as the different power consumption achieved by both proposed heaters for tuning the MMI response.

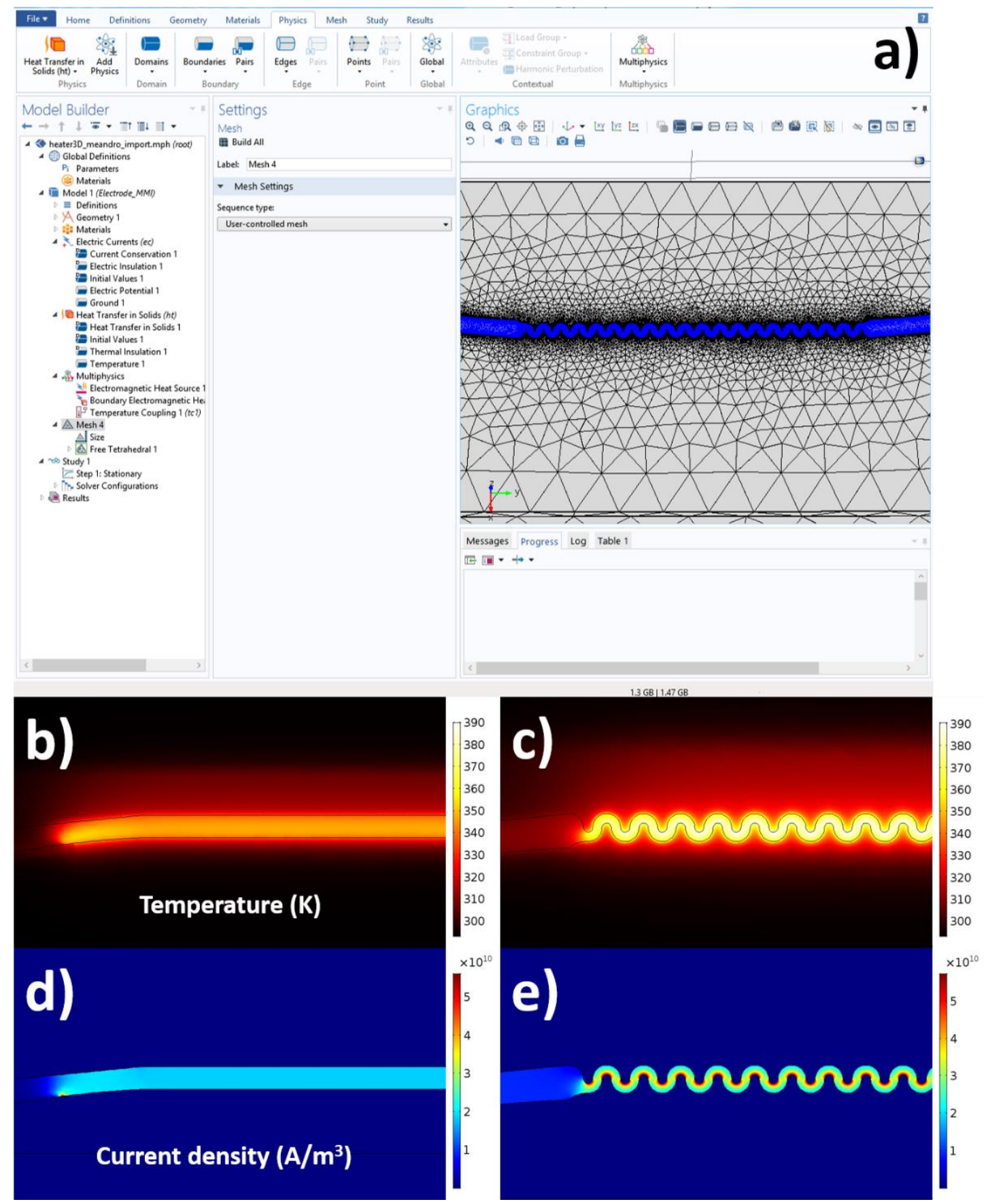

Figure 20. (a) Comsol user interface. (b) and (c), for the same power consumption, the temperature reached for both heaters, straight and meander respectively. (d) and (e) current density for both heaters.

Figure 20-a shows the main Comsol interface with a 3D mesh of the meander heater over the silica cladding. Regarding to the heater study, Figures 20-b and 20-c shows the temperature reached by both heaters, straight and meander 
respectively. In this way, for the same power consumption in both simulation, the final temperature reached for the meander is up to $390 \mathrm{~K}$ in comparison with $345 \mathrm{~K}$ obtained by the straight heater. Such difference is fundamentally due to the width difference between the heaters. With a meander shape, the heater cover-up the self-image of the MMI in the same way that straight heater does.

Additionally, Figures 20-d and 20-e show the current density for straight and meander heaters respectively. Paying attention to Figure 20-e, one can see how the maximum amount of current is concentrated on the inside of the meander corner. Such effect is due to the fact that the electrical current always follows the minimum resistance path, and as a result, observing carefully on the meander heater, it is possible to realize that the heat generated on the corners inside is about $10 \mathrm{~K}$ greater than on the outside. Thus, that small effect also benefits to reach the maximum temperature and minimize the power consumption.

Finally, thermal simulations as shown in Figures 14 and 17 have also been carried out to determine the power consumption reduction achieved with the proposed electrodes. Table 3 summarizes the obtained simulation results. It can be seen that a power consumption reduction of around $25 \%$ has been achieved for the meander heater.

\begin{tabular}{|c|c|c|}
\hline MMI structure & Straight heater & Meander heater \\
\hline Without silica trench & $60.4 \mathrm{~mW}$ & $53.32 \mathrm{~mW}$ \\
\hline With silica trench & $35.4 \mathrm{~mW}$ & $26.5 \mathrm{~mW}$ \\
\hline
\end{tabular}

Table 3. Simulated power consumption for different heater and MMI configurations.

\subsection{Device fabrication}

The optical switches based on the proposed MMI structures and electrodes were fabricated by using electron-beam lithography, inductive coupled plasma (ICP) etching and metal evaporation in the Valencia Nanophotonics Technology Center (www.ntc.upv.es). The structures were fabricated on standard SOI samples from SOITEC wafers with a top silicon layer thickness of $220 \mathrm{~nm}$ and a buried oxide layer thickness of $2 \mu \mathrm{m}$. The fabrication is based on an electron beam direct writing process performed on a coated $100 \mathrm{~nm}$ hydrogen silsesquioxane resist film. The mentioned e-beam exposure was optimized in order to reach the required dimensions employing an acceleration voltage of $30 \mathrm{KeV}$ and an aperture size of $30 \mu \mathrm{m}$. 

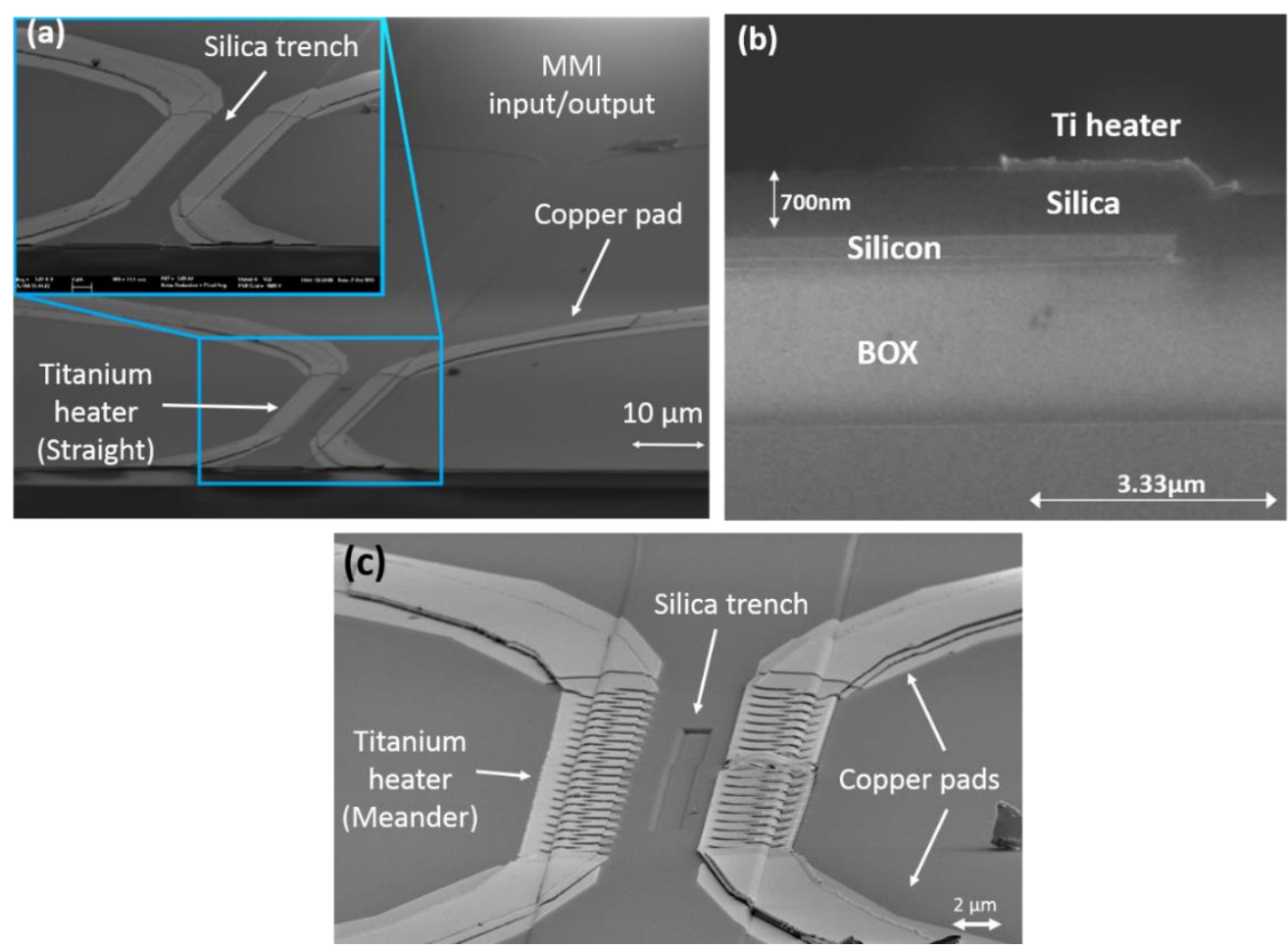

Figure 21. (a) SEM image of the 2x2 MMI switch with the straight electrodes. (b) Transversal SEM image of the MMI structure with the titanium heater. (c) Another SEM image of the 2x2 MMI switch, in this case, with the meander electrodes. The silica trench can be shown in (a) and (c).

After developing the hydrogen silsesquioxane resist using tetramethylammonium hydroxide as developer, the resist patterns were transferred into the SOI samples employing an also optimized ICP reactive ion etching process with fluoride gases. A $700 \mathrm{~nm}$ thickness silicon dioxide upper cladding was deposited on the SOI sample by using a plasma enhanced chemical vapour deposition (PECVD). Finally, the electrodes fabrication was implemented by two e-beam positive resist exposure (PMMA) prior to a metal evaporation and lift-off processes. In the first exposure, $100 \mathrm{~nm}$ of titanium was deposited on the MMI areas while in the second exposure process the $100 \mathrm{~nm}$ copper pads were exposed. Figures 21-a, 21-b and 21-c show scanning-electron microscope (SEM) images of the fabricated devices.

\subsection{Characterization and experimental results}

The fabricated devices have been characterized and their performance compared step by step. The schematic set up consists in a tunable CW laser source, a polarization controller which goes to the device under test (DUT), and the optical output to the power meter. The DC power supply is connected to an ampere meter, and then, to the electrical paths in the DUT. 
Finally, a CW laser source and the power meter are controlled by a computer software based on Lab-View. A schematic set-up is shown in Figure 22, and additionally, Figure 23 shows pictures of the measurement set-up.

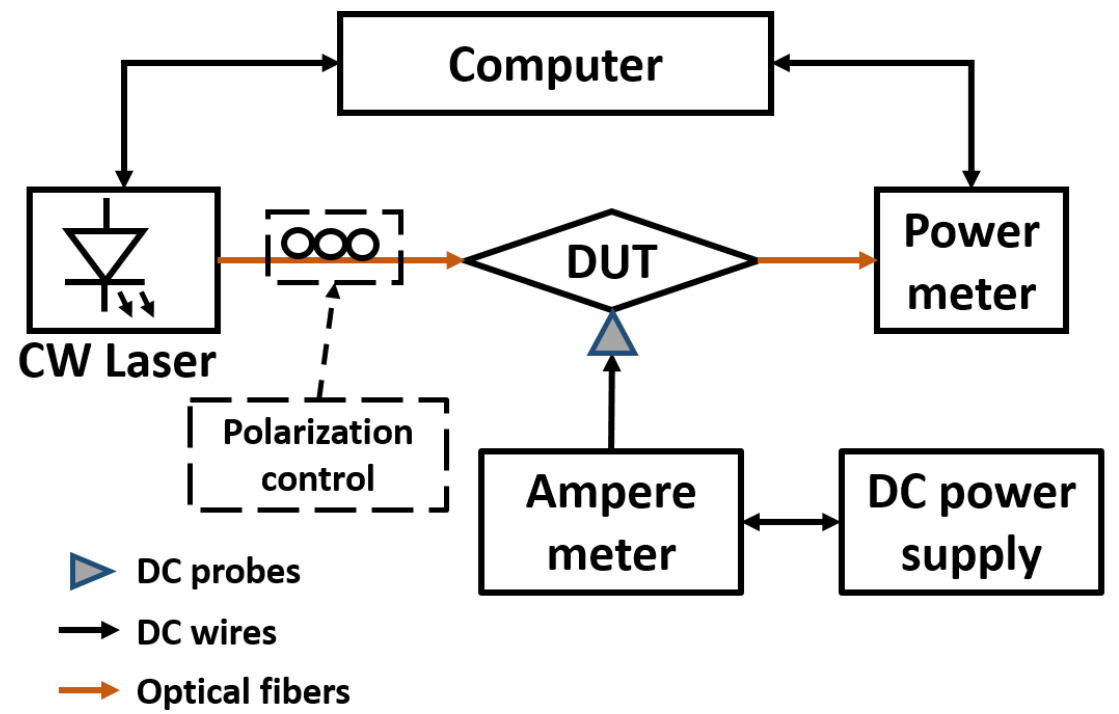

Figure 22. Schematic of measurement set-up to characterize the tunability of the designed MMIs and the power consumption.

Figure 24-a shows the optical power at both output ports as a function of the applied electrical power for the switches based on the MMI with and without the silica trench. In this case, titanium electrodes have been used in both MMI switches. As it can be observed, the required electrical power to switch between the bar and cross port is reduced by a factor of two approximately, from 254.2 $\mathrm{mW}$ to $122.7 \mathrm{~mW}$.

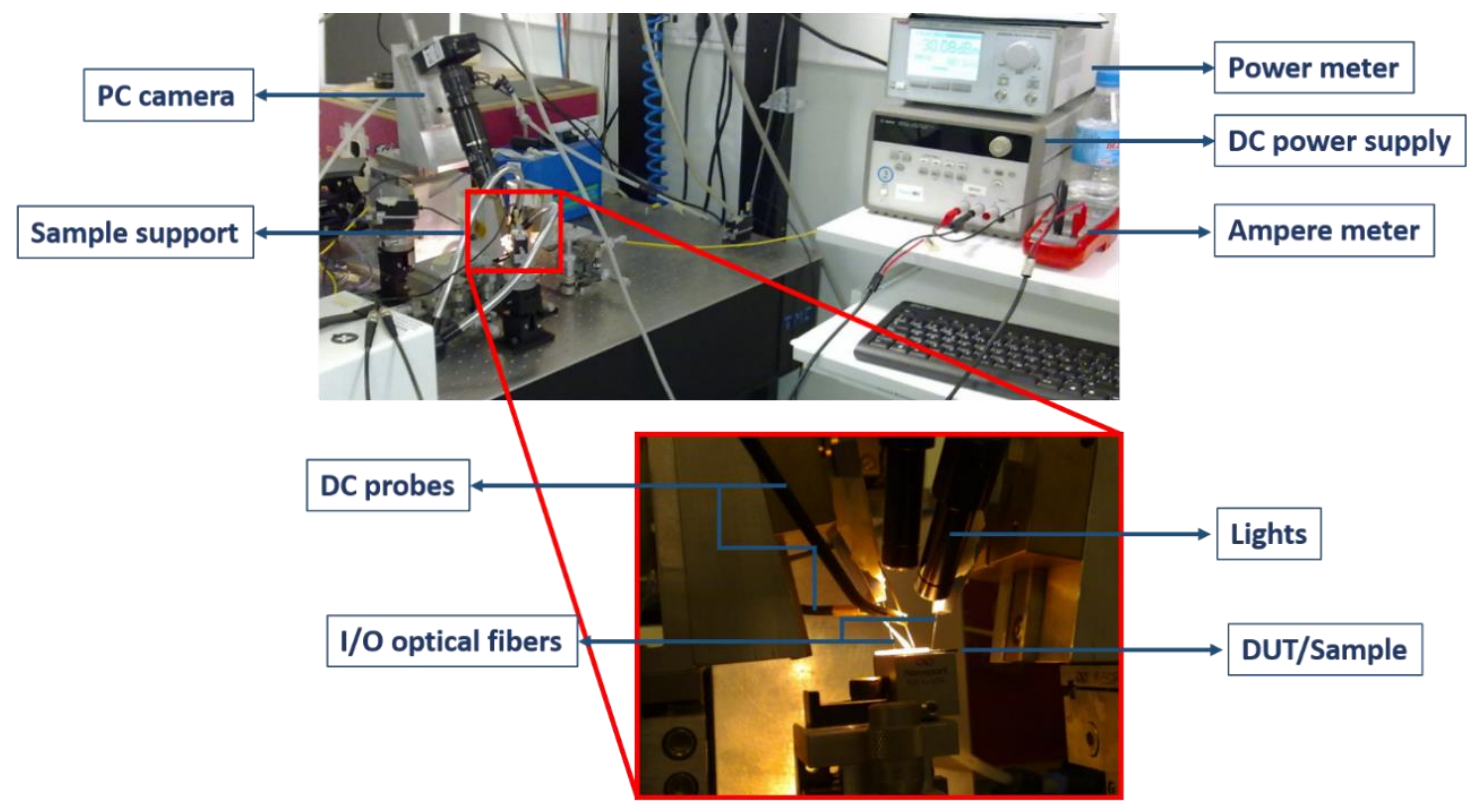

Figure 23. Pictures of the measurements set-up to characterize the tunability of previously designed MMIs and the power consumption. 

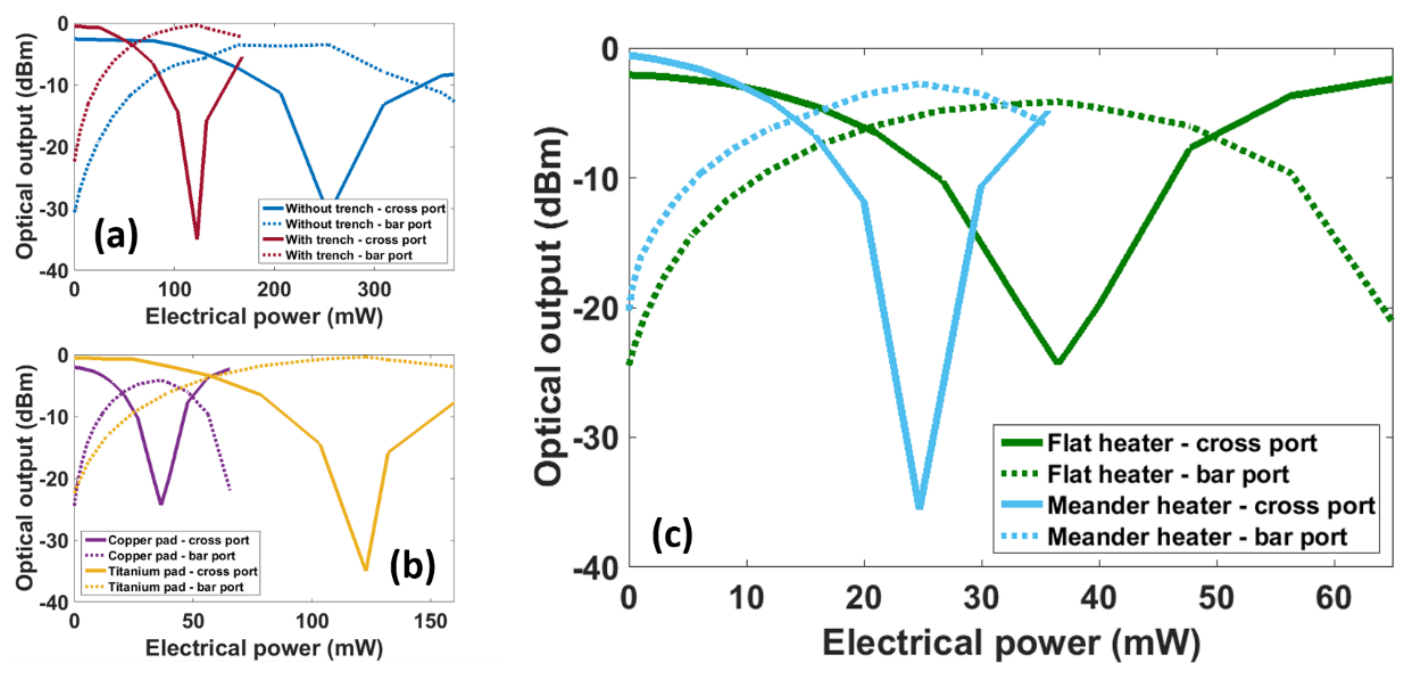

Figure 24. Switching response of the MMI structure (a) with silica trench and without silica trench by using titanium pads, (b) with silica trench and electrodes with copper and titanium pads, (c) with silica trench and with the straight and meander heaters, both with cooper pads.

Once this improvement has been demonstrated, the MMI structure with silica trench has been used to obtain the next results. Figure 24-b shows the comparison between different electrodes based on copper and titanium pads. In this case, there is a significant power consumption reduction, from 122.7 to $36.5 \mathrm{~mW}$, due to the huge amount of electrical power wasted when using titanium pads compared with the electrode with copper pads. The introduction of copper pads means a reduction close to $70 \%$ which is very consistent with the simulation and the theoretical development of the section 2.5. Therefore, copper pads were considered for the final structures. Figure 24-c shows the switching response for the different designed heaters: straight and meander. It can be seen that the power consumption is $36.5 \mathrm{~mW}$ for the straight heater and $24.9 \mathrm{~mW}$ for the meander heater. Therefore, a reduction of around 30\% has been achieved in power consumption. Taking into account the initial power consumption of $245.2 \mathrm{~mW}$ and the final value of $24.9 \mathrm{~mW}$, a $90 \%$ reduction has been demonstrated. Furthermore, there is an excellent agreement with the simulation results for both power consumption and optical performance as the small impact on the insertion losses and crosstalk.

In addition to power consumption, another important feature in optical switches is the time response. Therefore, the switching time of each structure has also been measured and results are shown in Table 4. In the cross port, the fall time is associated with the heating process while the rise time is associated with the cooling process. The opposite behavior occurs in the bar port. Clearly the cooling process is the limiting factor in the switching speed. Furthermore, it can be seen that the time response of the MMI structure with silica trench during the cooling 
process is around four times slower than in the MMI structure without trench. Such behavior is attributed to the small amount of heat that is transferred to the environment due to the thermal isolation provided by the silica trench. On the other hand, it can also be observed that the heater geometry has a low dependence upon the switching time although slightly lower cooling times have been achieved for the meander heater. As a result, the meander heater is the best option as power consumption is also minimized, as depicted in Figure 24-c. Regarding to the switching time measurements, Figure 25 shows the set-up schematic, and Figure 26 shows some images of the laboratory with the ongoing measurements.

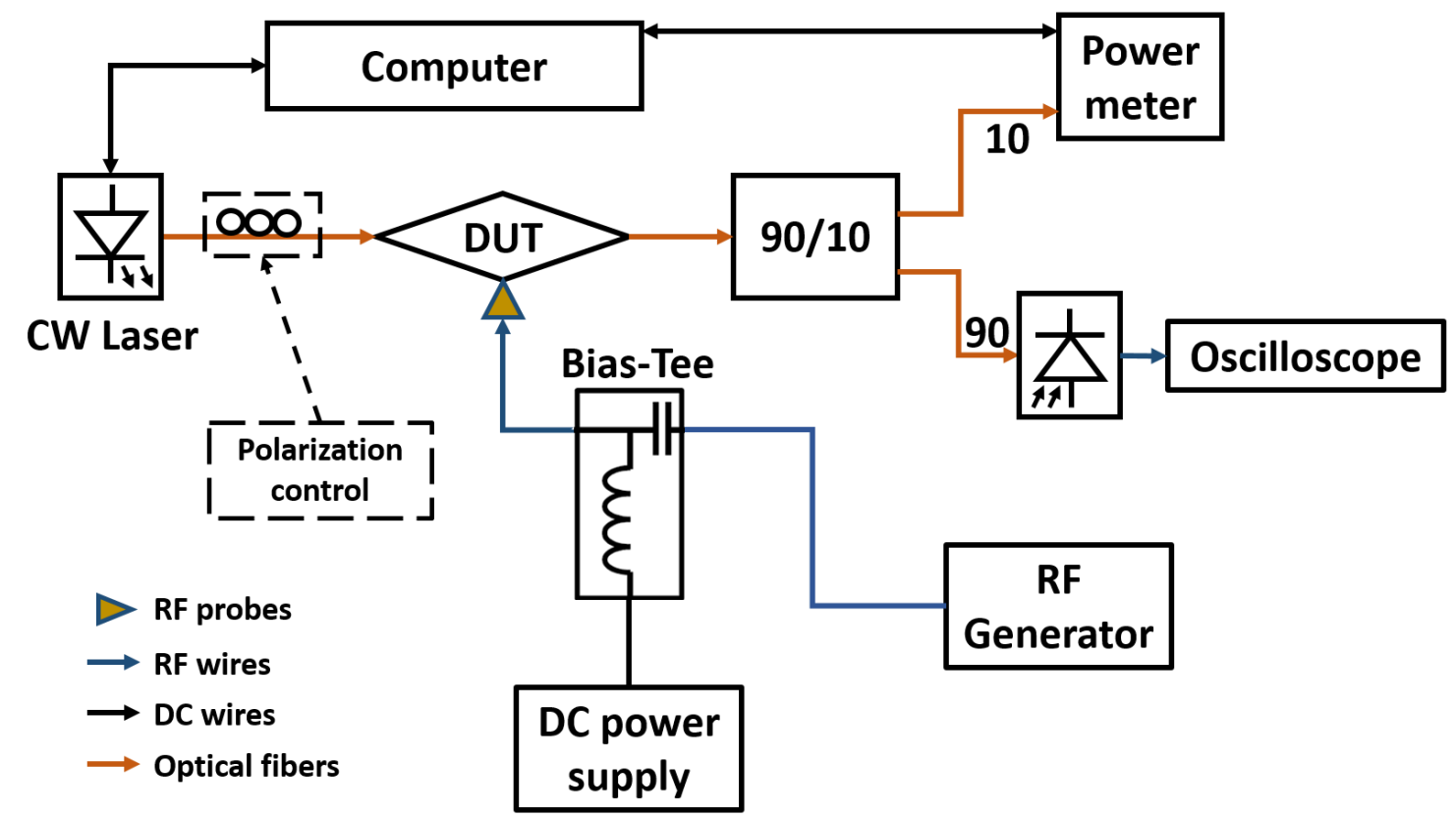

Figure 25. Set-up schematic for the switching time measurement of the tunable devices.

However, there will be a trade-off between switching time and power consumption depending on the MMI structure. A very fast switching time of $1.19 \mu$ s with a power consumption of $54.4 \mathrm{~mW}($ Р $\cdot \tau \approx 65 \mathrm{~mW} \cdot \mu \mathrm{s})$ has been achieved by means of the MMI structure without silica trench and meander heaters. The power consumption can be reduced to $24.9 \mathrm{~mW}$ by introducing the silica trench in the MMI structure but at expenses of a higher switching time of $4.25 \mu \mathrm{s}\left(\mathrm{P}_{\Pi} \cdot \tau \approx 108\right.$ $\mathrm{mW} \cdot \mu \mathrm{s})$. Figure 27-a shows the oscilloscope screen with the switching time for the tunable MMI with trench and meander heater and Figure 27-b shows the switching time for the MMI with trench and straight heater. Overall, the obtained results outperform the performance of MZI and MMI switches based also on thermo-optic effect with the heaters placement on top of the cladding layer [23][25], [27], [29] and [30], which performance has been summarized in Table 2. Summary of photonic switches performance based on thermo-optic effect. 


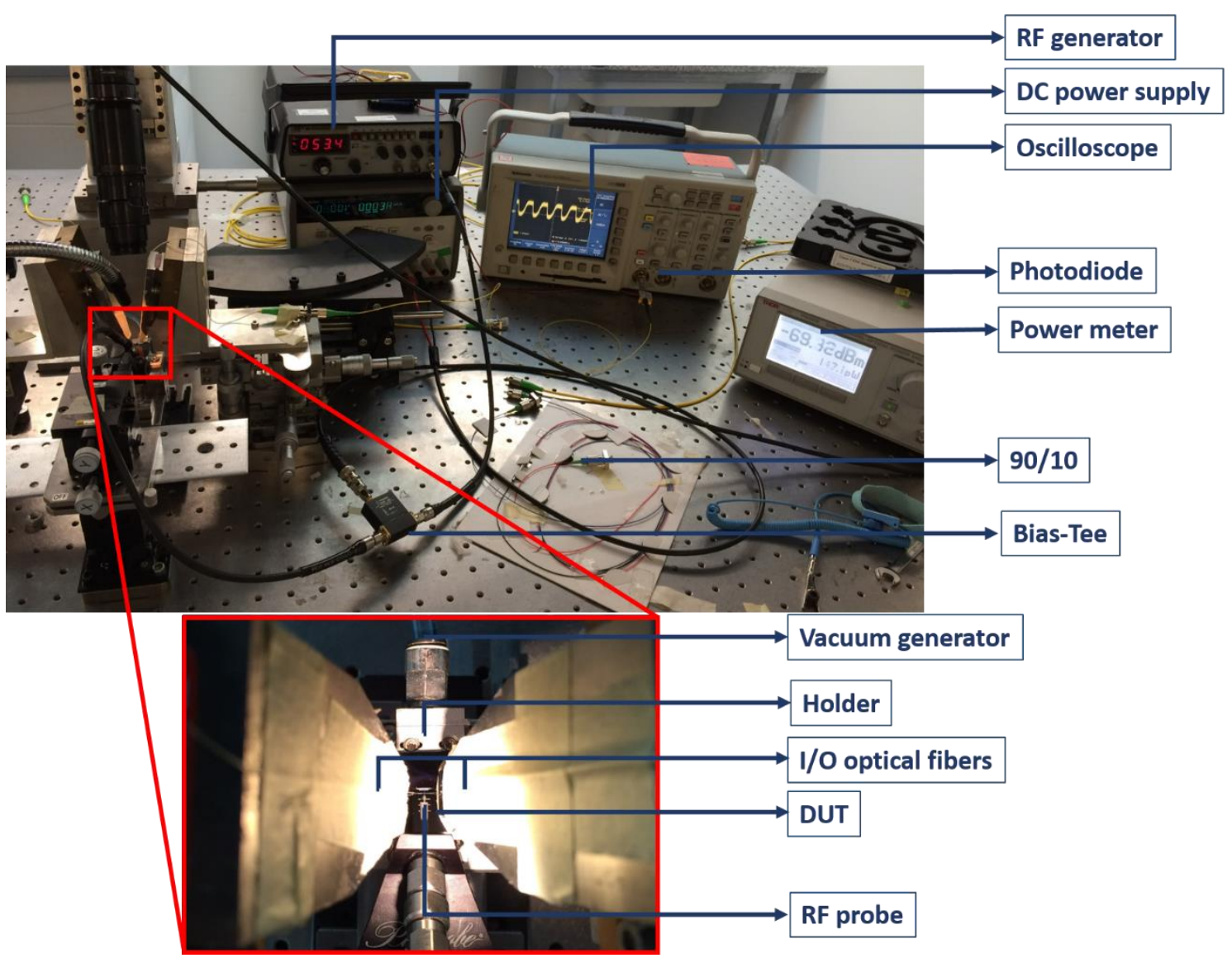

Figure 26. Pictures of the measurement set-up for the switching time of the tunable MMI.

\begin{tabular}{|c|c|c|c|c|}
\hline MMI structure & \multicolumn{2}{|c|}{ Without trench } & \multicolumn{2}{c|}{ With trench } \\
\hline Electrode structure & Straight & Meander & Straight & Meander \\
\hline Heating time & $0.53 \mu \mathrm{s}$ & $0.83 \mu \mathrm{s}$ & $0.45 \mu \mathrm{s}$ & $0.9 \mu \mathrm{s}$ \\
\hline Cooling time & $1.7 \mu \mathrm{s}$ & $1.19 \mu \mathrm{s}$ & $4.3 \mu \mathrm{s}$ & $4.25 \mu \mathrm{s}$ \\
\hline
\end{tabular}

Table 4. Measured switching time for the different structures

The best obtained figure of merit $\left(\mathrm{P}_{\Pi} \cdot \tau\right.$, where $\mathrm{P}_{\pi}$ is the power consumption and $\tau$ is the switching time) is also comparable to thermo-optic MZI switches based on doping the silicon to directly build the heaters into the waveguide $(\mathrm{P} \cdot \tau \approx 67 \mathrm{~mW} \cdot \mu \mathrm{s}$ in [28]). However, it should be pointed out that the fabrication complexity is notably increased as several implantation steps are required. In the proposed MMI switch, the fabrication is much simpler since the structure has a high robustness inherent to the MMI structure and the footprint is smaller than that of MZI-based switches. 


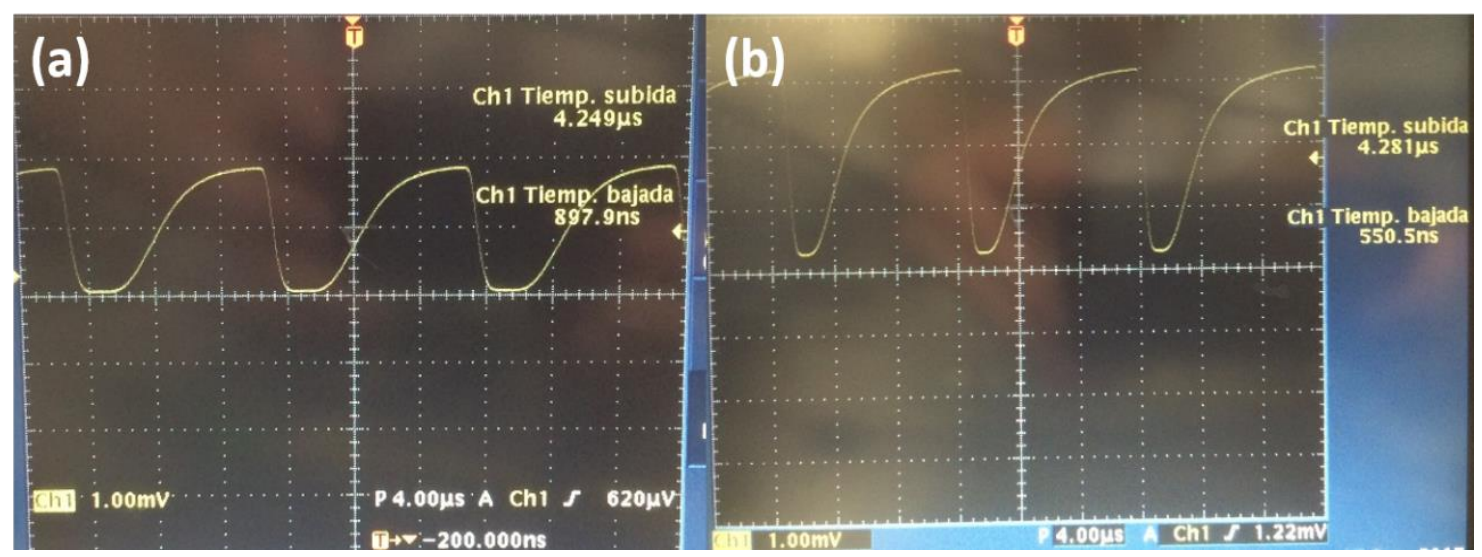

Figure 27. Pictures taken from oscilloscope for both MMI trench structure. (a) Shows the switching time corresponding to MMI trench with meander heater and (b) shows the switching time for MMI trench with straight heater.

\subsection{Application of micro-heater structures in $\mathrm{VO}_{2}$-silicon based switches}

\subsubsection{Vanadium dioxide introduction}

The integration of vanadium dioxide $\left(\mathrm{VO}_{2}\right)$ with silicon photonics allows the development of ultra-compact optical devices taking advantage of its reversible insulator-metal transition (Figure 28) through an external applied effect [41]. The insulator-to-metal transition is usually achieved by increasing the temperature above the critical value $\left(70^{\circ} \mathrm{C}\right)[42]$-[44]. The most useful way to combine $\mathrm{VO}_{2}$ with silicon photonics is by placing $\mathrm{VO}_{2}$ thin films over the silicon waveguide [45][48]. Therefore, it is possible to control the performance of photonic devices by means of the absorption change emerged from the $\mathrm{VO}_{2}$ insulator-metal transition. Placing the $\mathrm{VO}_{2}$ closer to the waveguide increases its effect over the optical field. To induce the phase transition thermally, a heater is placed near the vanadium dioxide in order to transfer the generated heat in an efficient way. Nevertheless, if the heater is positioned too close to the waveguide, optical losses would be induced due to the interaction between the optical field and the metal of the heater. 


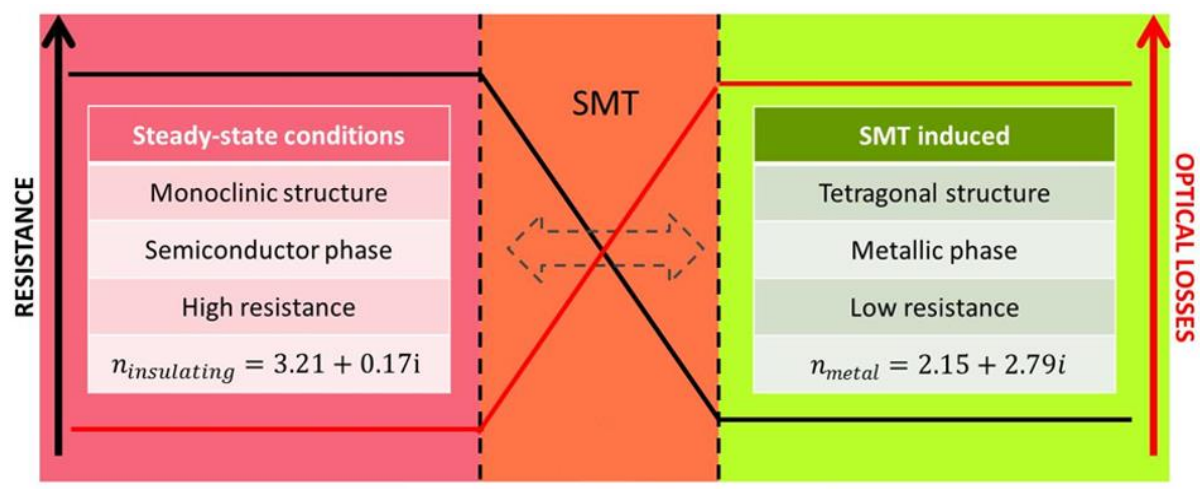

Figure 28. Summarize of vanadium dioxide characteristics

\subsubsection{Device description and method analysis}

In order to solve the losses issue, the most used solution to control the $\mathrm{VO}_{2}$ transition by means of a temperature increase, consists on depositing a silica gap between a heater and the hybrid $\mathrm{VO}_{2} / \mathrm{Si}$ waveguide, as it is shown in Figure 29a. The gap with a thickness around 700nm is used to avoid the introduction of optical losses by the metal, as it has been previously reported in Figure 29-b.
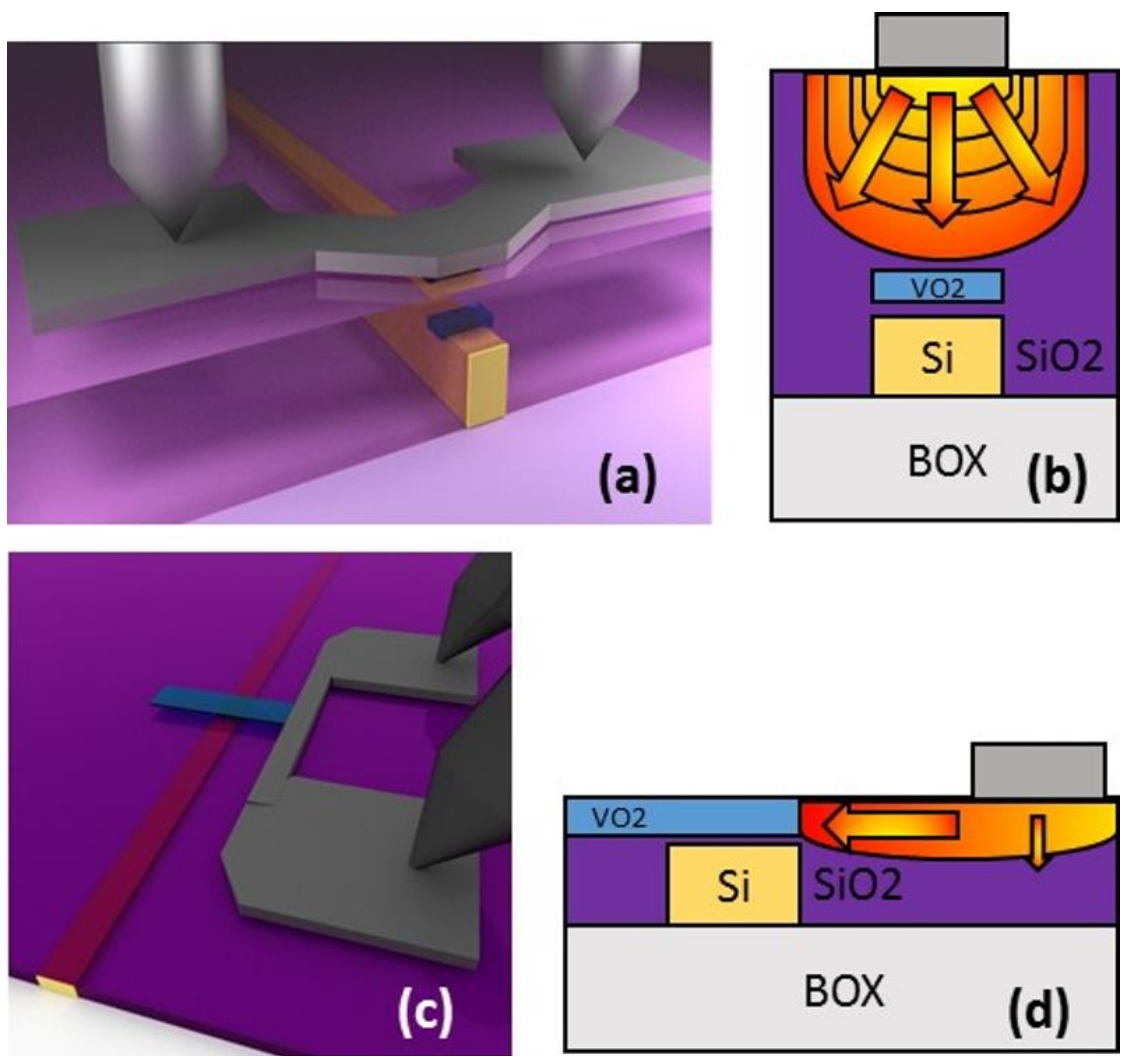

Figure 29. (a) Concept art and (b) cross section showing the heat distribution in a standard heating structure. (c) Lateral displaced micro-heater concept art and (d) cross section of the structure showing the generated heat guided along the VO2 layer due to its higher thermal conductivity [50]. 
The generated heat is propagated through the silica gap until it reaches the $\mathrm{VO}_{2}$, as it is shown in Figure 29-c. However, a lateral electrode configuration, as shown in Figure 29-c, is expected to improve the heat transfer process. In this case, the silica gap is not necessary because the metal can be placed close to the waveguide without adding optical losses. Due to the higher thermal conductivity of the vanadium dioxide in comparison with the silica, the generated heat is partially guided through the vanadium dioxide layer as it is schematized in Figure 29-d. Applying enough power to the heater, the generated heat switches the $\mathrm{VO}_{2}$ over the waveguide allowing the control of the optical field. A similar lateral configuration has also been recently proposed for silicon waveguides [49]. Both micro-heater configurations have been simulated considering a titanium heater with a length of $20 \mu \mathrm{m}$ and therefore, a lateral distance between the waveguide and the heater of 1 m (Figure 30-a) has been compared with the commonly used upper heater (Figure 30-b). The vanadium dioxide has been modelled with a thermal conductivity of $3.5 \mathrm{~W} /(\mathrm{m} \cdot \mathrm{K})$ and a heat capacity of $677 \mathrm{~J} /(\mathrm{kg} \cdot \mathrm{K})$ for the insulating state. Figure 30-c shows the simulation results. There is a temperature gradient from the heater towards the hybrid $\mathrm{VO}_{2} / \mathrm{Si}$ waveguide. To achieve the critical temperature over the $\mathrm{VO}_{2} / \mathrm{Si}$ waveguide for a lateral displaced electrode an electrical power above $8 \mathrm{~mW}$ is required. However, despite silica gap works as a thermal isolating barrier, the center of the heater could be placed just over the $\mathrm{VO}_{2} / \mathrm{Si}$ waveguide overwhelming the $\mathrm{SiO}_{2}$ isolation and achieving the critical temperature at $3 \mathrm{~mW}$. It must be noted that critical temperature in the Figure $30-\mathrm{c}$ is reached at $50^{\circ} \mathrm{C}$ due to environment temperature is supposed to be at $20^{\circ} \mathrm{C}$.
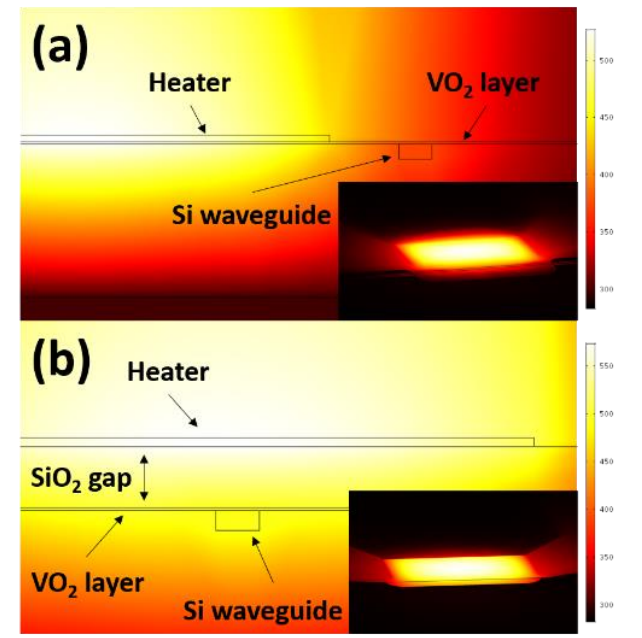

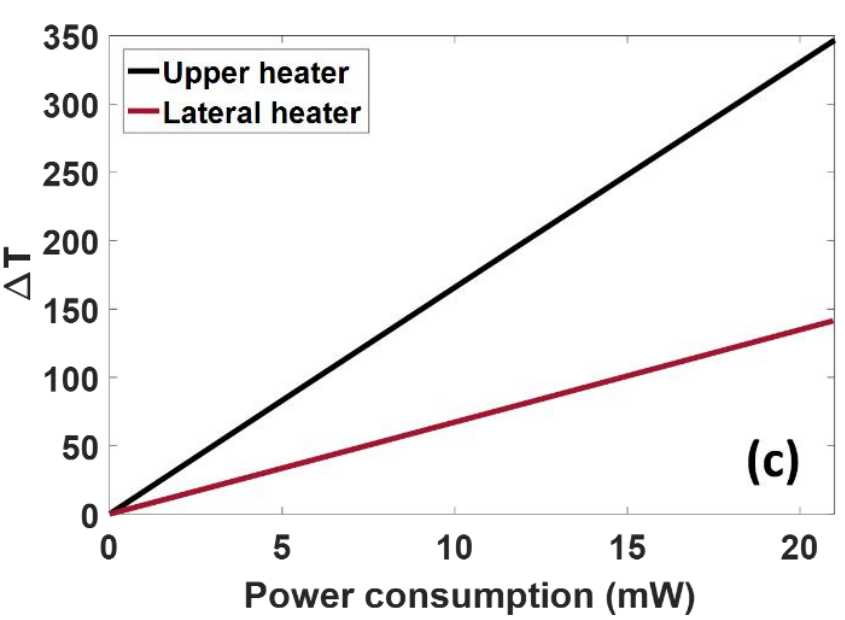

Figure 30. (a) Simulation of the heat dissipation for the lateral displaced heater. (b) Simulation of the heat dissipation for the upper heater. (c) Temperature variation as a function of the applied electrical power for both heater configurations. Furthermore, insets shown in (a) and (b) depicts a perspective image of the heater structure and the temperature distribution. 
Probably the trickiest step was the fabrication of $\mathrm{VO}_{2}$ layers, which was performed by our SITOGA partners of KUL Leuven. In such context, several samples were unable to be characterized. However, thanks to the effort of our partners, several structures with the lateral displaced micro-heater have been characterized for both light polarizations. Figure 31-a shows a SEM image of the tested structure. The electrode has been designed with a double metallization, (see section 2.5) to focus the generated heat in the proximities of the hybrid waveguide and has been placed over a continuous $\mathrm{VO}_{2}$ thin film as it is depicted in Figure 30-a and can be confirmed in the SEM image of the Figure 31-a. The variation of the optical power as a function of the applied electrical power for both input polarizations are shown in Figure 31-b and 31-c. Due to the topology of the hybrid section, the TM polarization has a higher interaction with the $\mathrm{VO}_{2}$ than the TE polarization, as it also can be deduced from Figure 31-b and 31-c. Therefore, the optical losses when the metallic state is reached are higher for TM than for TE polarization. Nevertheless, in both, the optical power is decreased when the applied power is around $8 \mathrm{~mW}$ for TE and $20 \mathrm{~mW}$ for TM. Furthermore, it should be noted that the gap in the fabricated sample with lateral displacement is around $550 \mathrm{~nm}$.
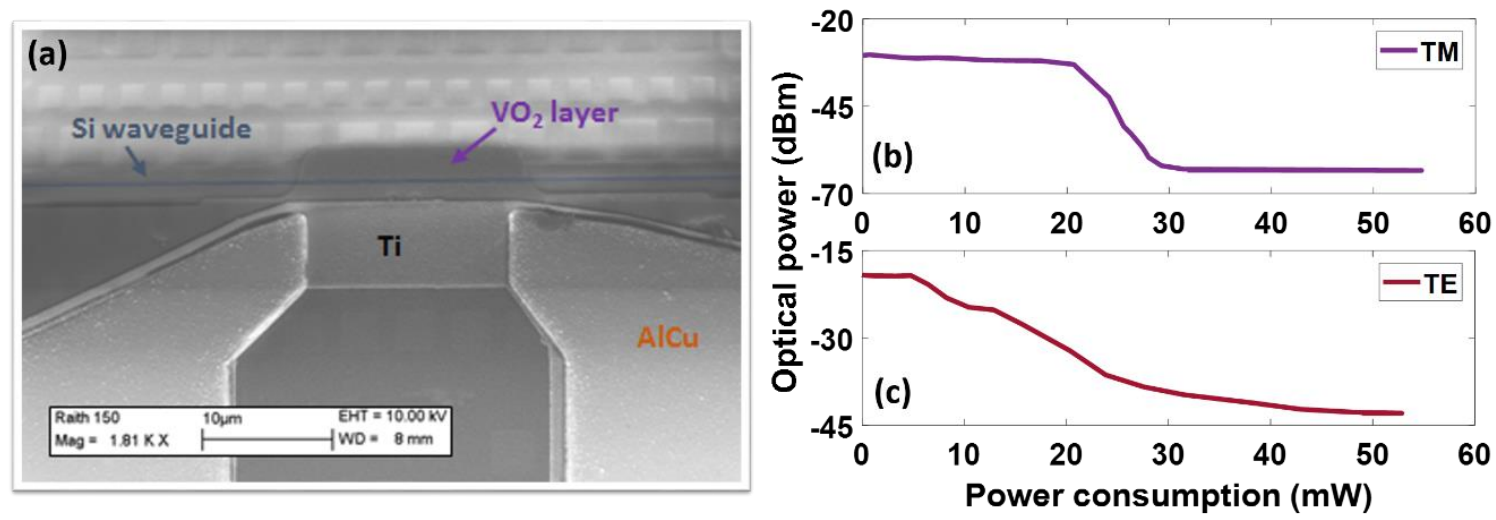

Figure 31. (a) SEM image of the fabricated hybrid waveguide structure with the lateral heater. (b) Variation of optical power as a function of the applied electric power TM polarizations and (c) for TE polarization.

Additionally, the switching time of the structure has been experimentally measured with the aim of achieving the fully characterization of the device. Figure 32 shows the oscilloscope screen with the obtained switching time. A values of $1.29 \mu \mathrm{s}$ for the fall time (heating) and $3.26 \mu \mathrm{s}$ for the rise time (cooling) has been obtained. The slow switching time in comparison with the previous results obtained with the tunable MMI could be attribute to the thermal conductivity of the $\mathrm{VO}_{2}$ and the proximity of the heater. 


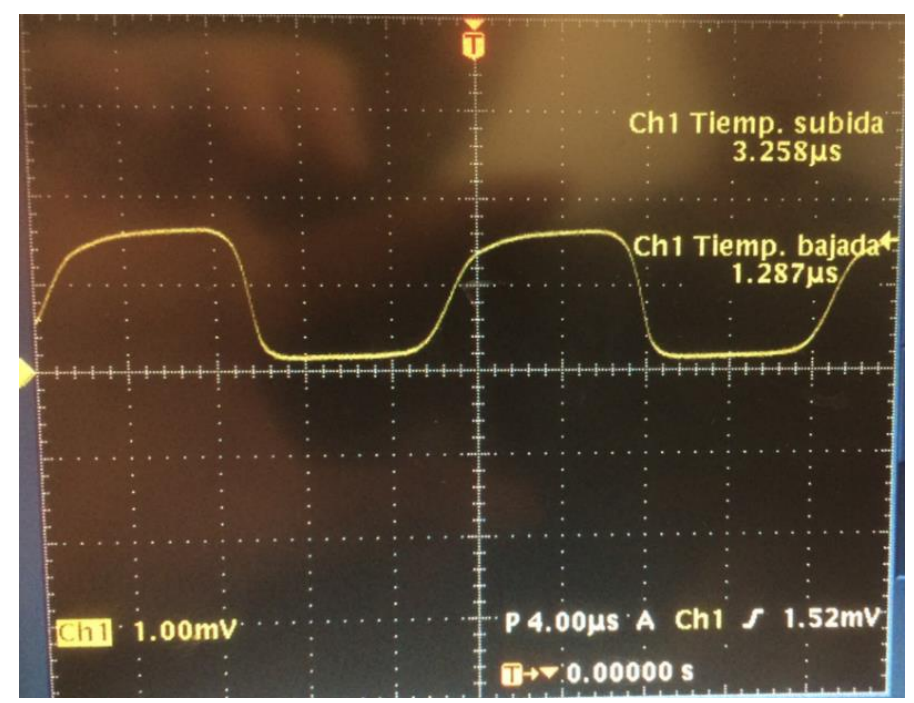

Figure 32. Obtained switching time for the hybrid $\mathrm{VO}_{2} / \mathrm{Si}$ waveguide switch.

\subsection{Conclusions}

In this chapter, silicon switches based on thermo-optically tunable MMI structures and hybrid $\mathrm{VO}_{2} / \mathrm{Si}$ waveguides have been demonstrated.

Thermal isolation in MMIs between adjacent self-images, optimized heater shapes and the use of different metals for the heater and pads have been shown to be key factor to minimize the power consumption. A trade-off between switching time and power consumption has also been obtained. Overall, such results place $2 \times 2$ optical MMI-based switches among of the state-of-the art of silicon photonic switches based on the thermo-optic effect with a power consumption of $24.9 \mathrm{~mW}$ and fast switching times of $1.19 \mu \mathrm{s}$.

Additionally, the combination of $\mathrm{VO}_{2}$ with silicon photonics has been demonstrated as a promising approach for the implementation of new active devices with ultra-compact footprint. Several applications require thermallyinduced phase transitions. In order to minimize the power consumption, a double metallization step has been performed. A lateral displaced micro-heater has been demonstrated to switch the $\mathrm{VO}_{2}$ between its different phases for both polarizations. Furthermore, an upper micro-heater configuration can be even more efficient according to the previously obtained simulation results.

Therefore, new routes have been proposed to address the implementation of switching devices in silicon photonic technology. 


\section{Chapter 3}

\section{Novel RF electrodes for application in optoelectronic traveling wave modulators.}

\subsection{Introduction and State-of-the-Art}

Since 1950 coplanar waveguides (CPWs) have awaken the interest of the electrical and scientific community due to their numerous advantages as a high frequency transmission line, its ease fabrication and cost-effective mass production as well as their easy integration with other circuital elements. CPWs can be very useful for a wide variety of applications due to their planar geometry (both ground and signal are in the same plane) which reduces their fabrication complexity and makes them compatible with a large variety of structures and applications. Furthermore, CPWs exhibit very low dispersion, and thus broadband performance, owing to their fundamental quasi-TEM propagation mode [51], [52]. Slow-wave CPWs can be viewed as an alternative to regular CPWs which allow the slowdown of the propagation velocity as well as the electrical length reduction [53]-[55]. Therefore, slow-wave CPWs are of paramount importance in several fields such us microwaves, photonics or plasmonics. In the microwave field, slowwave CPWs are used to design new compact delay lines [56], phase shifters or microwave filters [57]-[61] with an important size reduction in comparison with 
regular CPWs. In plasmonics, slow-wave CPWs have been used for designing and modelling new spoof surface plasmon modes [62]-[64]. Moreover, the management of the microwave index as well as the impedance is an essential target in metamaterials [65], [66] with several applications like compact multilayer transmission lines, negative and zero order resonator or lens design among others [66], [67]. Finally, regarding to the photonic field, slow-wave electro-optic modulators have been reported to reduce the drive voltage and footprint [9]-[13], [68]. However, slow wave CPWs are required for matching microwave and optical indices to avoid a reduction of the electro-optic modulation bandwidth [13].

Appropriate tuning of the microwave index of the slow-wave CPWs can therefore be beneficial in many fields and applications. The majority of works focus on increasing the capacitance of the CPWs as the main method to enlarge the microwave index [12], [59]-[61], [68]-[72]. Here, we propose an improved approach to properly design a high microwave index in slow-wave CPWs by increasing both capacitance and inductance. In such a way, we are able to demonstrate a gradual increase of the microwave index, reaching the highest value reported so far to the best of our knowledge. Furthermore, the influence of the capacitive and inductive elements on the impedance, to ensure a slow-wave CPW compatible with the standard $50 \Omega$ characteristic impedance, is also considered. The proposed slowwave CPWs features broadband performance with a bandwidth extending beyond $40 \mathrm{GHz}$.

\subsection{Coplanar waveguide theory}

To address the design of slow-wave CPWs, it is necessary to lay down the basis of microwave theory. This includes basically the transmission line theory to understand the transmission behavior, and the conformal mapping technique to analyze the influence of the different parameters in a CPW.

\subsubsection{Transmission line theory and background}

A coplanar waveguide is a specific kind of transmission line, so it can be studied with the commonly used transmission line theory.

Unlike direct current circuits, in high frequency transmission lines the voltage and current values varies along the propagation distance in amplitude and phase. Such variations cause the materialization of capacitive and inductive behaviors. Thus, 
a coplanar waveguide can be modelled as an equivalent circuit with equivalent values of capacitance, inductance, resistance and conductance, as it is depicted in Figure 33.
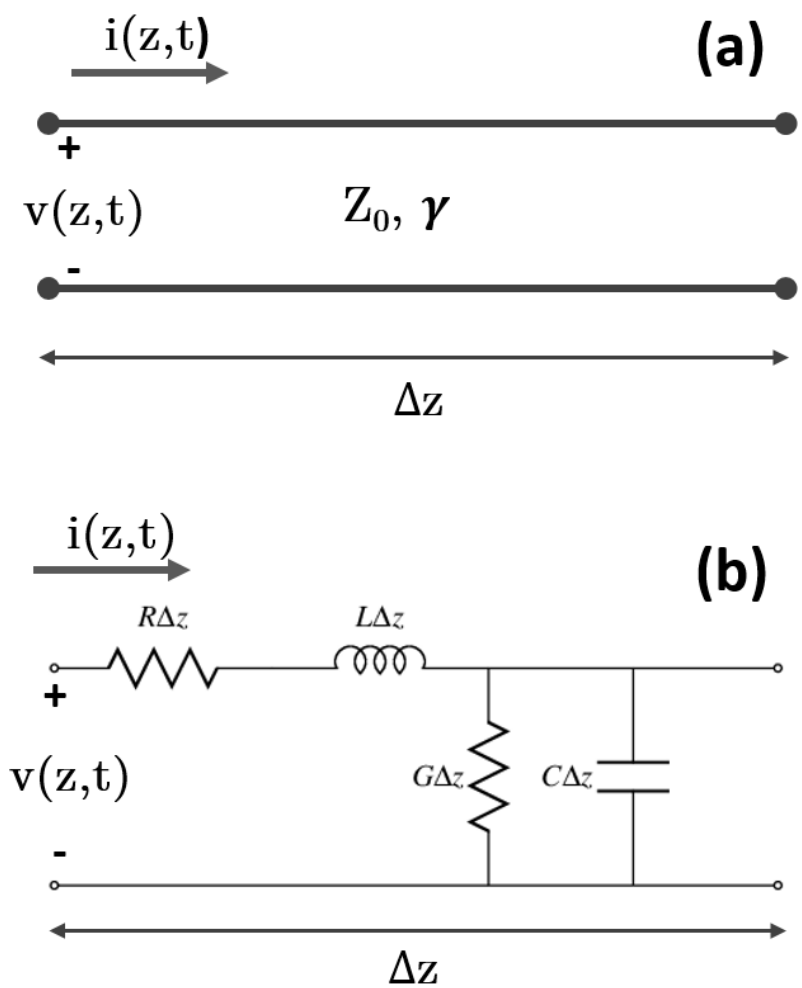

Figure 33. (a) Transmission line with its characteristic impedance and propagation constant. (b) Equivalent circuital model to the transmission line.

Applying the Kirchhoff's law in the equivalent circuit shown in Figure 33-b, the next equations can be obtained [52]:

$$
\begin{gathered}
v(z, t)-R \Delta z i(z, t)-L \Delta z \frac{\partial i(z, t)}{\partial t}-v(z+\Delta z, t)=0 \\
i(z, t)-G \Delta z i(z+\Delta z, t)-C \Delta z \frac{\partial v(z+\Delta z, t)}{\partial t}-i(z+\Delta z, t)=0
\end{gathered}
$$

By dividing the previous equation by the transmission line length $(\Delta \mathrm{z})$, and making the limits when $\Delta \mathrm{z} \rightarrow 0$, it is obtained:

$$
\begin{aligned}
& \frac{\partial v(z, t)}{\partial z}=-R i(z, t)-L \frac{\partial i(z, t)}{\partial z} \\
& \frac{\partial i(z, t)}{\partial z}=-G v(z, t)-C \frac{\partial v(z, t)}{\partial z}
\end{aligned}
$$


These equations are known as the telegraphist equations and describe the behavior of the transmission line in space and time domains. Assuming the transmission line has reached the steady state condition, the equations can be simplified to:

$$
\begin{aligned}
& \frac{d V(z)}{d z}=-(R+j \omega L) I(z) \\
& \frac{d I(z)}{d z}=-(G+j \omega C) V(z)
\end{aligned}
$$

From the previous Equation (3.5) and (3.6), the propagation equation can be obtained as:

$$
\begin{aligned}
& \frac{d^{2} V(z)}{d z^{2}}=\gamma^{2} V(z) \\
& \frac{d^{2} I(z)}{d z^{2}}=\gamma^{2} I(z)
\end{aligned}
$$

where the propagation constant is defined as:

$$
\gamma=\alpha+j \beta=\sqrt{(R+j \omega L)(G+j \omega C)}
$$

The voltages and currents in a transmission line can be therefore expressed as:

$$
\begin{gathered}
V(z)=V_{0}^{+} e^{-\gamma z}+V_{0}^{-} e^{\gamma z} \\
I(z)=I_{0}^{+} e^{-\gamma z}+I_{0}^{-} e^{\gamma z}
\end{gathered}
$$

where $e^{-\gamma z}$ represents the wave propagation in the positive direction $(+\mathrm{z})$ and $e^{+\gamma z}$ represents the wave propagation in the negative direction $(-z)$. Then, by applying Equation (3.5) in the Equation (3.10) it is obtained:

$$
I(z)=\frac{\gamma}{R+j \omega L}\left(V_{0}^{+} e^{-\gamma z}-V_{0}^{-} e^{\gamma z}\right)
$$


Finally, by dividing Equation (3.12) by the Equation (3.10) the impedance of the transmission line is expressed as:

$$
Z_{0}=\frac{R+j \omega L}{\gamma}=\sqrt{\frac{R+j \omega L}{G+j \omega C}}
$$

Once the impedance is found, from Equation (3.10) it is possible to come back to time domain in order to get the microwave index and propagation velocity relationship:

$$
v(z, t)=\left|V_{0}^{+}\right| e^{-\alpha z} \cos \left(\omega t-\beta z+\phi^{+}\right)+\left|V_{0}^{-}\right| e^{\alpha z} \cos \left(\omega t+\beta z+\phi^{-}\right)
$$

where $\phi^{ \pm}$is the phase of the complex voltage $V_{0}^{ \pm}$. Additionally, $\beta=2 \pi / \lambda$, $\omega=2 \pi f$ and the propagation velocity can be expressed as:

$$
v_{p}=\frac{\omega}{\beta}=\lambda f=\frac{\lambda_{0}}{n_{\mu}} f=\frac{c_{0}}{n_{\mu}}
$$

Therefore, as it can be seen there is an inversely proportional relationship between the propagation velocity and the microwave index, which means that for higher microwave indexes, the lower propagation velocity.

However, the analysis can be simplified assuming the lossless transmission line condition. Since transmission line losses are related with the resistance and the conductance, for a lossless transmission line it can be assumed that such parameters become negligible $(\mathrm{R}=\mathrm{G}=0)$. Thus, based on Equation (3.9) it can be obtained:

$$
\gamma=j \beta=\sqrt{(j \omega L)(j \omega C)}=j \omega \sqrt{L C}
$$

Furthermore, taking a look at the impedance in the Equation (3.13) and applying the lossless condition: 


$$
Z_{0}=\sqrt{\frac{L}{C}}
$$

Finally, if it is taken into account the new propagation constant from Equation (3.16) and applied on the Equation (3.15), it is obtained:

$$
v_{p}=\frac{c_{0}}{n_{\mu}}=\frac{\omega}{\beta}=\frac{1}{\sqrt{L C}}
$$

and the microwave index can be resolved as:

$$
n_{\mu}=c_{0} \sqrt{L C}
$$

Lossless transmission line analysis allows to understand the fundamental parameters management like the propagation constant, the impedance, the propagation velocity and the microwave index. Such characteristics are directly related with the inductive and capacitive elements of the equivalent circuit, and by modifying such elements, the characteristics parameters of the transmission line should be changed.

\subsubsection{Conformal mapping method}

The conformal mapping method allows to link the physical parameters of a coplanar waveguide with its fundamental parameters described above. Furthermore, the inductance and the capacitance can be obtained as a function of the length from the equivalent circuit parameters.

The analysis aims to be as general as possible, so it is going to considerate a coplanar waveguide over a dielectric substrate with dielectric constant $\boldsymbol{\varepsilon}_{\mathrm{r} 1}$ buried with another dielectric material with the dielectric constant $\boldsymbol{\varepsilon}_{r 2}$. Figure 34-a shows a concept art of a coplanar waveguide while Figure 34-b shows a transversal view with the main physical parameters of coplanar waveguides.

The conformal mapping method could also take into account the transmission losses related with the metal thickness, but for simplicity, we are going to consider the lossless case. Thus, such analysis allows to calculate the impedance $\left(\mathrm{Z}_{0}\right)$, the inductance (L) and the capacitance (C) of the coplanar waveguide. The 
capacitance is calculated as the sum of three capacitances, the one related with the substrate, the one related with the superstrate dielectric and the air capacitance [73].
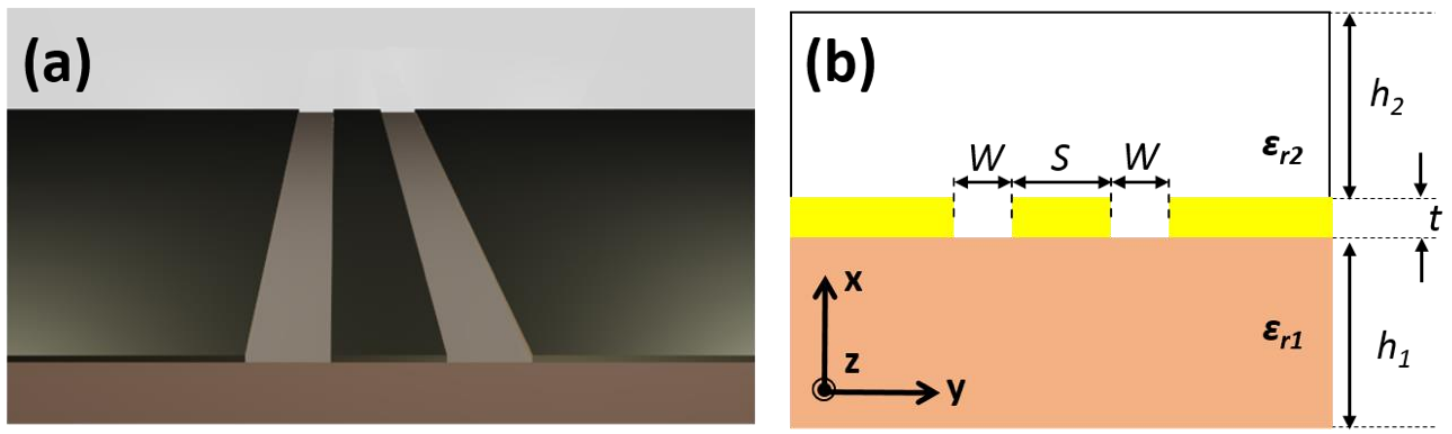

Figure 34. (a) Art concept of a coplanar waveguide and (b) transversal view of the CPW with the main physical parameters.

The capacitance related with the substrate $\left(\mathrm{C}_{1}\right)$ can be calculated as:

$$
C_{1}=2 \varepsilon_{0}\left(\varepsilon_{r 1}-1\right) \frac{K\left(k_{1}\right)}{K\left(k_{1}^{\prime}\right)}
$$

Where the module of the complete elliptical integral of the first kind $K(k)$ is defined as:

$$
\begin{gathered}
k_{1}=\frac{\sinh \left(\frac{\pi S}{4 h_{1}}\right)}{\sinh \left(\frac{\pi(S+2 W)}{4 h_{1}}\right)} \\
k_{1}^{\prime}=\sqrt{1-k_{1}^{2}}
\end{gathered}
$$

The capacitance related to the dielectric superstrate $\left(\mathrm{C}_{2}\right)$ is defined in a similar way than $\mathrm{C}_{1}$ in Equation (3.20), but with some differences in the module of the complete elliptical integral of the first kind:

$$
C_{2}=2 \varepsilon_{0}\left(\varepsilon_{r 2}-1\right) \frac{K\left(k_{2}\right)}{K\left(k_{2}^{\prime}\right)}
$$


where:

$$
\begin{gathered}
k_{2}=\frac{\sinh \left(\frac{\pi S}{4 h_{2}}\right)}{\sinh \left(\frac{\pi(S+2 W)}{4 h_{2}}\right)} \\
k_{2}^{\prime}=\sqrt{1-k_{2}^{2}}
\end{gathered}
$$

and finally, the air capacitance is defined as:

$$
C_{\text {air }}=4 \varepsilon_{0} \frac{K\left(k_{0}\right)}{K\left(k_{0}^{\prime}\right)}
$$

where:

$$
\begin{aligned}
& k_{0}=\frac{S}{S+2 W} \\
& k_{0}{ }^{\prime}=\sqrt{1-k_{0}^{2}}
\end{aligned}
$$

Thus, the total capacitance of the coplanar waveguide is:

$$
C_{C P W}=2 \varepsilon_{0}\left(\left(\varepsilon_{r 1}-1\right) \frac{K\left(k_{1}\right)}{K\left(k_{1}^{\prime}\right)}+\left(\varepsilon_{r 2}-1\right) \frac{K\left(k_{2}\right)}{K\left(k_{2}^{\prime}\right)}+2 \frac{K\left(k_{0}\right)}{K\left(k_{0}^{\prime}\right)}\right)
$$

The effective permittivity can be therefore defined as the total capacitance divided by the air capacitance, and can be written as:

$$
\varepsilon_{\text {eff }}=\frac{C_{C P W}}{C_{\text {air }}}=1+\left(\varepsilon_{r 1}-1\right) \frac{1}{2} \frac{K\left(k_{1}\right)}{K\left(k_{1}^{\prime}\right)} \frac{K\left(k_{0}^{\prime}\right)}{K\left(k_{0}\right)}+\left(\varepsilon_{r 2}-1\right) \frac{1}{2} \frac{K\left(k_{2}\right)}{K\left(k_{2}^{\prime}\right)} \frac{K\left(k_{0}^{\prime}\right)}{K\left(k_{0}\right)}
$$

Once the effective permittivity has been defined, the impedance can be found as: 


$$
Z_{0}=\frac{1}{C_{C P W} v_{p}}=\frac{30 \pi}{\sqrt{\varepsilon_{e f f}}} \frac{K\left(k_{0}{ }^{\prime}\right)}{K\left(k_{0}\right)}
$$

For the following steps it must be taken into account the relationship between the microwave index and the effective permittivity of an electromagnetic wave:

$$
n_{0}=\sqrt{\varepsilon_{e f f}}
$$

Regarding to the inductance, developing Equation (3.17) and replacing $\mathrm{Z}_{0}$ from Equation (3.31), it can be obtained:

$$
L=Z_{0}^{2} C_{C P W}=\left(\frac{1}{C_{C P W} v_{p}}\right)^{2} C_{C P W}=\frac{1}{C_{C P W}\left(\frac{c_{0}}{\sqrt{\varepsilon_{e f f}}}\right)^{2}}=\frac{\varepsilon_{e f f}}{C_{C P W} c_{0}^{2}}
$$

Therefore, by replacing the effective permittivity of Equation (3.33) for its definition in Equation (3.30) and then, the air capacitance described in Equation (3.26) it is finally obtained:

$$
L=\frac{\varepsilon_{\text {eff }}}{C_{C P W} c_{0}{ }^{2}}=\frac{1}{C_{a i r} c_{0}{ }^{2}}=\frac{1}{4 c_{0} \varepsilon_{0}} \frac{K\left(k_{0}{ }^{\prime}\right)}{K\left(k_{0}\right)}
$$

Thus, it is demonstrated that the inductance of the coplanar waveguide is only dependent on the central strip dimensions and the gap from the ground planes. The dielectrics surrounding the coplanar waveguide will not affect the inductance. In addition, the general study of conformal mapping presented can be particularized with the aim of make easier the mathematical analysis. Therefore, due to the CPW dimensions, the substrate and superstrate thickness can be considered infinite $\left(\mathrm{h}_{1}=\mathrm{h}_{2}=\infty\right)$, and, the superstrate permittivity can be replaced by the air permittivity $\left(\varepsilon_{\text {air }}=1\right)$.

In this way, the module of the complete elliptical integral of the first kind can be simplified to: 


$$
\begin{aligned}
& k_{1}=k_{0}=\frac{S}{S+2 W} \\
& k_{1}^{\prime}=k_{0}^{\prime}=\sqrt{1-k_{0}^{2}}
\end{aligned}
$$

Therefore, the total capacitance is only dependent on the air and the substrate and is expressed by the following equation:

$$
C_{C P W}=C_{1}+C_{a i r}=2 \varepsilon_{0}\left(\varepsilon_{r 1}-1\right) \frac{K\left(k_{1}\right)}{K\left(k_{1}^{\prime}\right)}+4 \varepsilon_{0} \frac{K\left(k_{0}\right)}{K\left(k_{0}^{\prime}\right)}=2 \varepsilon_{0}\left(\varepsilon_{r 1}+1\right) \frac{K\left(k_{0}\right)}{K\left(k_{0}^{\prime}\right)}
$$

The inductance, as it has been previously shown in Equation (3.34), do not depend on the dielectrics surrounding the CPW, so it remains unchanged for this case.

Thus, it can be clearly seen that conformal mapping is very useful due to the link provided between the physical parameters of the CPW and the circuital elements as well as other characteristic properties like CPW impedance and the effective permittivity, which is directly related with the microwave index, as well as the inductance and the capacitance of the CPW.

\subsection{Measurement methods}

There are different methods that can be used to analyze the results from the simulations or the measurements. This section is going to introduce such methods. The first method is based on the microwave index extraction from the scattering parameters. Since scattering parameters are required to be very precise for obtaining good results, this method is appropriate for post-simulation analysis. The second method is based on the microwave index extraction through a multiline method, which is more appropriate for experimental measurements.

\subsubsection{Microwave index extraction through ABCD matrix}

The transmission matrix ABCD allows to obtain the relationship between the scattering parameters and characteristic properties of a transmission line as inductance, capacitance, resistance, conductance, propagation constant, impedance or the microwave index. The next equation shows the relationship between the ABCD matrix and the scattering parameters matrix [52]: 


$$
\left[\begin{array}{ll}
A & B \\
C & D
\end{array}\right]=\left[\begin{array}{cc}
\frac{\left(1+S_{11}\right)\left(1-S_{22}\right)+S_{12} S_{21}}{2 S_{21}} & Z_{r} \frac{\left(1+S_{11}\right)\left(1+S_{22}\right)-S_{12} S_{21}}{2 S_{21}} \\
\frac{1}{Z_{r}} \frac{\left(1-S_{11}\right)\left(1-S_{22}\right)-S_{12} S_{21}}{2 S_{21}} & \frac{\left(1-S_{11}\right)\left(1+S_{22}\right)+S_{12} S_{21}}{2 S_{21}}
\end{array}\right]
$$

where $Z_{r}$ is the impedance source (usually $50 \Omega$ ). From the ABCD parameters the propagation constant can be determined as:

$$
\gamma=\frac{\ln \left(\frac{A+D}{2}+\sqrt{B C}\right)}{l}
$$

where $l$ is the length of the transmission line. Moreover, the characteristic impedance of the transmission line can be calculated as:

$$
Z_{0}=\sqrt{\frac{B}{C}}
$$

and therefore, the equivalent circuit elements are related with the inductance and the propagation constant in the following way:

$$
\begin{aligned}
& L_{0}=\frac{\operatorname{Im}\left(\gamma Z_{0}\right)}{\omega} \\
& C_{0}=\frac{\operatorname{Im}\left(\frac{\gamma}{Z_{0}}\right)}{\omega} \\
& R_{0}=\operatorname{Re}\left(\gamma Z_{0}\right) \\
& G_{0}=\operatorname{Re}\left(\frac{\gamma}{Z_{0}}\right)
\end{aligned}
$$

Thus, from the scattering parameters and taking into account the Equation (3.19) it is possible to obtain the microwave index of the transmission line. 
Additionally, microwave index can be also obtained from the phase of the scattering parameter $\mathrm{S}_{21}$ following the next relationship [74]:

$$
n_{\mu}=\frac{\varphi c_{0}}{\omega l}
$$

where $\omega$ is the angular frequency, $l$ is the length of the transmission line, $\varphi$ is the phase of $S_{21}$ parameter and $c_{0}$ is the light speed in vacuum.

\subsubsection{Microwave index extraction through multiline method}

The multi-line method [75], [76] allows to obtain the complex propagation constant $(\gamma)$ and the effective permittivity of the transmission line through the following equation $[9]$ :

$$
\varepsilon_{e f f}=-\left(\frac{\gamma c_{0}}{2 \pi f}\right)^{2}
$$

where $c_{0}$ is the speed of light in vacuum and $f$ is the RF frequency. One of the main advantages of this method is that it does not requires the calibration of the vector network analyzer (VNA) to measure the scattering parameters $\left(\mathrm{S}_{11}, \mathrm{~S}_{12}, \mathrm{~S}_{21}\right.$ and $\mathrm{S}_{22}$ ). To apply the method, the scattering parameters are first converted into transmission parameters:

$$
T=\frac{1}{S_{21}}\left(\begin{array}{cc}
-\Delta & S_{11} \\
-S_{22} & 1
\end{array}\right)
$$

where:

$$
\Delta=S_{11} S_{22}-S_{12} S_{21}
$$

The use of transmission matrices instead of scattering parameters has the advantage of exploiting the cascade multiplying property. Let the transmission matrices of the thru (reference line with shortest length) and of the longer delay lines to be denoted as $T_{t}$ and $T_{d}$ respectively. $T_{1}$ and $T_{2}$ represent the error 
matrices introduced in each port of the uncalibrated VNA. Thereby, the measured transmission matrices can be expressed as:

$$
\begin{gathered}
T_{t}=T_{1} T_{2} \\
T_{d}=T_{1} T_{l} T_{2}
\end{gathered}
$$

where Tl represents the delay line without the error:

$$
T_{l}=\left(\begin{array}{cc}
e^{-\gamma \Delta l} & 0 \\
0 & e^{\gamma \Delta l}
\end{array}\right)
$$

and $\Delta \mathrm{l}$ represents the length difference between thru and the respective delay line. The following matrix elements can be calculated from the measured matrices by:

$$
\left(\begin{array}{ll}
t_{11} & t_{12} \\
t_{21} & t_{22}
\end{array}\right)=T_{d} T_{t}^{-1}
$$

such that the propagation constant can be therefore calculated as:

$$
\gamma=\frac{1}{2 \Delta l} \ln \left(\frac{t_{11}+t_{22} \pm \sqrt{\left(t_{11}-t_{22}\right)^{2}+4 t_{12} t_{21}}}{t_{11}+t_{22} \mp \sqrt{\left(t_{11}-t_{22}\right)^{2}+4 t_{12} t_{21}}}\right)
$$

where the influence of the error matrices, $T_{1}$ and $T_{2}$, has been suppressed [76]. The sign assignment in Equation (3.53) should be such that $\left|e^{2} \gamma^{1}\right|<1$. Therefore, the real part of the effective permittivity is obtained from Equation (3.46).

Finally, Figure 35 shows the simulation results of the microwave index of a slowwave coplanar waveguide (SW-CPW) obtained with the three method previously reported and it can be seen that the difference between them is almost negligible. 


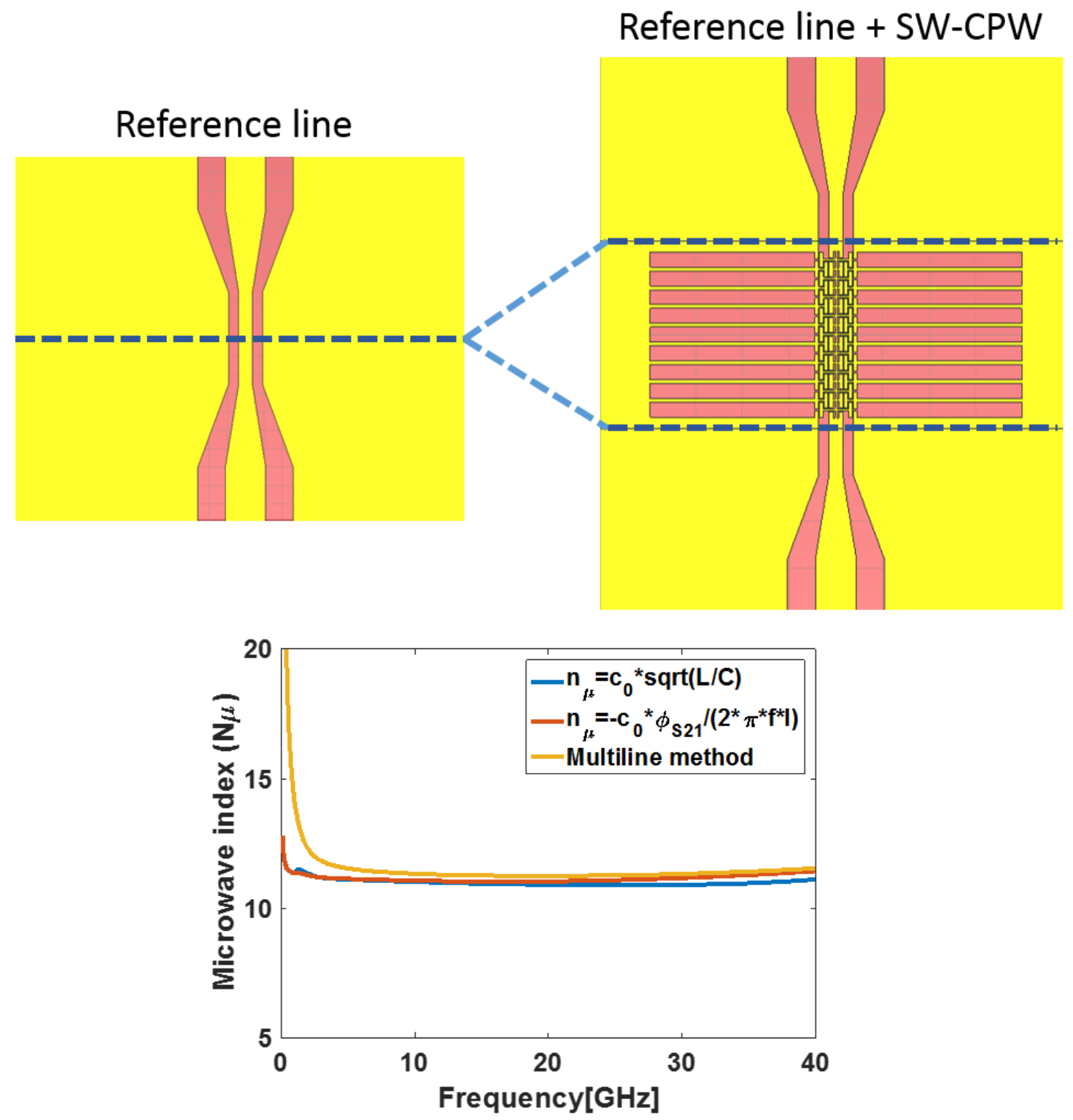

Figure 35. Reference line and SW-CPW inserted in the middle of the reference line in order to compare the different methods of microwave index extraction, as it can be seen in the graphic where the microwave index is plotted as a function of the frequency up to $40 \mathrm{GHz}$.

\subsection{Design of slow-wave coplanar waveguides}

In this point of the chapter, coplanar waveguide is going to be analyzed through the conformal mapping method with the aim of obtaining the impedance and the microwave index for a regular CPW of given dimensions. Figure 36 shows the influence of $\mathrm{W} / \mathrm{S}$ on the impedance and microwave index as well as on the inductance and capacitance for a silicon substrate $\left(\varepsilon_{\mathrm{r}}=11.9\right)$. 

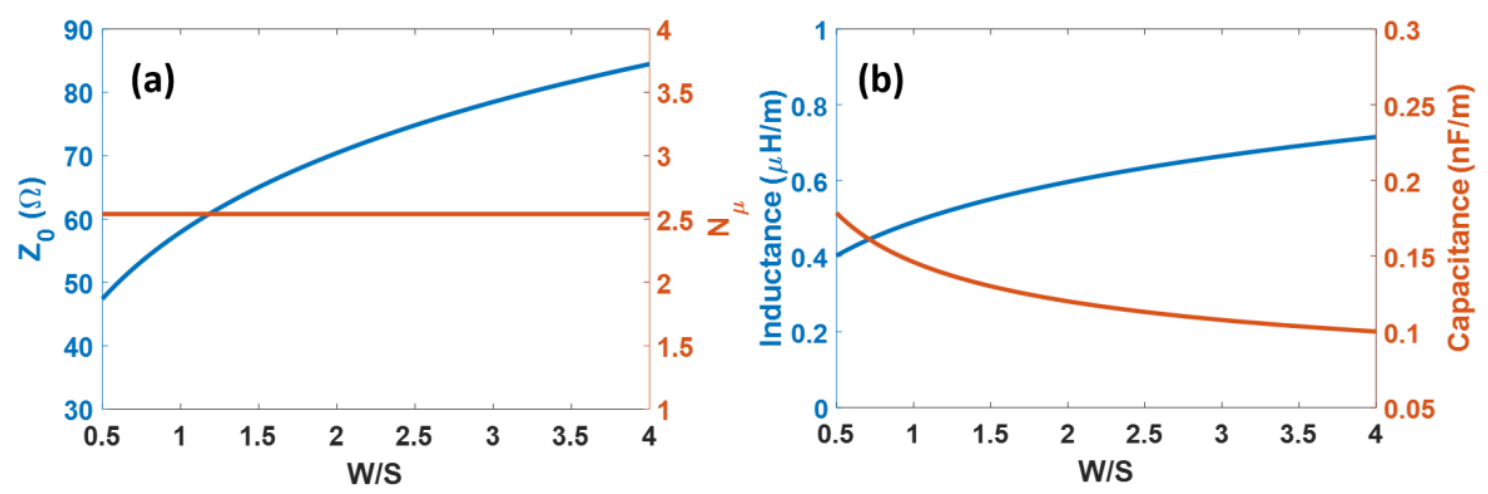

Figure 36. (a) Impedance and the microwave index, and (b) capacitance and the inductance as a function of W/S obtained by the conformal mapping method.

As can be seen in Figure 36-a, the impedance increases when W/S increases. However, the microwave index remains constant over the whole $\mathrm{W} / \mathrm{S}$ range because the reduction of the capacitance is balanced by the larger inductance (Figure 36-b). Thus, how can be the microwave index modified in coplanar waveguides?

In previous works [11], [60], [61], [68]-[71], the introduction of periodic capacitive elements (thin fins) to the CPW has been proposed to increase the capacitance without decreasing the inductance, which results in a microwave index increase and an impedance reduction. This is an effective method but does not take into account the inductance as a parameter that may also be exploited for the design.

In order to manipulate the inductance, the CPW strips can be reduced by using periodic thin slots. The proposed slots allow the inductance to be increased while only having a small effect on the capacitance. Therefore, increasing the inductance can be combined with approaches based on increasing the capacitance. Such combination will allow us to reach much higher microwave indices, while keeping the impedance close to $50 \Omega$.

The analysis has been carried out using the electromagnetic simulation software CST microwave studio. CST is a simulation tool that solves Maxwell equations for each point on a $2 \mathrm{D}$ or $3 \mathrm{D}$ mesh by using a finite elements method. Figure 36 shows the main interface of the CST microwave studio with a CPW, the electric field generated by a CPW and SW-CPW in Figure 37-b, 37-d and 37-f, and is corresponding scattering parameters in Figure 37-c, 37-e and 37-g respectively. The frequency sweep is up to $40 \mathrm{GHz}$, but taking advantage of the low dispersion in the $\mathrm{CPW}$, results at $20 \mathrm{GHz}$ are used with the aim of comparing the results obtained for the different simulated SW-CPW. 

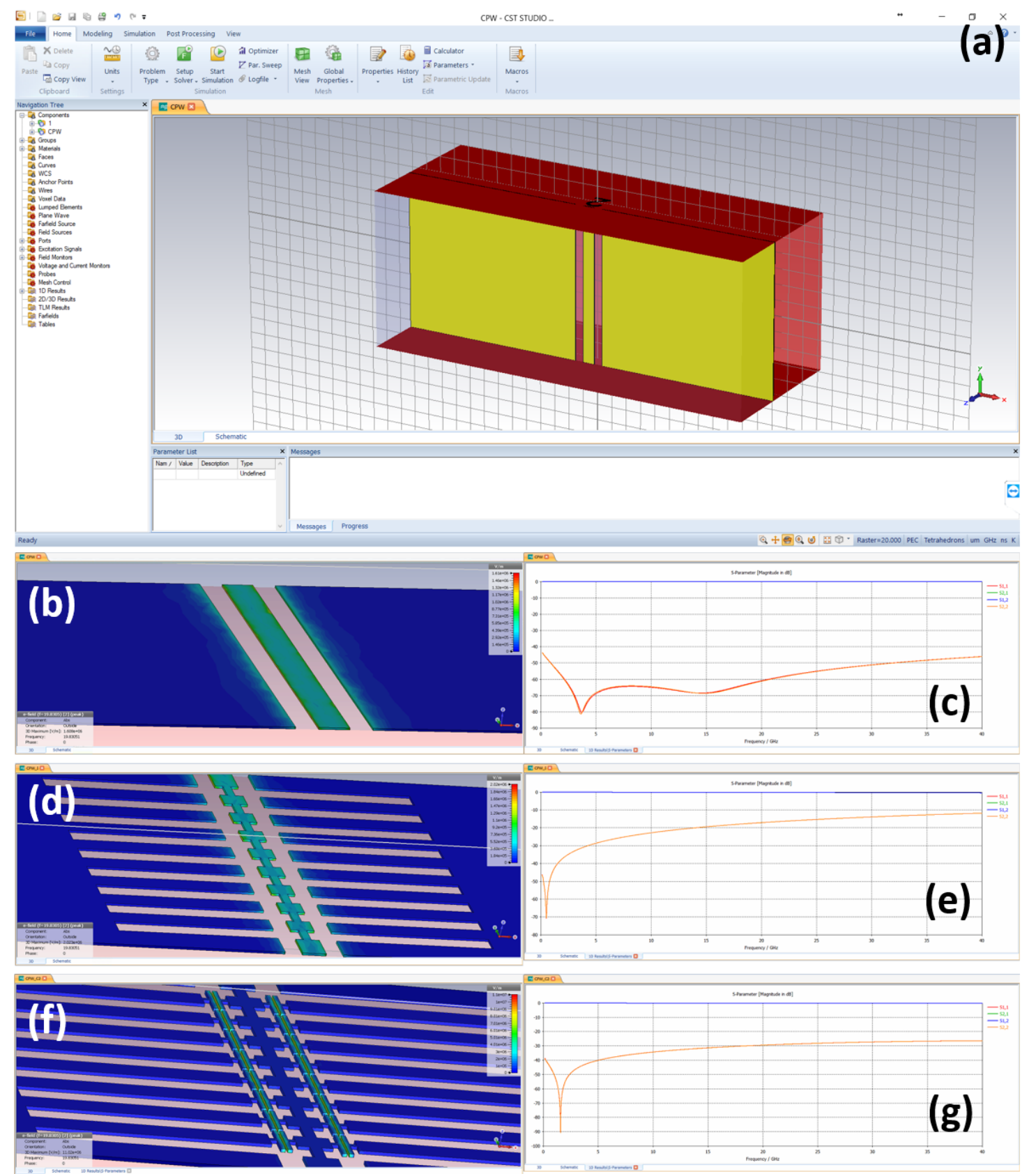

Figure 37. (a) Interface of CST with a CPW ready for a simulation, (b) electric field generated by a regular CPW and (c) their scattering parameters. (d) Electric field generated by a SW-CPW with inductive slots and (e) scattering parameters for the inductive a SW-CPW. Finally, (f) electric field generated by SW-CPW with inductive slots and capacitive elements and (g) scattering parameters of the capacitive SW-CPW.

To design the slow-wave CPW, it is crucial to analyze the thin slots in order to understand their influence on the inductance and the capacitance and therefore on the microwave index.

Figure 38-a shows a top view of the regular and slow-wave CPWs with the parameters to be designed. For the regular CPW, $\mathrm{S}=15 \mu \mathrm{m}$ and $\mathrm{W}=11 \mu \mathrm{m}$ have been chosen so that $\mathrm{W} / \mathrm{S}=0.73$. By properly checking Figure 38-a, an impedance 
value close to $50 \Omega$ is achieved. Furthermore, Figure 38-b depicts the proposed slots to increase the inductance, where $\mathrm{w}_{1}$ and $\mathrm{w}_{2}$ are the length and width of the slot. The period of the slots has been previously optimized to $20 \mu \mathrm{m}$. The variation of the capacitance and inductance as a function of $\mathrm{w}_{1}$ is shown in Figure 39-a for $\mathrm{w}_{2}=100 \mu \mathrm{m}$. It can be seen that there is an opposite behavior between them.
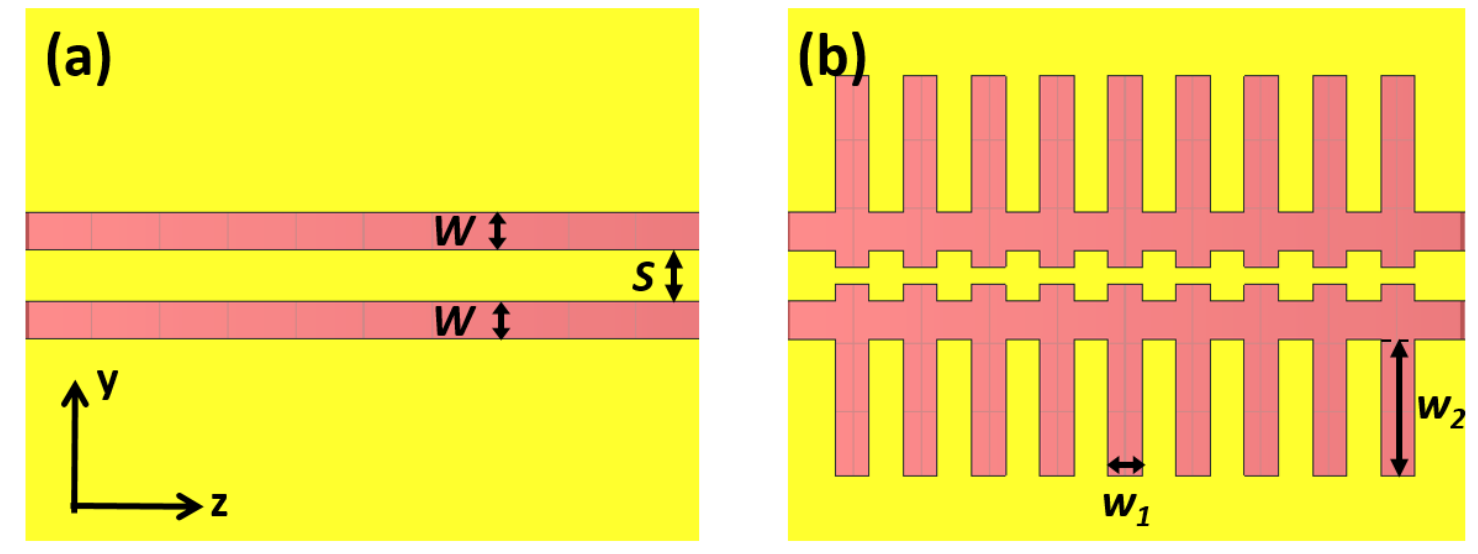

Figure 38. (a) Top view of a regular CPW and (b) of a slow-wave CPW.
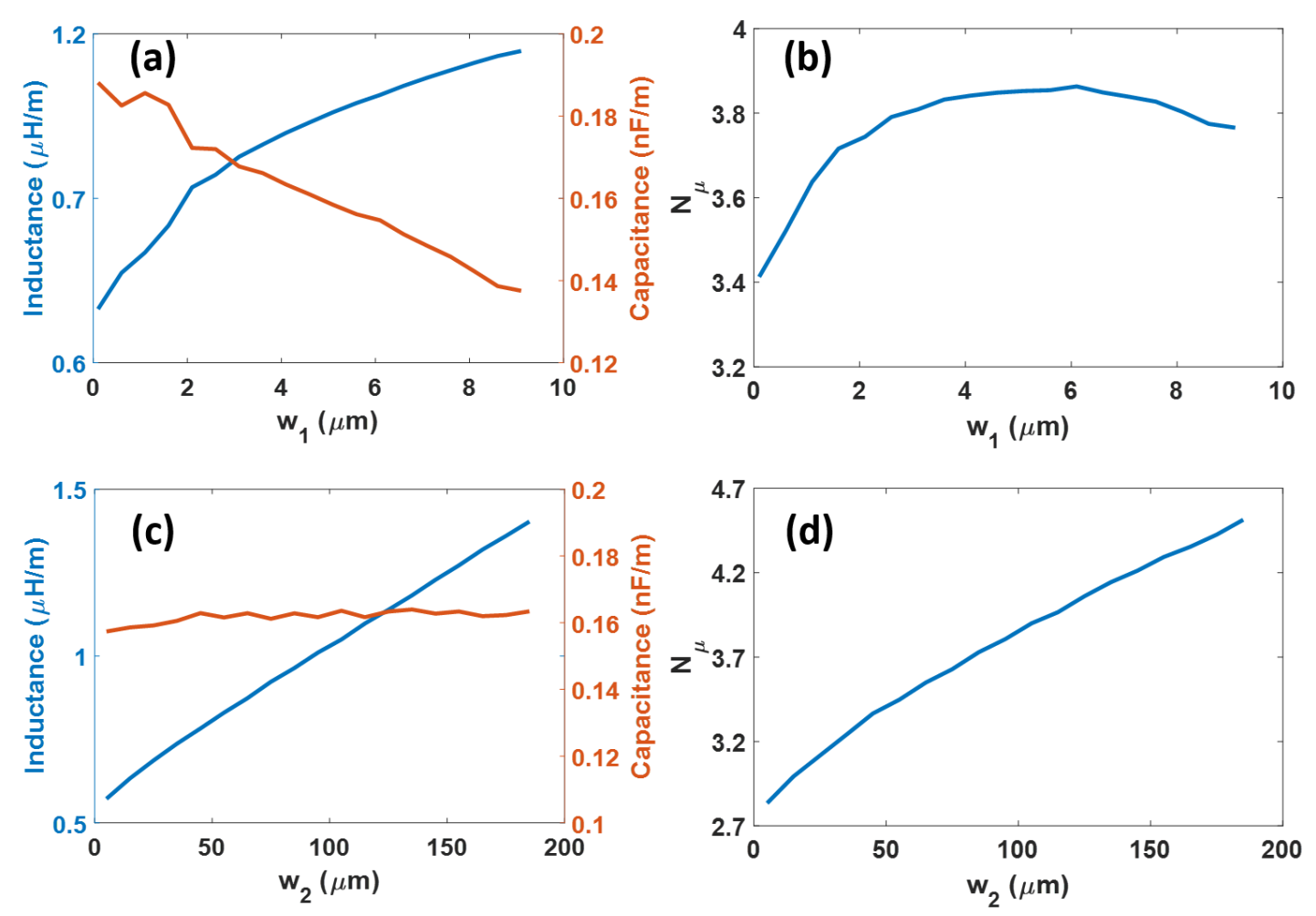

Figure 39. (a) Simulated inductance and capacitance and (b) microwave index as a function of w1 and $\mathrm{w} 2=100 \mu \mathrm{m}$ and (c) simulated inductance and capacitance and (d) microwave index as a function of $\mathrm{w}_{2}$ and $\mathrm{w}_{1}=5 \mu \mathrm{m}$ for the slow-wave CPW shown in Figure 37-b.

The variation of the capacitance and inductance as a function of $\mathrm{w}_{1}$ can be seen in the Figure 39-a for $\mathrm{w}_{2}=100 \mu \mathrm{m}$. It can be seen that there is an opposite behavior between them. The increase of the inductance is higher than the capacitance 
reduction, resulting in a microwave index increment (Figure 39-b). However, the increase of the inductance is not constant with $\mathrm{w}_{1}$ so that the increase of the microwave index is reduced for larger $\mathrm{w}_{1}$ values. On the other hand, the behavior of the capacitance and the inductance with $\mathrm{w}_{2}$ is shown in Figure $39-\mathrm{c}$, for $\mathrm{w}_{1}=5$ $\mu \mathrm{m}$. While the inductance increases with $\mathrm{w}_{2}$, the capacitance remains constant, which gives rise to higher microwave index with larger $\mathrm{w}_{2}$, as it can be seen in Figure 39-d.
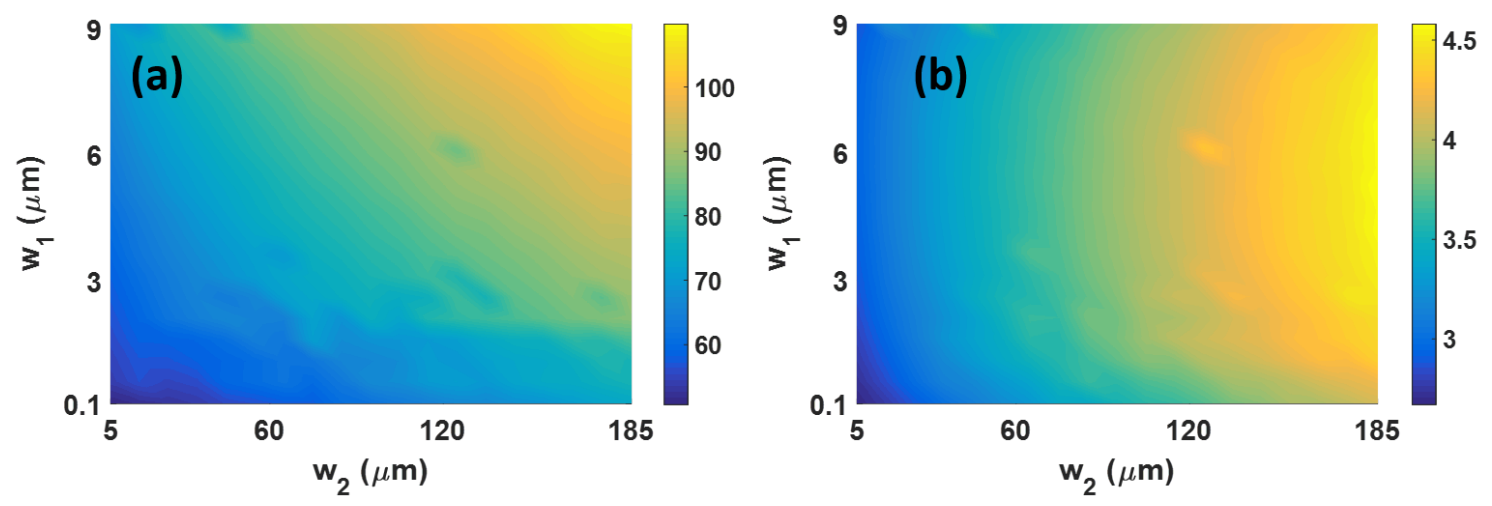

Figure 40. (a) Impedance and (b) microwave index as a function of $\mathrm{w}_{1}$ and $\mathrm{w}_{2}$.

Figure 40-a shows the impedance as a function of $\mathrm{w}_{1}$ and $\mathrm{w}_{2}$. The corresponding microwave index is depicted in Figure 40-b. It can be seen that larger microwave indices are achieved at expenses of increasing the impedance, which is in agreement with Equation (3.17) as the inductance increase dominates over the capacitance. Therefore, it is clear that the proposed slots act as enhanced inductive elements in the slow-wave CPW. However, to keep the impedance close to $50 \Omega$ without reducing the microwave index, the introduction of capacitive elements is also required. The capacitance can be increased with the introduction of parallel T-rails, as shown in Figure 41-a, which are periodically repeated along the propagation direction [12], [13], [68], [69]. A novel approach based on a crossed T-rail, as depicted in Figure 41-b, is here also proposed to further increase the capacitance. The induced electric field due to the T-rails is represented with blue lines in Figure 41-a, 41-b and 41-c.

Parallel T-rails with a length of $18 \mu \mathrm{m}$, width of $2 \mu \mathrm{m}$ and gap of $1 \mu \mathrm{m}$ have been added on the inductive slow-wave CPW with $\mathrm{w}_{1}=5 \mu \mathrm{m}$ and $\mathrm{w}_{2}=100 \mu \mathrm{m}$. The slow-wave CPW is shown in Figure 41-d and has been named as SW1. The same inductive slow-wave CPW but with the crossed T-rails has also been considered to evaluate the difference in capacitance and so the influence on the microwave index. In this case, the CPW is depicted in Figure 41-e and has been named as SW2. 

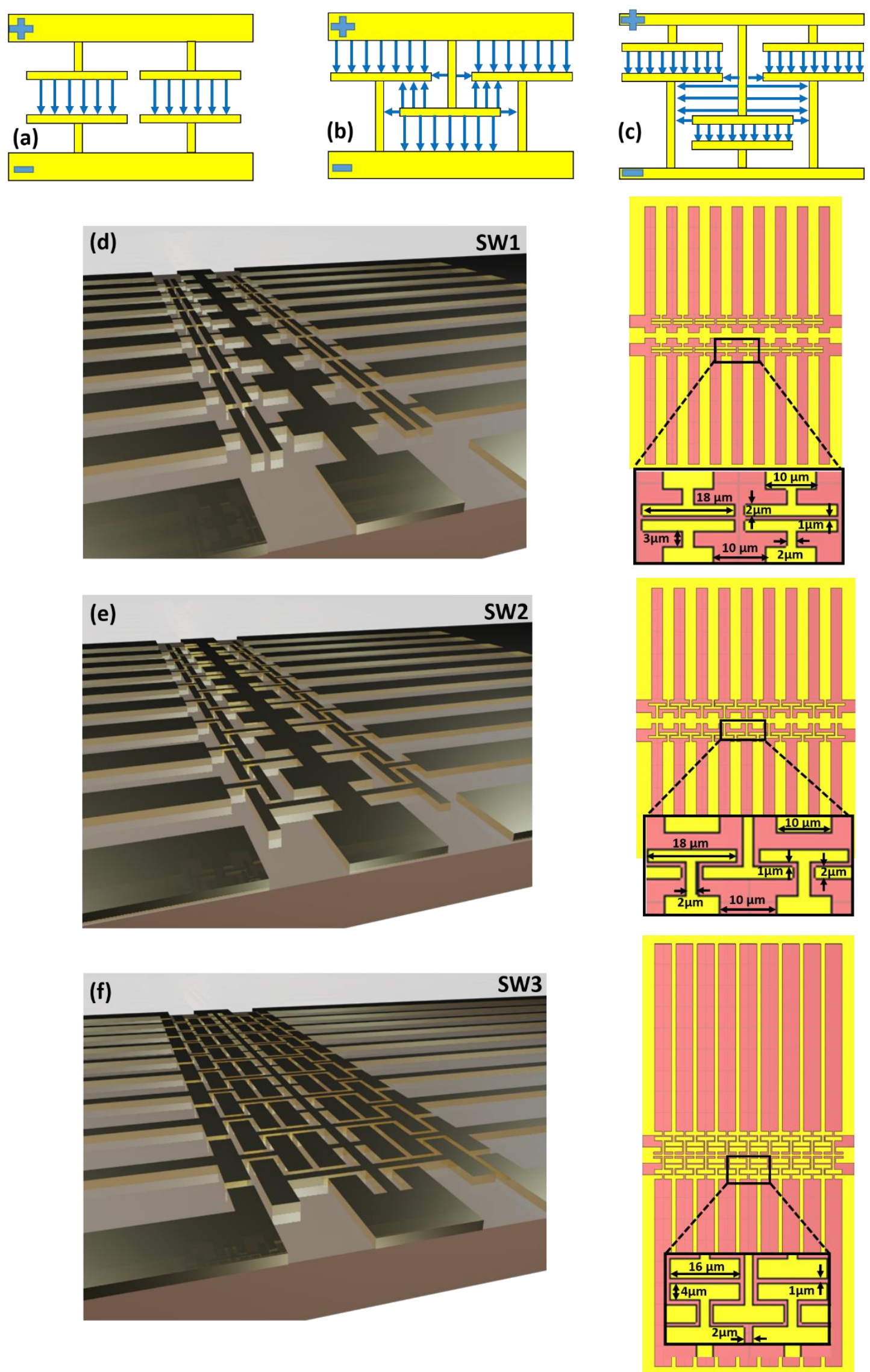

Figure 41. Schematic of (a) parallel and (b) novel crossed T-rails to increase the capacitance of the CPW. The blue lines represent the induced electric field due to the T-rails. Slow-wave CPW with (c) parallel Trails, (d) crossed T-rails and (e) a combination of both configurations for improved performance. 
Finally, a slow-wave CPW with a combination of both crossed and parallel Trails, named as SW3 and shown in Figure 41-f, has also been designed to further improve the capacitance. The length and width of the T-rails have been optimized to $16 \mu \mathrm{m}$ and $4 \mu \mathrm{m}$, respectively. Furthermore, the inductive performance has been enhanced by changing $\mathrm{w}_{1}$ to $17 \mu \mathrm{m}$ and $\mathrm{w}_{2}$ to $180 \mu \mathrm{m}$. As the capacitance of the slow-wave CPW is ruled by the capacitive T-rail elements, the reduction of the capacitance due to the larger $\mathrm{w}_{1}$ is negligible.
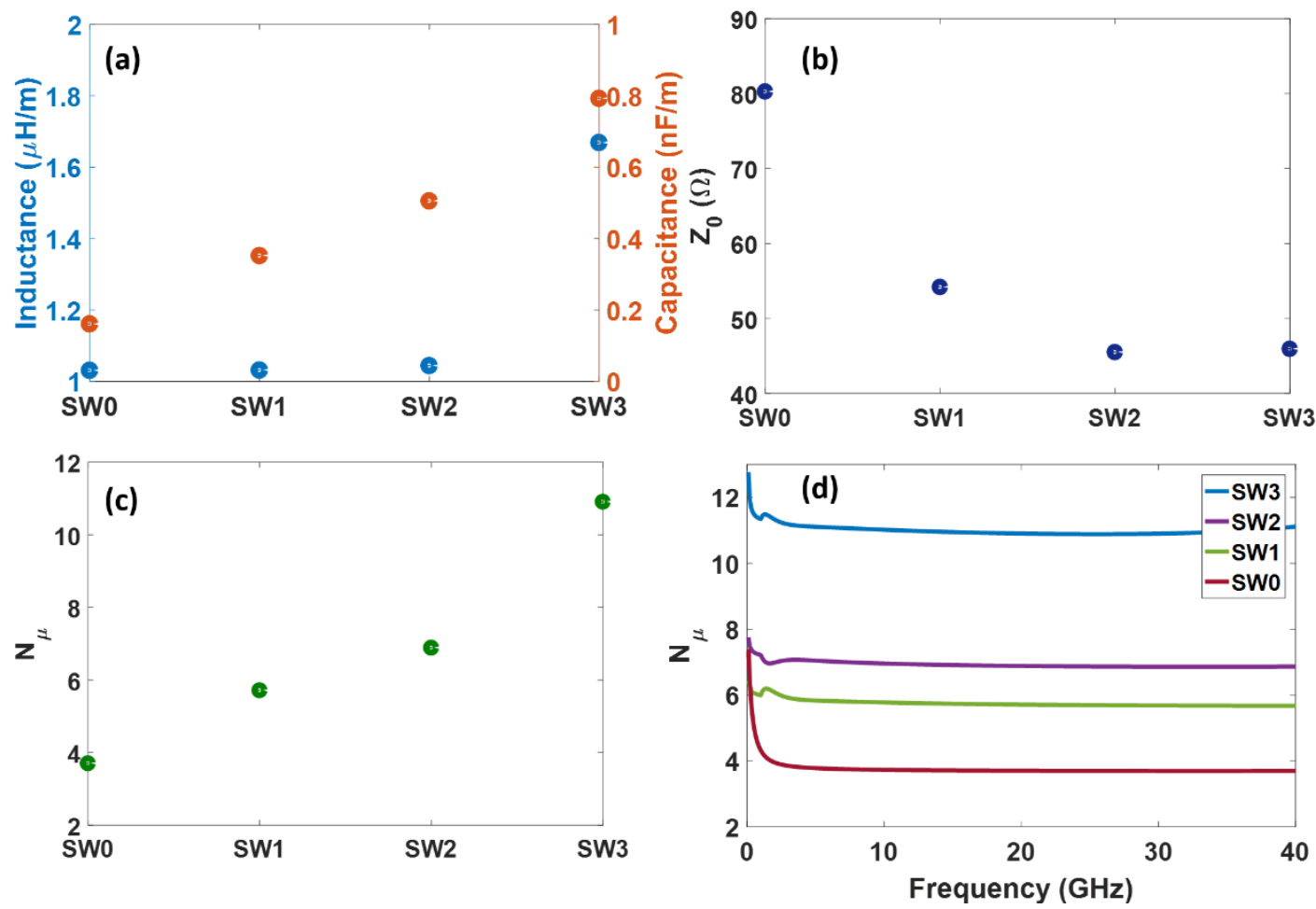

Figure 42. (a) Inductance and capacitance, (b) impedance and (c) microwave index for the different designs of the slow-wave CPWs obtained by simulations at $20 \mathrm{GHz}$. (d) Simulated microwave index as a function of the frequency.

Figure 42 shows the simulation results for the different designs of the slow-wave CPWs. SW0 refers to the inductive slow-wave CPW shown in Figure 37-b with $\mathrm{w}_{1}=5 \mu \mathrm{m}$ and $\mathrm{w}_{2}=100 \mu \mathrm{m}$. The capacitive and inductive behavior of the slowwave CPWs are depicted in Figure 42-a. The capacitance is gradually improved up to a factor of four (from 0.2 to $0.8 \mathrm{nF} / \mathrm{m}$ ) by the introduction of the capacitive T-rail elements in SW1 and their modifications in SW2 and SW3. The inductance is approximately constant for SW0, SW1 and SW2, but it increases from 1.05 to $1.67 \mathrm{\mu H} / \mathrm{m}$ for SW3 as a result of the changes in the ground slots and the modification of the signal strip. The impedance, Figure 42-b, and microwave index, Figure 42-c, will be determined by the capacitive and inductive behavior. The impedance is decreased to a value close to $50 \Omega$ for the improved designs of 
the slow-wave CPWs because of the larger capacitance with respect to the original design of SW0. The microwave index is also increased for SW1 and SW2 due to the larger capacitance but the improvement is much larger for SW3 as a result of the additional increase of the inductance. Thereby, the microwave index is significantly enhanced from 6.9 in SW0 up to 11 in SW3. The frequency response has also been simulated and is shown in Figure 42-d. A constant microwave index and therefore wideband operation is achieved due to the low dispersion of the quasi-TEM propagation mode in the slow-wave coplanar waveguide.

\subsection{Fabrication and characterization of slow- wave coplanar waveguides}

In order to demonstrate the simulated performance, some of the previously designed slow-wave CPWs have been fabricated and characterized. The devices were fabricated on a silicon substrate covered with a $300 \mathrm{~nm}$ thick $\mathrm{SiO}_{2}$ layer deposited using a plasma enhanced chemical vapor deposition (PECVD) process. The CPWs were formed with a lift-off process using TI35E photoresist in an image reversal process. The electrodes consist of $40 \mathrm{~nm} \mathrm{Ti}$, deposited through sputtering and $1000 \mathrm{~nm} \mathrm{Au}$, deposited through thermal evaporation. Figure 43 shows the schematic of the set-up for the experimental measurements as well as some real pictures of the ongoing measurements.

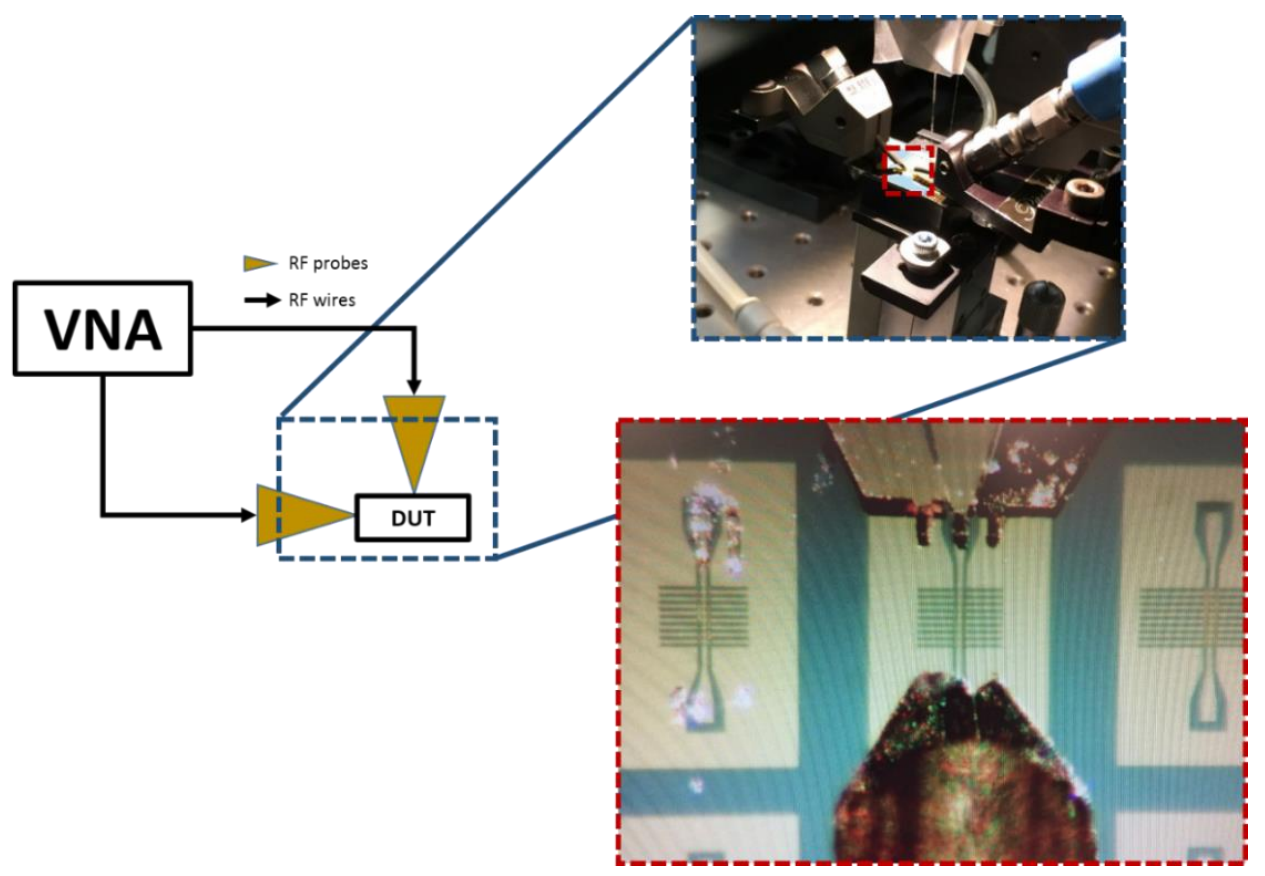

Figure 43. Schematic and images of the experimental set-up. Additionally, and image of the optical microscope where the probes are placed over a SL-CPW. 
Figure 44 shows the obtained measurements results and the comparison with simulations. A multiline method has been applied to extract the microwave index from the slow-wave part of the CPW. The method uses a reference CPW (inset of Figure 44-a) in addition to the slow-wave CPW and has been explained in detail in section 3.3.2.
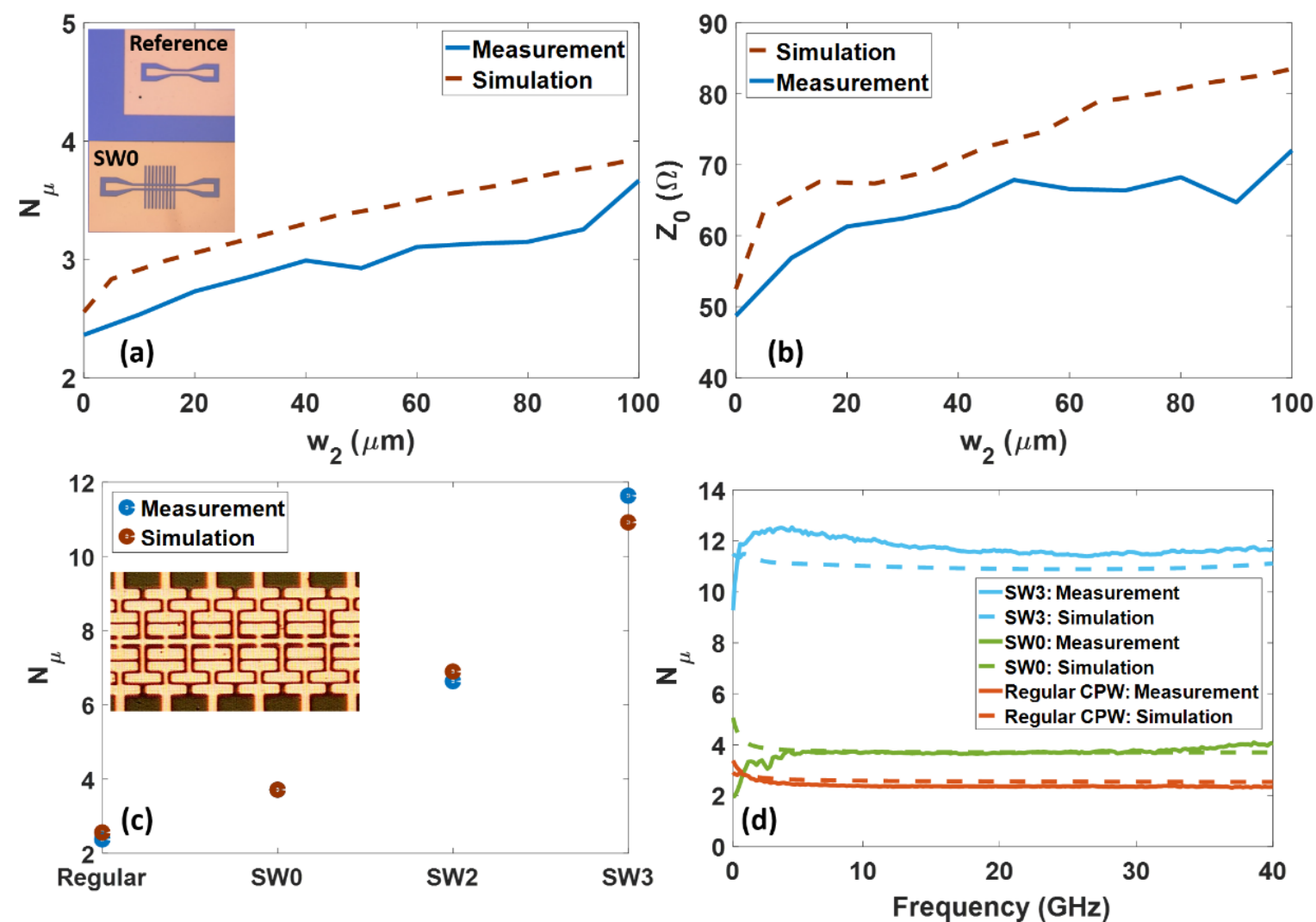

Figure 44. (a) Simulated and measured microwave index and (b) impedance for SW0 as a function of w2 and assuming $\mathrm{w}_{1}=5 \mu \mathrm{m}$. An inset with an optical view of the reference CPW and SW0 with $\mathrm{w}_{2}=100 \mu \mathrm{m}$ is included. Simulated and measured microwave index (c) at $20 \mathrm{GHz}$ for the regular CPW, SW0, SW2 and SW3 and (d) as a function of frequency for the regular CPW, SW0 and SW3. The inset in (c) shows an optical view of the fabricated SW3.

Figures 44-a and 44-b show the variation of the microwave index and impedance in SW0 for values of $\mathrm{w}_{2}$ varying from $10 \mu \mathrm{m}$ to $100 \mu \mathrm{m}$, and the regular CPW (Figure 37-a), represented at $\mathrm{w}_{2}=0 \mu \mathrm{m}$. It can be seen that there is a good agreement with simulations. Figure 44-c shows the measured microwave index for the regular CPW, SW0, SW2 and SW3. These experimental results are also in very good agreement with the previously obtained simulation results. The microwave index is improved from 2.36, 3.7 and 6.9 for the regular CPW, SW0 and SW2, respectively, up to 11.6 for SW3. An inset of Figure 44-c shows an optical microscope image of the fabricated SW3. Additionally, Figure 44-d depicts the frequency response measured for a range up to $40 \mathrm{GHz}$.

With the aim of completing the characterization of the coplanar waveguides Figure 45 shows the simulated and measured losses for the slow-wave CPW. 
Figure 45-a shows the losses corresponding to SW0 as a function of $\mathrm{w}_{2}$ meanwhile Figure 45-b shows the propagation losses for Regular, SW0, SW2 and SW3. A mismatch of $0.5 \mathrm{~dB}$ can be seen in Figure 45-a due to the higher metal thickness of the fabricated SW-CPW $(1 \mu \mathrm{m})$ compared to the simulated $(0.75 \mu \mathrm{m})$. However, a good agreement has been accomplished between simulations and measurements (except for SW2) as it can be seen in Figure 45-b.
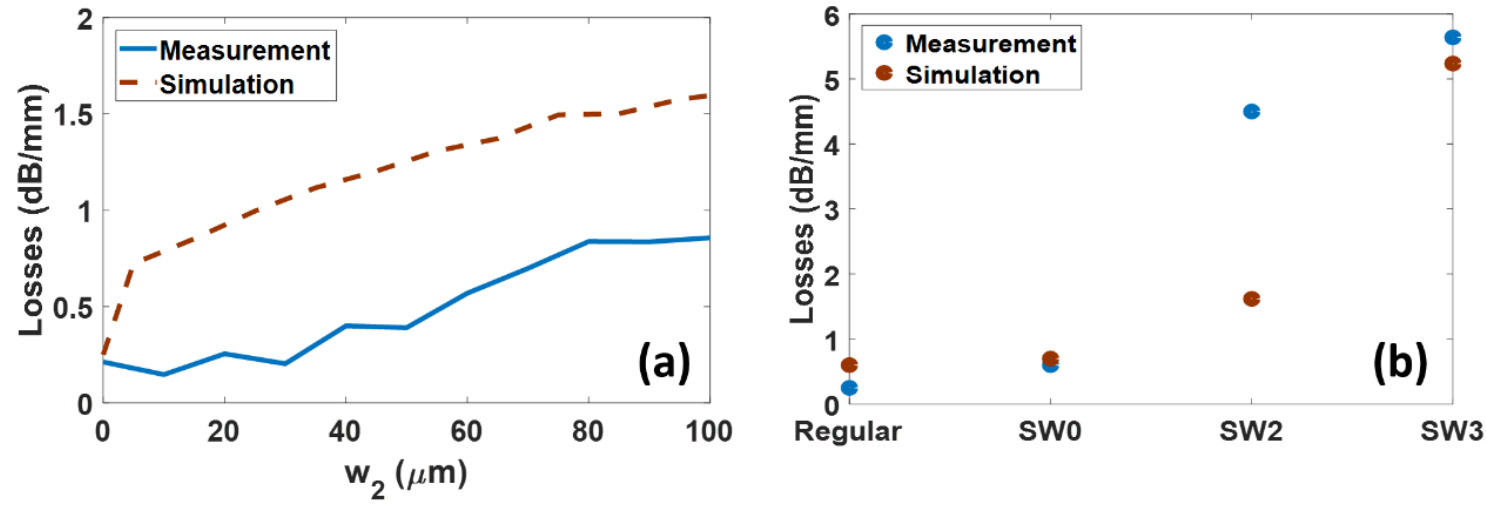

Figure 45. (a) Measured and simulated losses for SW0 as a function of w2 and (b) measured losses for

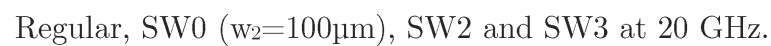

\begin{tabular}{|c|c|c|c|c|c|}
\hline Reference & Year & Structure & $\mathrm{N} \mu$ & $\alpha(\mathrm{dB} / \mathrm{mm})$ & $\mathrm{Z}_{0}(\Omega)$ \\
\hline$[10]$ & 1994 & CPW & 3.15 & 40 & 50 \\
\hline$[11]$ & 2017 & CPW & 3.9 & 2 & 50 \\
\hline$[12]$ & 1996 & CPW & 2.6 & 46 & 50 \\
\hline$[54]$ & 2005 & CPW & 4.47 & $4-6.9$ & 50 \\
\hline$[58]$ & 2013 & CPW & 5.3 & 0.7 & 35 \\
\hline$[67]$ & 2011 & CPW & 4 & 33 & 50 \\
\hline$[69]$ & 1995 & CPW & 3.37 & 2 & 45 \\
\hline$[70]$ & 2009 & CPW & 5.9 & 0.4 & 43 \\
\hline$[71]$ & 2015 & CPW & 7.38 & 1.4 & 34 \\
\hline This thesis (CPW) & 2018 & CPW & 2.5 & 0.25 & 50 \\
\hline This thesis (SW0) & 2018 & CPW & 3.7 & 0.8 & 80 \\
\hline This thesis (SW3) & 2018 & CPW & 11.6 & 5.4 & 50 \\
\hline
\end{tabular}

Table 5. Microwave index, propagation losses and impedance comparison for reported slow-wave transmission lines. 
Table 5 compares the obtained results in terms of microwave index, propagation losses and impedance with the values reported in the last two decades for slowwave CPWs.

The microwave index is largely increased for SW3 with respect to previous works. Furthermore, though propagation losses increase for SW3 with respect to SW0 and the regular CPW, the value remains comparable and even lower than the ones achieved in CPWs with an impedance of around $50 \Omega$.

\subsection{Conclusions}

In summary, we have demonstrated a design approach to increase the microwave index in CPWs. The proposed approach is based on the design of periodically distributed inductive and capacitive elements. A microwave index of 4.7 has been achieved by increasing the inductive behavior of the CPW with small slots on the ground and signal planes. In addition, the effect on the impedance has also been considered and it has been shown that it is possible to achieve high microwave indices while keeping the impedance around $50 \Omega$. Therefore, through the combination of inductive and capacitive elements, a microwave index up to 11.6 has been demonstrated. To the best of our knowledge, such value is the largest to date obtained in planar transmission lines. 


\section{Chapter 4}

\section{RF electrodes design for novel BTO-Si electro-optic modulators}

\subsection{Introduction and State-of-the-Art}

Optical modulators devices are key building-blocks for high speed signal communication and information processing. Such devices have been widely pursued in silicon photonics by means of the plasma dispersion effect [14]. However, the modulation speed is limited by the carrier lifetime and optical losses are also induced. On the other hand, the Pockels effect is intrinsically lossless and ultra-fast but is missing in silicon. In this way, the integration of ferroelectric materials on silicon has opened a new path towards the development of electrooptic modulators based on the Pockels effect. Among these materials, barium titanate $\left(\mathrm{BaTiO}_{3}\right.$ or $\left.\mathrm{BTO}\right)$ is particularly suitable due to its attractive dielectric and electro-optic properties.

EO modulation by means of the Pockels effect allows achieving high modulation speeds without penalizing insertion losses and it has been demonstrated by breaking the crystal symmetry of silicon [77]. In such a manner, interesting results have been obtained through depositing highly stressed nitride layers over silicon [78], [79]. However, there is still need to study the influence of carrier effects and one order of magnitude lower effective Pockels coefficient has been measured at 
high frequencies [80]. The integration of ferroelectric materials on silicon has therefore become a promising way to achieve EO modulation via Pockels effect. $\mathrm{BaTiO}_{3}$ is one of the best candidates because of their large Pockels coefficient $\left(\mathrm{BaTiO}_{3}\right.$ presents a larger Pockels coefficient compared to lithium niobate). BaTiO3 modulators have been mainly investigated so far on top of magnesium oxide $(\mathrm{MgO})$ substrates [81]-[87]. However, the potential of growing high-quality thin film crystalline layers of BTO on strontium titanate $\left(\mathrm{SrTiO}_{3}\right)$ templated silicon and silicon on insulator (SOl) substrates [88]-[90] has recently opened a path towards the development of hybrid BTO silicon EO modulators with disruptive performance [91]-[95].

On the other hand, ferroelectric materials with high permittivity have been largely investigated for ceramic applications [96], [97]. However, these materials have also found application in other fields such as phased array antennas [98] or planar microwave integrated phase shifter [99]. In this context, barium titanate $\left(\mathrm{BaTiO}_{3}\right.$ or BTO) is one of the most representative ferroelectric materials. For photonic applications, as it has been previously mentioned, BTO is one of the best candidates to develop high performance electro-optical modulators thanks to its large Pockels coefficients [100]. The Pockels coefficients in bulk BTO crystals is several orders of magnitude higher than lithium niobate $\left(\mathrm{LiNbO}_{3}\right)$, which has motivated the development of BTO-based photonic devices. In this way, high modulation efficiencies and the potential for achieving modulation bandwidths above $40 \mathrm{GHz}$ have been demonstrated by using BTO deposited on top of magnesium oxide (MgO) substrates [81], [101]-[104]. However, in the last years, the successful growth of high quality BTO films on silicon has opened a new path for providing electro-optic functionalities to the silicon photonic platform [88], [90], [94], [105] and [106]. The possibility of an integration process compatible with large size silicon wafers makes BTO more attractive than other materials such as lithium niobate in which the integration is based on bonding processes [107].

The electro-optical performance of hybrid BTO/Si modulators has been mostly analyzed under static conditions and simulated modulation efficiencies as low as $0.27 \mathrm{~V} \cdot \mathrm{cm}$ have been reported [93], [95], [108], [109]. However, high speed modulation cannot be achieved unless RF electrodes are properly designed. Hence, an accurate measurement of the relative permittivity of the BTO layer is a prerequisite to accomplish such design task. 
The BTO permittivity depends on the crystallographic axes of the material. A value of about 56 for the direction parallel to the optical axis and of about 2200 for the direction perpendicular to the optical axis have been taken as a gauge of good quality of clamped, single-domain crystals [100]. However, the permittivity may show a huge variability depending on the fabrication conditions. Low values between 83 and 120 have been reported for films deposited at room temperature by RF sputtering [110]. Conversely, high values up to 6000 have also been measured and correlated with the grain size and microstructural characteristics of the material [98]. The permittivity may also depend on the thickness of the BTO layer and the frequency of the applied electric field. Values between 230 and 1000 have been measured for BTO films with thicknesses ranging from 30 to 580 $\mathrm{nm}$ at a low frequency of $1 \mathrm{KHz}$ [111]. At higher frequencies, a decrease of permittivity from 2200 to 500 has been estimated for RF frequencies among 1 and $40 \mathrm{GHz}[112]$.

In this chapter, the relative permittivity of thin BTO films deposited on siliconon-insulator (SOI) substrates is first analyzed. A multiline method is proposed to estimate the effective permittivity at wafer level and then electromagnetic simulations are carried out to extract the BTO permittivity. Therefore, the BTO can be characterized in the same growth conditions as the ones used to fabricate the devices. Once the BTO permittivity is obtained, the electrodes have been designed, fabricated and characterized as well as hybrid BTO/Si electro-optic modulators.

\subsection{Relative permittivity extraction of Barium Titanate}

The relative permittivity of the BTO has been characterized on the same multilayer stack, shown in Figure 46, used to develop the optimum travellingwave electrodes for high speed modulation. In this case, the BTO permittivity cannot be measured as in a conventional capacitor by extracting the capacitance between its plates [96]. Therefore, a multi-line method can be used to estimate the effective permittivity of the complete multilayer stack. The proposed multiline method is described in Chapter 3, section 3.3.2. 


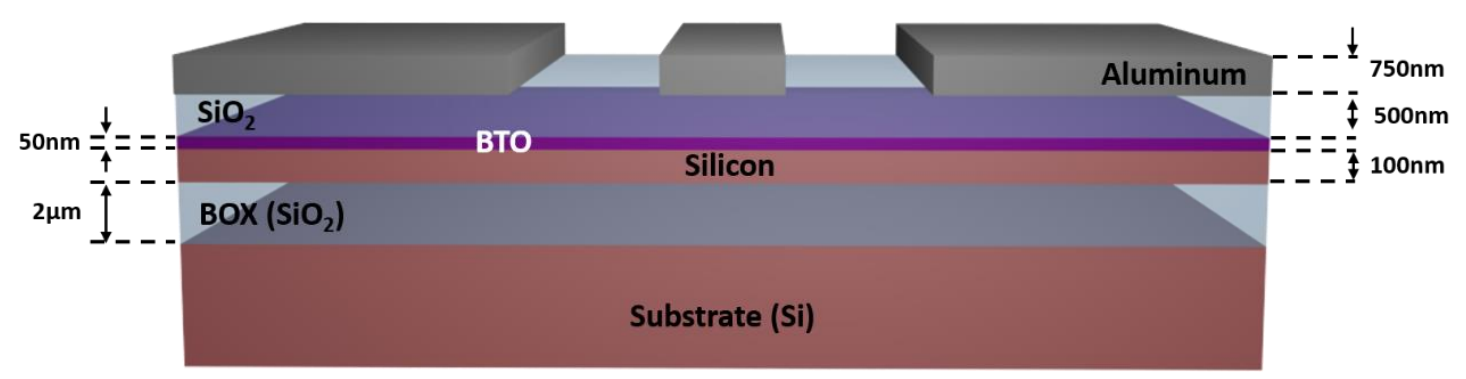

Figure 46.RF coplanar waveguide structure used to characterize the BTO permittivity.

BTO layers have been grown on a SOI substrate with a device silicon layer thickness of $100 \mathrm{~nm}$ and a buried oxide thickness of $2 \mu \mathrm{m}$. A thin (3-4 nm thick) $\mathrm{SrTiO}_{3}$ buffer is required to accommodate the crystallographic and chemical heterogeneity between BTO and Si and ensure single crystallinity and high crystal quality of the BTO layer. Molecular beam epitaxial (MBE) was used to grow the $\mathrm{SrTiO}_{3}$ template, as described in [88]. Then, two different approaches, MBE and RF sputtering, were employed to grow the BTO layer with a thickness of about $50 \mathrm{~nm}$.
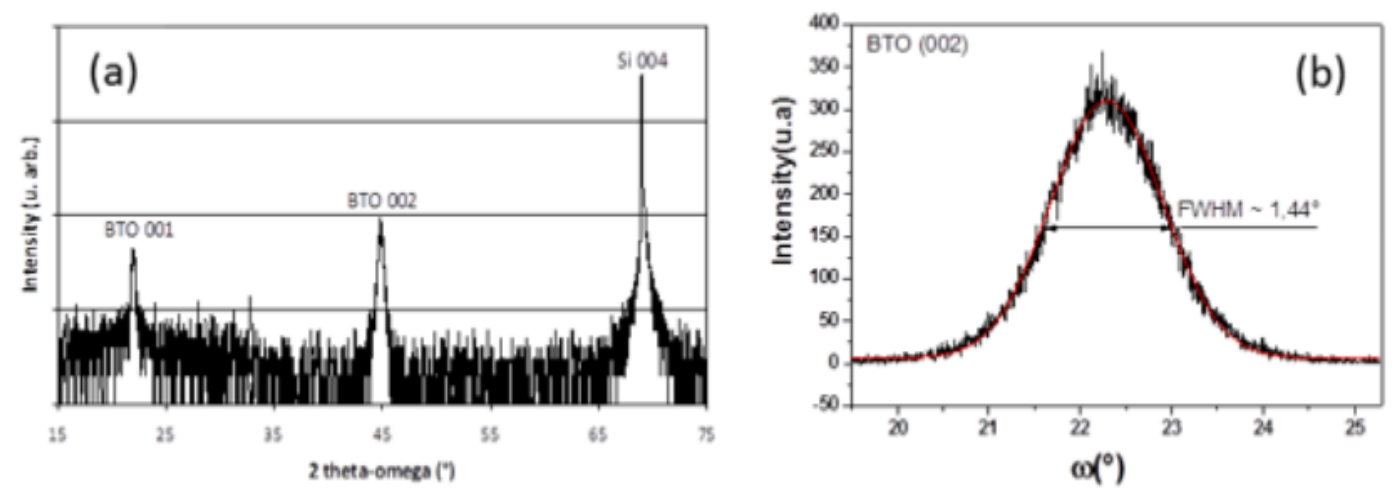

Figure 47. (a) XRD pattern of 50nm-thick $\mathrm{BaTiO}_{3}$ film grown on $\mathrm{SrTiO}_{3}-\mathrm{SOI}$ template by RF-sputtering.

(b) Corresponding rocking curve: the full width at half maximum is $1.44^{\circ}$. (images courtesy of INL).

RF-sputtering was used to grow 50 nm-thick BTO film on the $\mathrm{SrTiO}_{3} / \mathrm{SOI}$ template. A BTO stoichiometric target was sputtered under $\mathrm{Ar}$ and $\mathrm{O}_{2}$ mixing atmosphere and using a RF generator at a substrate temperature of $650^{\circ} \mathrm{C}$. After growth, the BTO layer was submitted to rapid thermal annealing to improve its structural quality and ensure good oxidation of the thin layer. X-ray diffraction (XRD) pattern and corresponding rocking curve are shown on the Figure 47. On the other hand, using a similar $\mathrm{SrTiO}_{3} / \mathrm{Si}$ template, a $50 \mathrm{~nm}$ thick BTO was grown by $\mathrm{MBE}$ using $\mathrm{Ba}$ and $\mathrm{Ti}$ co-deposited at $600^{\circ} \mathrm{C}$ under an oxygen partial pressure of about $10^{-5}$ Torr. More details can be found in [88]. Figure 48 shows the corresponding XRD pattern with a magnified section which shows c-axis orientation for MBE grown BTO. 
(a)

(b)

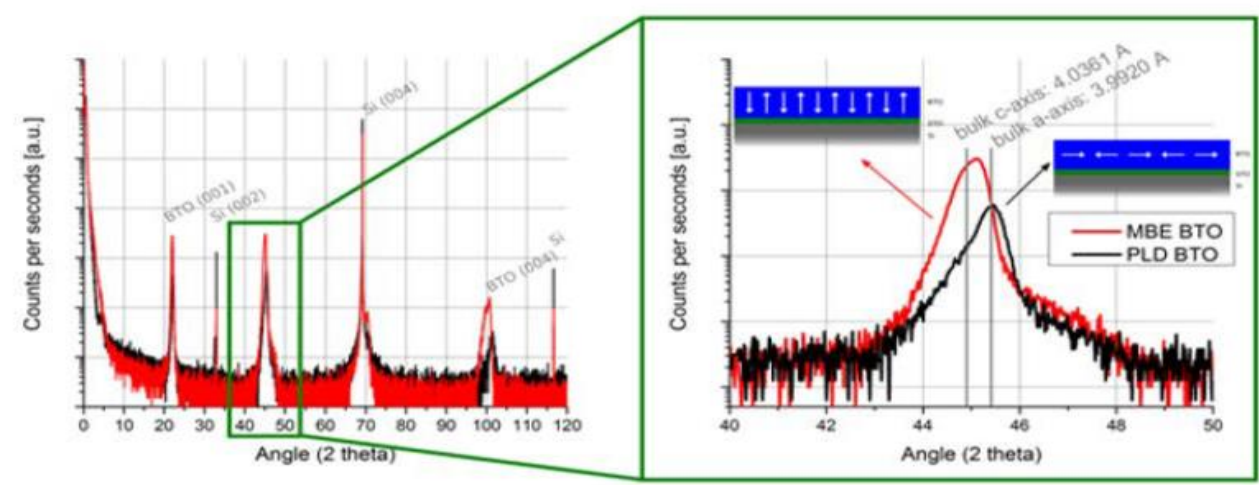

Figure 48. X-ray diffraction pattern of BTO films: 25nm BTO grown by MBE (red curve) and 60nm BTO grown by PLD (black curve). (a) XRD diffraction pattern of BTO on STO on Si with epitaxial growth of the oxide on the substrate. (b) Magnified section of (a) to show the [002] BTO diffraction peak. Illustration of the c-axis and a -axis orientations in inset (images courtesy of IBM).

RF coplanar waveguides (CPW) were fabricated on top a $500 \mathrm{~nm}$-thick $\mathrm{SiO}_{2}$ layer deposited by PECVD on the BTO/SOI substrate. The CPWs have a gap of 45 $\mu \mathrm{m}$ and a width of $45 \mu \mathrm{m}$ for the central line. The metal is aluminum with a thickness of $750 \mathrm{~nm}$. Transmission lines with different lengths were used to apply the multiline method. A length of $1 \mathrm{~mm}$ was chosen for the thru (reference) line while lengths of $2 \mathrm{~mm}, 2.5 \mathrm{~mm}$ and $3 \mathrm{~mm}$ were used for the other lines (shown in Figure 49). The difference in length between the thru and the rest of the lines is acceptable for minimizing the inaccuracy caused by the manual alignment of RF probes on the metal pads. The scattering parameters of the CPW were measured and then used to extract the effective permittivity.

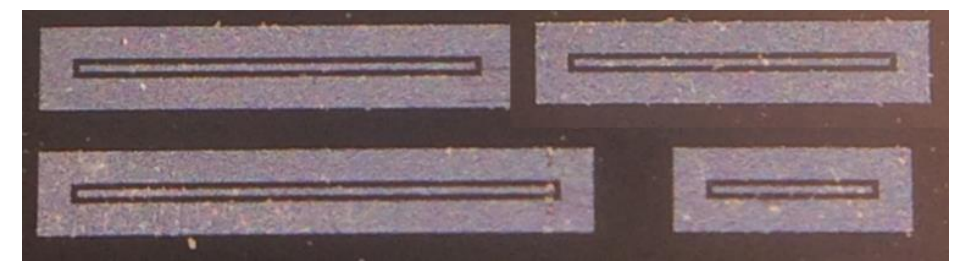

Figure 49. Fabricated CPWs with lengths between $1 \mathrm{~mm}$ and $3 \mathrm{~mm}$.

The effective permittivity acquired from the characterization measurements has been compared with the obtained from the simulations to match both effective permittivities and, by cross-checking the results, estimate the value of the BTO permittivity. Figure 50-a shows the schematic of the measurement set-up and Figure 50-b shows the real part of the effective permittivity as a function of the frequency for the fabricated CPWs. The effective permittivity obtained is also very similar for the samples with BTO fabricated by MBE or RF sputtering, as it can be seen in Figure 50-b. 

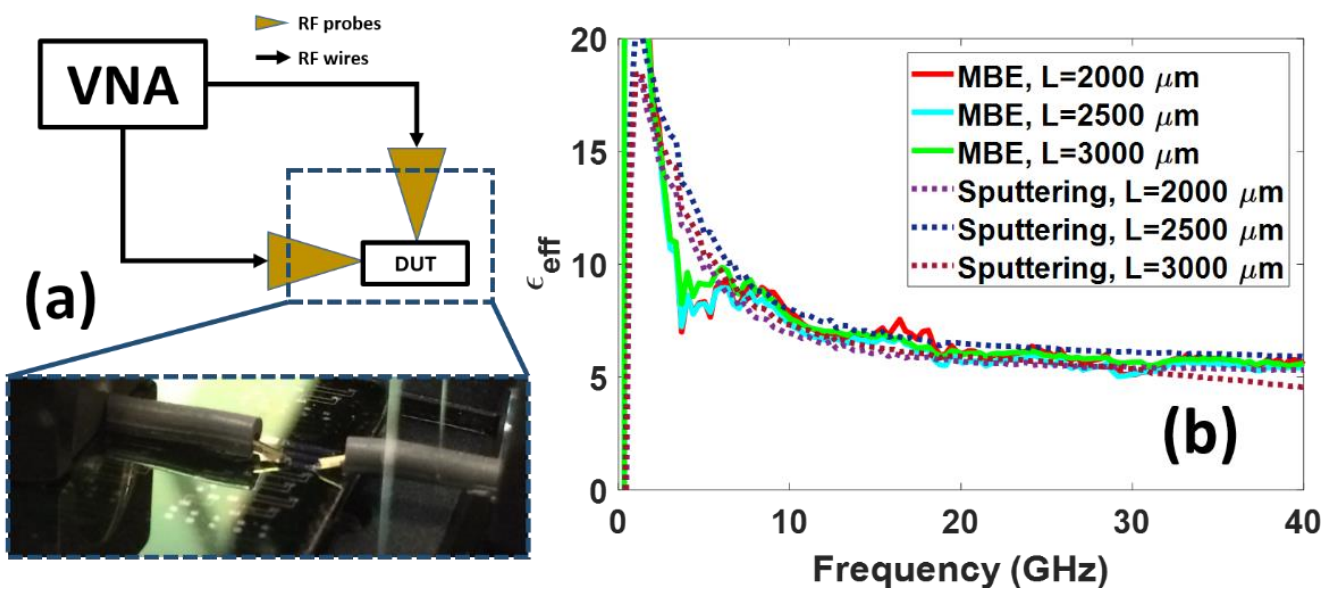

Figure 50. (a) Schematic of the measurement set-up by using a vector network analyzer (VNA) and photo inset zoom of the (DUT) area. (b) Measured effective permittivity as a function of the frequency for the samples with BTO fabricated by MBE and RF sputtering
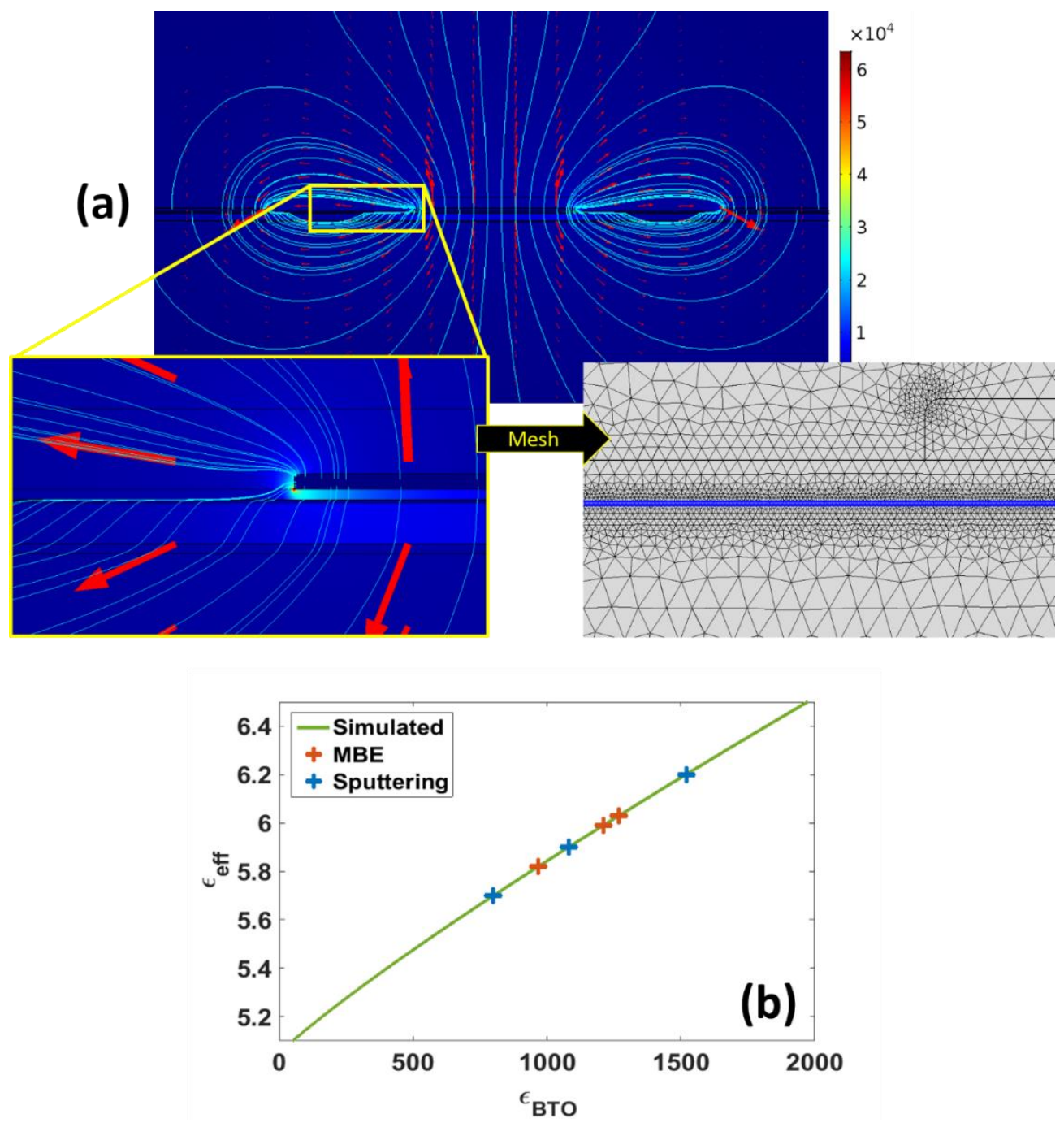

Figure 51. (a) Simulated electric field, distribution and flux lines of the quasi-TEM mode at the frequency of $20 \mathrm{GHz}$. The inset shows with more detail the CPW structure and the properly generated mesh for the BTO thin film and the whole structure, and (b) simulated effective permittivity of the CPW as a function of the BTO relative permittivity (green line) and measured values extracted at $20 \mathrm{GHz}$ from the different delay lines fabricated in the samples with BTO grown by MBE and RF sputtering. 
Simulations have been carried out with COMSOL Multiphysics. Figure 51-a shows the electric field distribution of the quasi-TEM mode at the frequency of $20 \mathrm{GHz}$ and the fine mesh used to properly run the simulations. COMSOL Multiphysics results provides the microwave index which is directly related to the effective permittivity, as it has been previously shown in Equation (3.32).

The relative permittivity of the BTO layer has been modified step by step from 100 to 2000 in the simulations in order to match the real part of the effective permittivity with the measured values. Figure 51-b shows the obtained simulation results for $20 \mathrm{GHz}$ (green line) and the measured values extracted from the different delay lines for both BTO fabrication processes. The BTO layer has a relatively small influence on the effective permittivity due to its low thickness. Simulation and experimental values are matched for a BTO permittivity between 800 and 1600. Hence, an average value of 1200 has been chosen to design the optimum electrodes for high speed modulation. The high value obtained for the BTO is in agreement with BTO grown mostly with c-axis ferroelectric domain orientation [100], [112], which was previously confirmed by X-ray diffraction (XRD) measurements (Figure 47 and Figure 48).

Nevertheless, there is an obvious variation on the obtained BTO permittivity. The measured variation in the effective permittivity is around $5 \%$ of its average value. However, this relatively small variation is translated into a higher variation of the estimated BTO relative permittivity mainly because the influence of misalignments of the RF probes with the coplanar waveguides and the thin thickness of the BTO layer. In this way, Figure 52-a shows the simulated effective permittivity for different thicknesses of the BTO layer as a function of the BTO permittivity. In this way, Figure 52-b shows the effective permittivity with and without $\mathrm{SiO}_{2}$ cladding. Assuming the same influence of the misalignment error, with a BTO thickness of 300nm the obtained BTO permittivity should be more accurate result. However, the extraction of the BTO permittivity on the samples used for the modulator fabrication is in this case a requirement. 

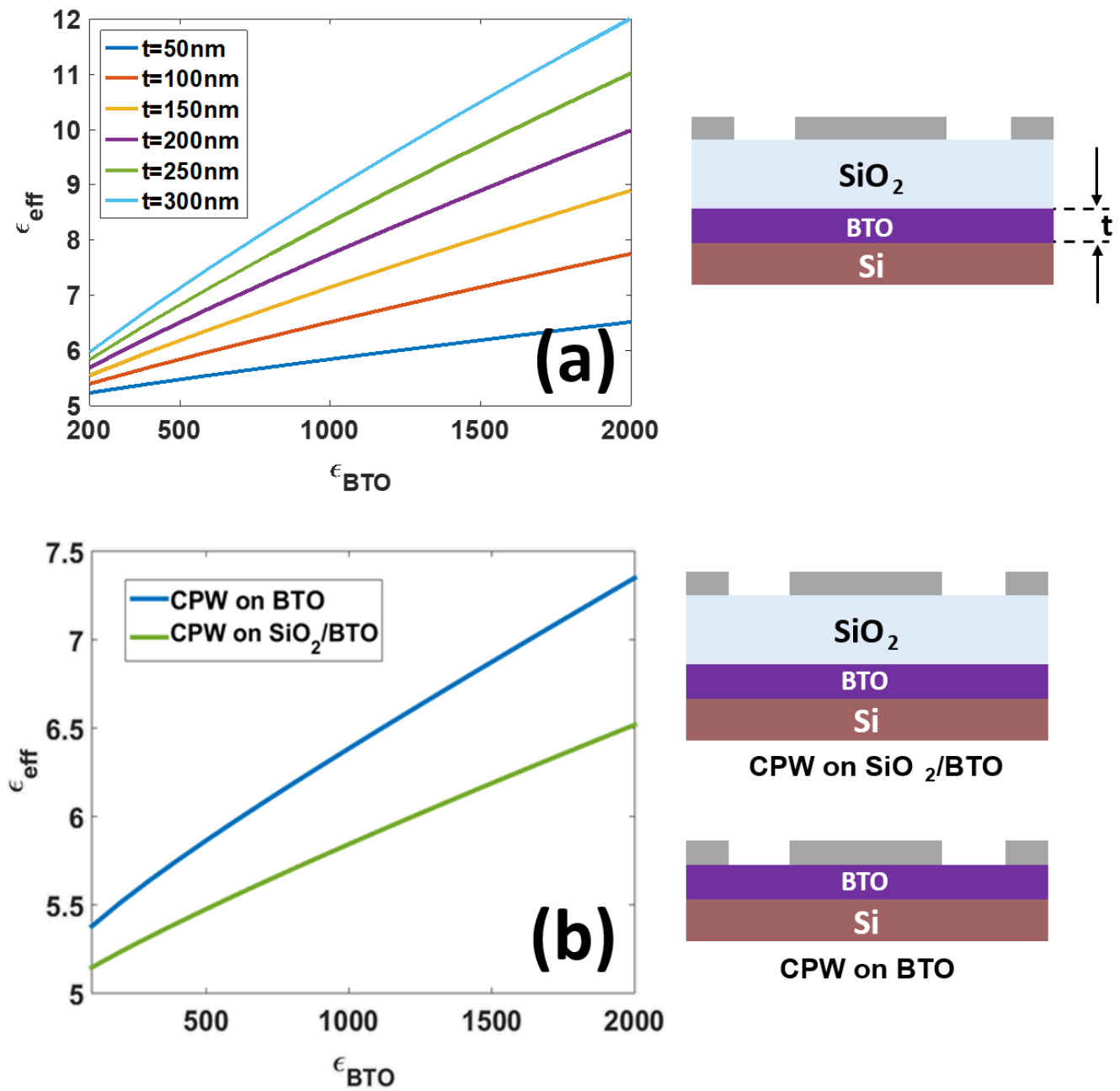

$\mathrm{CPW}$ on $\mathrm{SiO}_{2} / \mathrm{BTO}$

Figure 52. (a) Variation of effective permittivity as a function of the BTO permittivity for both possible configuration, with the CPW over the SiO2 cladding and directly over the BTO, and (b) effective permittivity as a function of the BTO permittivity for different BTO layer thicknesses.

Furthermore, it should also be noticed that the major component of the electric field in-between the electrodes is mainly in the horizontal direction. Therefore, in this case, the electric field lines are more concentrated in the BTO (magnified section of the Figure 51-a) if the permittivity is much higher than the ones from the surrounding materials, which is desirable to improve the modulation efficiency. Additionally, a CPW has been simulated without the silica cladding between the BTO layer and the metallic strips of the coplanar waveguide, and there is not a significant difference with the obtained effective permittivity between with or without silica cladding. Therefore, the design of the electrooptical modulator can be addressed with the obtained results. 


\subsection{Electrode and electro-optical design of BTO-Si based modulator}

The hybrid BTO/Si optical waveguide depicted in Figure 53-a has been proposed for enabling electro-optical modulation with high performance. A layer of amorphous silicon (a-Si) is deposited on top of the BTO/SOI structure and then etched down to form the optical waveguide. The influence of the waveguide parameters on the static electro-optical performance has been previously reported [93]. The electrodes are placed on top of the BTO layer after opening lateral windows in the $\mathrm{SiO}_{2}$ cladding to enhance the modulation efficiency.

The high value of the BTO relative permittivity $\left(\varepsilon_{\mathrm{r}} \sim 1200\right)$ will increase the capacitance of the electrodes and thus the impedance will be reduced with respect to other materials with lower dielectric constants. A narrow gap between electrodes and the optical waveguide is desirable to improve the modulation efficiency though a too small value will give rise to optical losses due to interaction of the optical mode with the metal electrodes [93]. Coplanar strip-line (CPS) electrodes have been designed to achieve the best modulation performance in a travelling-wave configuration.

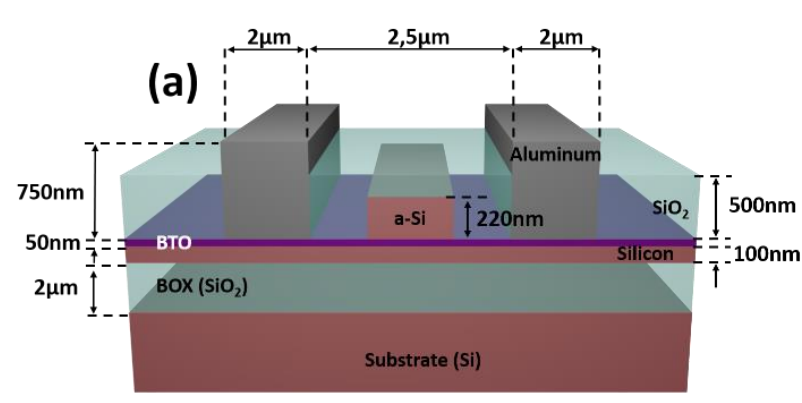

(c)

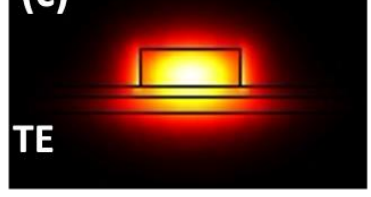

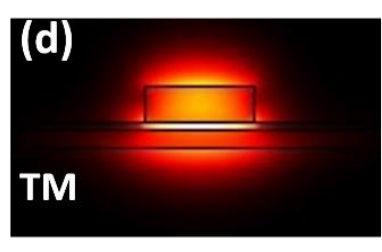
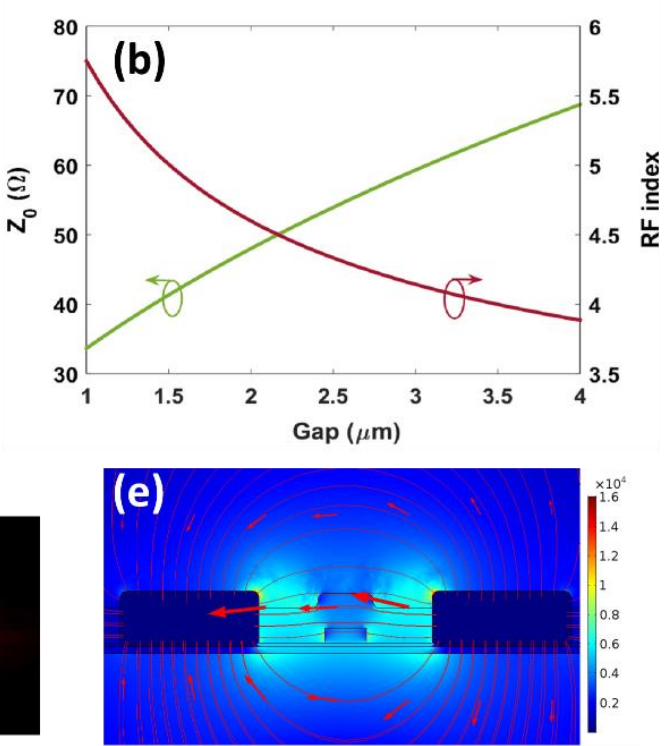

Figure 53. (a) Hybrid BTO/Si optical waveguide with coplanar strip-line electrodes and (b) simulated impedance of the CPS electrode and microwave index of the RF mode at $20 \mathrm{GHz}$ as a function of the gap and for an electrodes width of $2 \mu \mathrm{m}$, (c) optical mode profile for TE and (d) TM polarizations, and (e) simulated electrical field for the CPS electrode at a frequency of $20 \mathrm{GHz}$. 
The maximum thickness of the metal electrodes allowed by the fabrication process, which is around $750 \mathrm{~nm}$ as depicted in Figure 53-a, has been initially fixed. The gap and width of the CPW electrode has then been designed taking into account their influence on the impedance and the microwave index. Both fundamental parameters, the impedance and the microwave index, decrease when the electrode width increases. However, they change in an opposite way when the gap is varied, as it is shown in Figure 53-b. The microwave and optical group indices should be matched to maximize the modulator bandwidth. The simulated optical group index of the hybrid BTO/Si optical waveguide is 3.66 for TE polarization (Figure 53-c) and 3.85 for TM polarization (Figure 53-d).

Thereby, the optimum parameters of the CPS electrode have been found for a gap of $2.5 \mu \mathrm{m}$ and an electrodes width of $2 \mu \mathrm{m}$. The resulting impedance and microwave index are $54 \Omega$ and 4.52, respectively, as it can be observed in Figure 53-b. An impedance close to $50 \Omega$ allows achieving maximum voltage transfer and avoids electrical reflections, which is desirable to maximize the modulator performance. In addition, the potential bandwidth by assuming negligible microwave propagation losses $(\alpha=0)$ can be easily estimated by:

$$
\Delta f=\frac{1.89 c_{0}}{\pi\left(n_{\mu}-n_{o}\right) L}
$$

where $c$ is the speed of light, $L$ is the modulation length and $n_{\mu}$ and $n_{o}$ are the microwave and optical group indices [9]. Bandwidths higher than $40 \mathrm{GHz}$ for modulation lengths up to $5.5 \mathrm{~mm}$ would be feasible based on Equation (4.1). The designed CPS electrode has been fabricated and characterized. The experimental impedance was extracted from the reflection coefficient of the scattering parameters. Figure 54-a shows the simulated and experimental impedance and Figures 54-c and 54-d show the scattering parameters obtained for both fabricated BTO layers as a function of the RF frequency. It can be seen that the influence of the BTO fabrication process on the experimental impedance values is low. Furthermore, there is a rather good agreement between simulation and experimental results. 

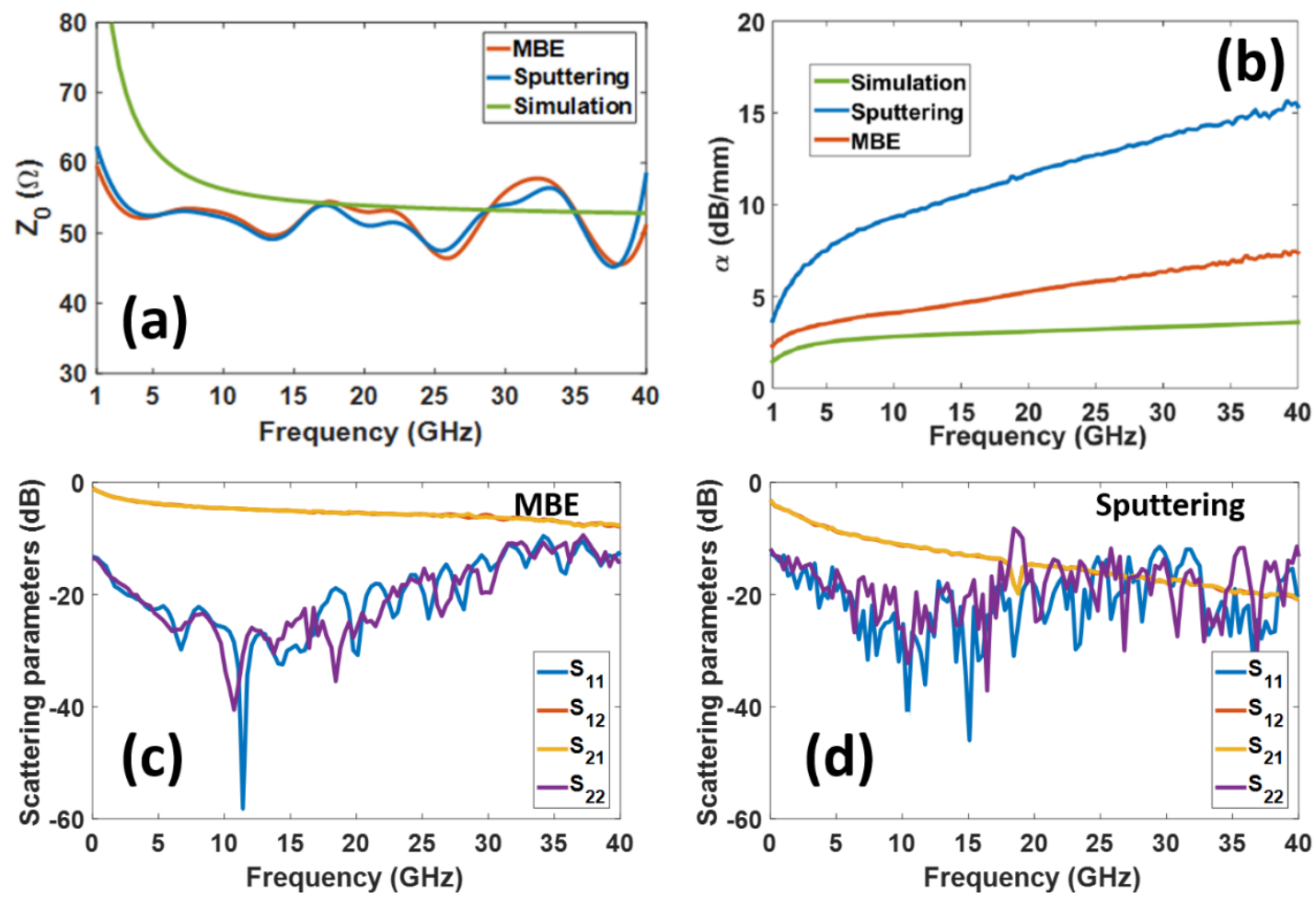

Figure 54. Simulated and experimental (a) impedance and (b) microwave propagation losses of the designed CPS electrode as a function of the RF frequency. Only conductor losses are included in the simulations. (c) and (d) show the experimentally measured scattering parameters of CPS electrodes for a length of $500 \mu \mathrm{m}$ for both fabrication methods, MBE and RF sputtering respectively.

The microwave propagation losses have also been extracted from the measured scattering parameters. Propagation losses are mainly originated due to conductor and dielectric losses [52]. Conductor losses have a square root dependence with frequency due to the skin effect. Besides, dielectric losses depend on the polarization and ohmic losses of the dielectric materials. At high frequencies, the heating associated with the polarization become the dominant source of losses. Figure 54-b shows the experimental losses as a function of the RF frequency. Simulations considering only the conductor losses have also been carried out to compare with experimental results. The difference between experimental and simulation results increases for high RF frequencies. Such higher losses are attributed to dielectric losses of the materials but also from additional conductor losses due to surface roughness at the electrodes. However, it is interesting to notice that the sample with BTO fabricated by sputtering has higher losses than the sample with BTO growth by MBE. Therefore, the obtained results also suggest that BTO losses may play an important role and that lower losses are achieved by MBE. 
Furthermore, for a given microwave propagation losses and considering that the CPS electrode is terminated by its impedance, the small signal electro-optical modulation response can be calculated by:

$$
T_{E O}(f)=e^{-\frac{\alpha L}{2}} \sqrt{\frac{\sinh ^{2}\left(\frac{\alpha L}{2}\right)+\sin ^{2}\left(\frac{2}{\Delta f}\right)}{\left(\frac{\alpha L}{2}\right)^{2}+\left(\frac{2}{\Delta f}\right)^{2}}}
$$

where L is the modulation length, $\alpha$ is the loss coefficient, $\Delta f$ is given by Equation (4.1) and $\mathrm{f}$ is the RF frequency [18].
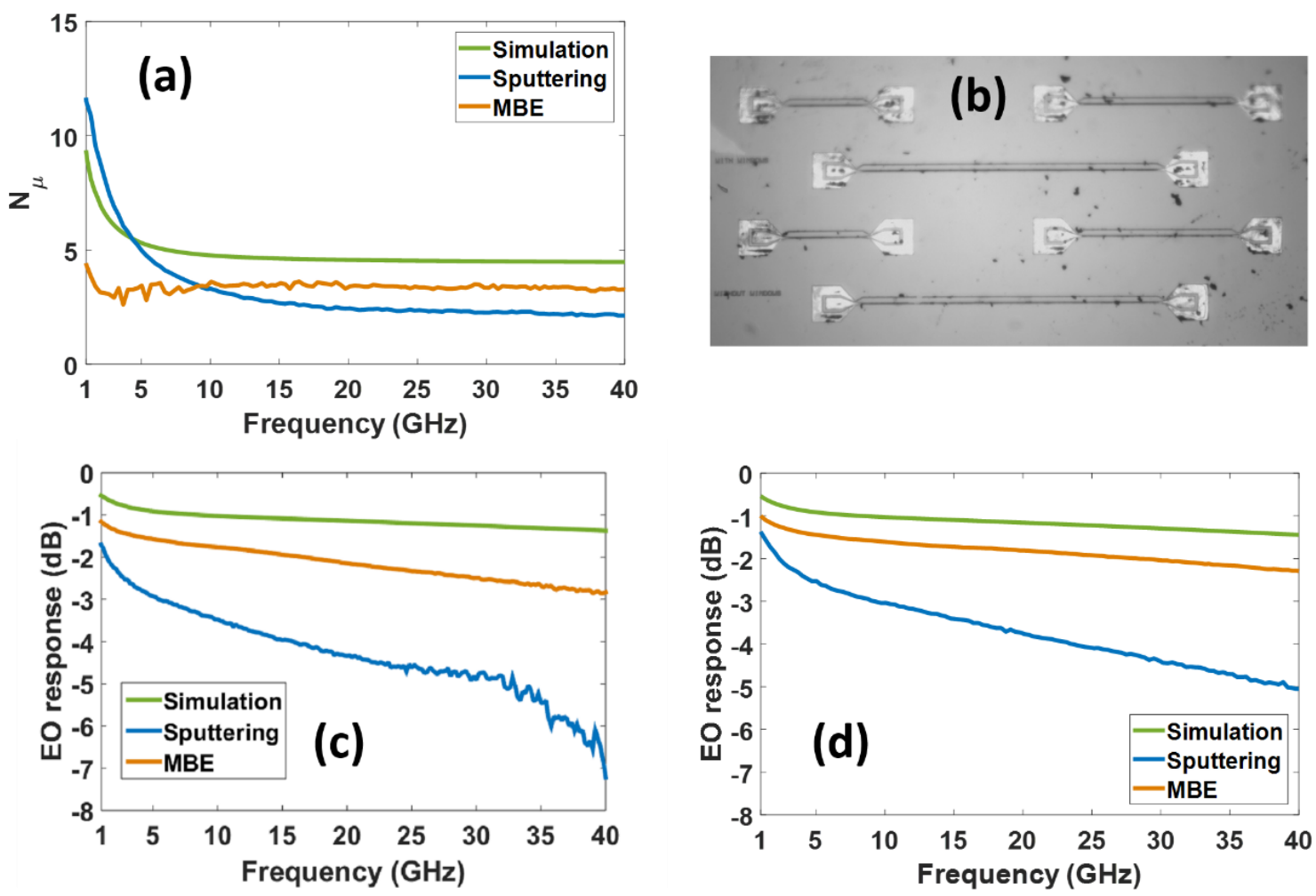

Figure 55. (a) Microwave index measured for the modulator CPS, (b) microscope image of the straight CPS measured electrodes with different lengths, (c) calculated electro-optical response taking into account the simulated microwave index and the measured propagation losses and (d) electro-optical response for measured microwave index and losses. Both electro-optical responses are calculated for a length of $1.5 \mathrm{~mm}$ and TM polarization $\left(\mathrm{n}_{0}=3.85\right)$.

Figure 55-a shows the microwave index obtained for the fabricated CPS that can be observed in Figure 55-b. Hence, Figure 55-c shows the simulated frequency response for a modulation length of $1.5 \mathrm{~mm}$, TM polarization and taking into account the simulated microwave index and both simulated and measured microwave losses (Figure 54-b). The measured microwave index has been taken 
into account in Figure 55-d and as a result, the electro-optical bandwidth has been slightly increased because the measured microwave index is relatively smaller than the simulated.

Furthermore, the electro-optical response is practically the same for TE polarization $\left(\mathrm{n}_{\mathrm{o}}=3.66\right)$. Therefore, for both light polarizations, the modulation bandwidth would be drastically reduced to around $5 \mathrm{GHz}$ for the BTO grown by sputtering due to the high losses. However, a modulation bandwidth of $40 \mathrm{GHz}$ would be achieved for the BTO grown by MBE. Additionally, a half-wave voltage, $\mathrm{V} \pi$, of around $5 \mathrm{~V}$ for TM polarization and $7.9 \mathrm{~V}$ for TE polarization has been estimated by simulation for c-axis oriented BTO and the modulation length of $1.5 \mathrm{~mm}$.

\subsection{Characterization of BTO-Si based modulator}

Once the modulator design has been carried out, this section delves with the performance characterization of the fabricated EO modulator. Unfortunately, the MZI modulators with BTO grown by sputtering has been not suitable for its experimental characterization because of the higher microwave losses of the CPS over the sputtering grown BTO (shown in Figure $54-\mathrm{b}$ ) and the emergence of some amorphous BTO regions, instead of crystalline BTO, which makes the Pockels effect become weak or even negligible. Figure 56 highlights the amorphous regions in the BTO thin film.

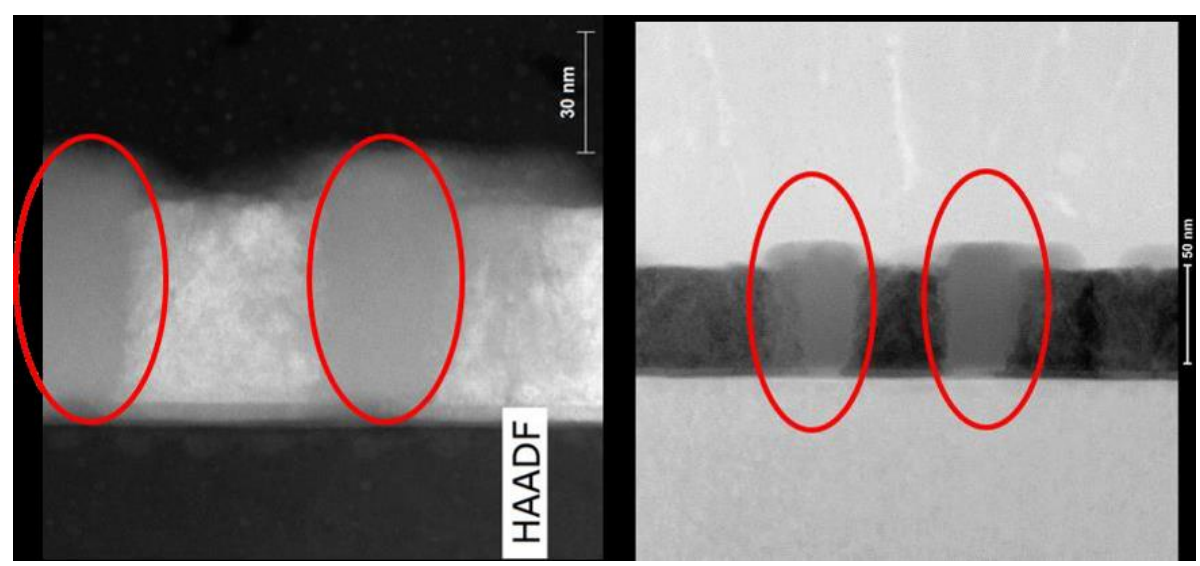

Figure 56. SEM image of some amorphous BTO grown with RF-sputtering [8].

However, the good quality of MBE grown BTO and the low microwave losses measured open the door to the fabrication and the characterization of the BTO- 
Si based modulator using MZI structures. Figure 57-a shows a conceptual image of the fabricated BTO-Si EO modulator, with two metallic strips which represents the previously designed CPS in the active region of the MZI.
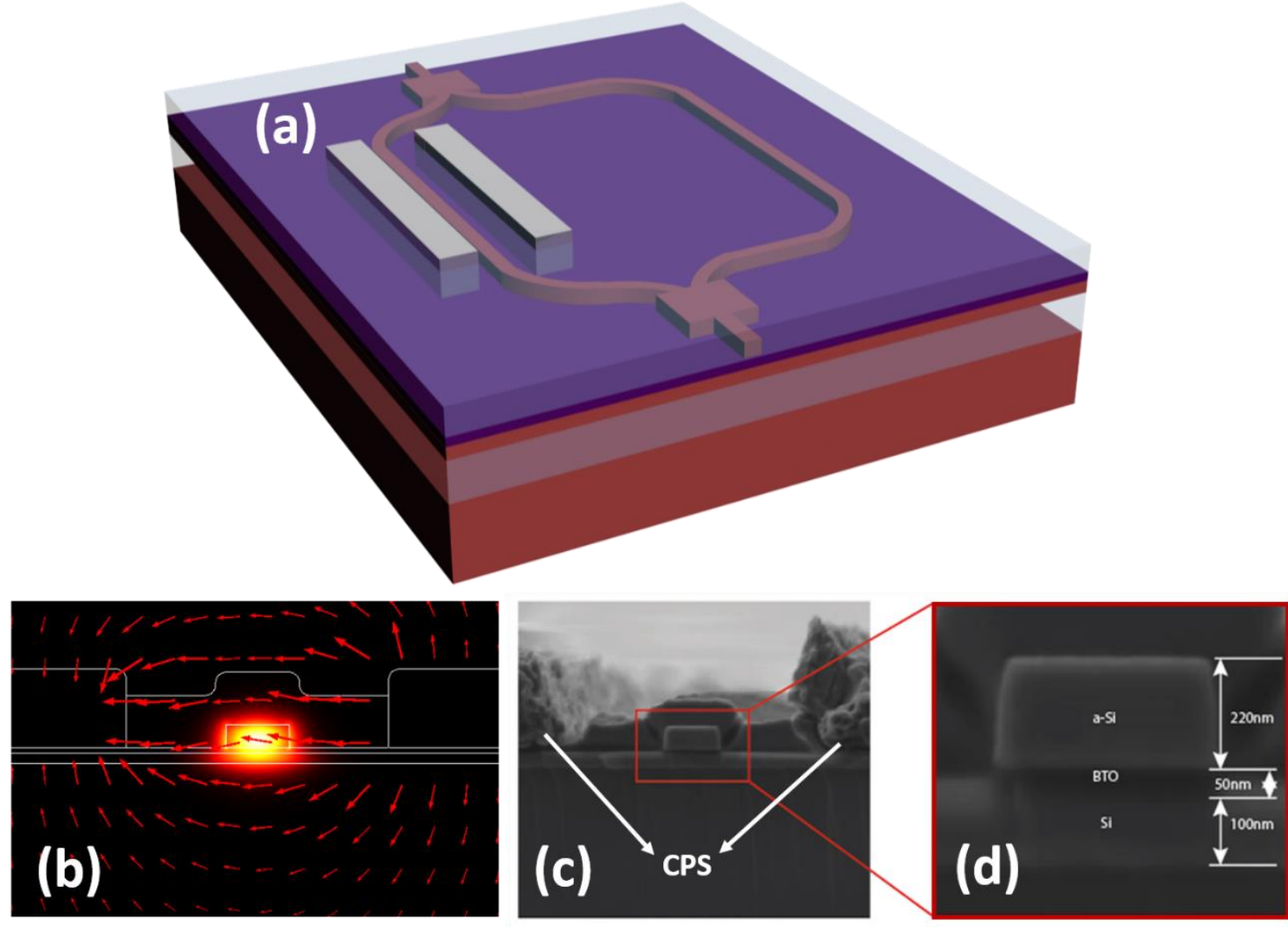

Figure 57. (a) Concept art of the fabricated modulators based on MZI structure, (b) simulation image with both optical and electrical modes, (c) SEM image of a transversal cut in the active region of the MZI and (d) amplified SEM image focused on the optical section.

Hence, Figure 57-b shows the electrical and optical modes (TE) simulated in the active region of the modulator structure. Furthermore, Figure 57-b is very useful to check the corresponding fabricated structure shown in the SEM picture of the Figure 57-c. Additionally, Figure 57-d shows an amplified image of the optical region shown in Figure 57-c, with the main material configuration and dimensions highlighted.

\subsubsection{Electro-optical set-up and final results}

In order to properly characterize the BTO-Si modulator, the used experimental set-up is shown in Figure 58. Analogic and digital characterization have been carried out to evaluate the performance of the modulator. For digital characterization it is required a bit patter generator which uses an external RF generator as an external clock and provides a non-return-to-zero pseudorandom 
bit sequence with a length of $2^{7}-1$ bits. The bit pattern generator is controlled by a RF generator serving as an external clock. The output of the bit pattern generator is amplified with an RF amplifier. Then, through a bias-tee, it is connected to RF probes which are in contact with the coplanar waveguides of the modulator. The bias-tee is required to apply a DC voltage to the modulator. Regarding to analogic characterization, the set-up results very similar but the RF generator is used to provide the modulating signal and is directly connected to the RF amplifier.

By the other hand, the continuous wave laser (CW laser) is providing the modulator from an optical input previously managed by the polarization control. The light goes through the device under test (DUT), and therefore, the modulated output is amplified by an EDFA (erbium doped fiber amplifier) and filter in order to eliminate the noise previously introduced by the optical amplifier. Finally, the modulated signal is converted to the electrical domain by a photodiode and evaluated in the digital communications analyzer (DCA), where the eye diagram becomes observable if the modulator performance is good enough.

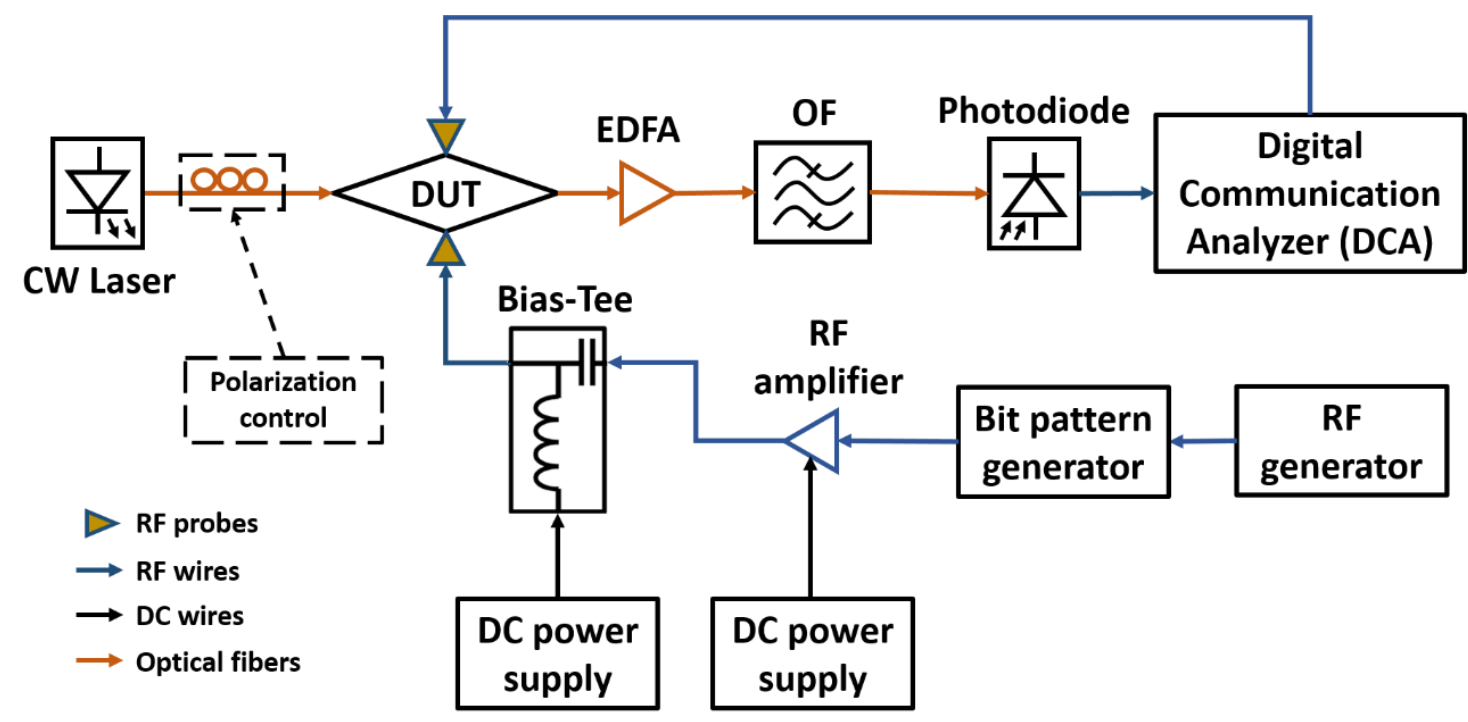

Figure 58. Schematic of the experimental set-up for digital characterization for the BTO-Si modulator

Figure 59 shows pictures of the experimental set-up used to perform the digital characterization of the electro-optical modulator. Additionally, an enlargement picture of the modulator ready to be tested with the RF probes and the optical fibers aligned with the grating coupler has been also added.

The characterization of the modulator has been carried out after the scattering parameter extraction via VNA. Figure 60-a shows the scattering parameters of 
the modulator electrodes (active length of $1 \mathrm{~mm}$ ) with an inset picture of the modulator electrodes with lengths of $0.5,1$ and $2 \mathrm{~mm}$. Despite the insertion losses about $4 \mathrm{~dB}$, the propagation losses are the expected for $1 \mathrm{~mm}$ length modulator. Moreover, the reflection parameters below $10 \mathrm{~dB}$ ensure an impedance close to 50 $\Omega$. Therefore, EO characterization has been accomplished. Additionally, the electro-optical bandwidth is estimated from the electrical response of the modulator electrode [17], [115]. The modulation bandwidth calculated from the $\mathrm{S}_{21}$ at $-6 \mathrm{~dB}$ is about $18 \mathrm{GHz}$.

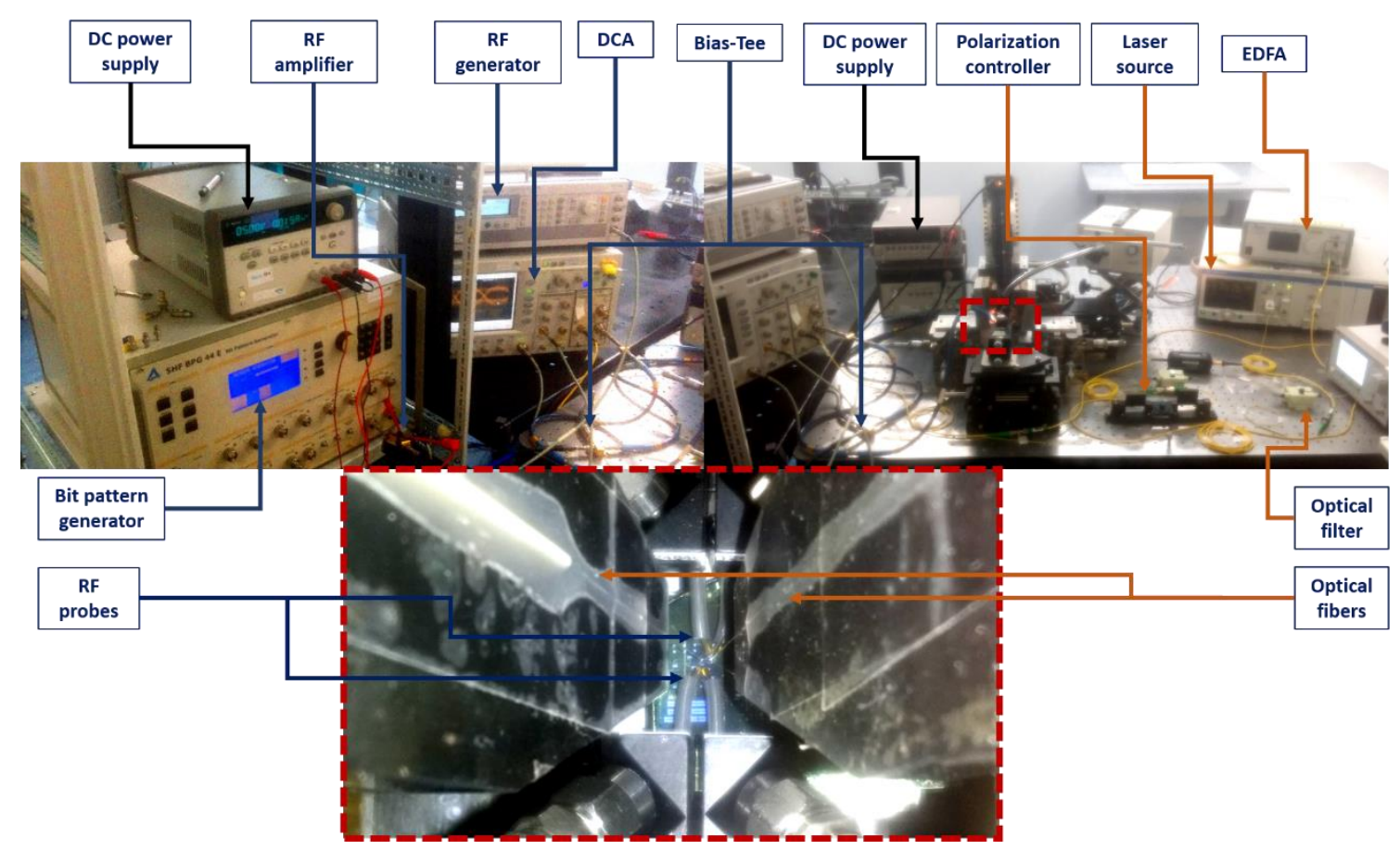

Figure 59. Digital modulation experimental set-up pictures.

The obtained EO bandwidth is lower than the previously calculated shown in Figure 55. The reduction can be basically attributed to the increase of the microwave losses. The calculated losses in Figure 54 refers to the active section of the modulator, to characterize the propagation losses of this part. Thus, the whole modulator electrode (inset of Figure 60-a) shows a slightly increase of the propagation losses. Furthermore, the mismatch between optical and microwave indices also contributes to the reduction of the bandwidth but it is not taken into account in the scattering parameters measurement. Figures 60-b, 60-c and 60-d shows measured signals at 10,15 and $30 \mathrm{GHz}$ where the blue wave corresponds to the optical modulated signal and the pink wave to the electrical modulating one. At higher frequencies, the electrical signals are attenuated due to the electrode losses, which lead to a lower modulation effect. Nonetheless, the 
demonstration of EO modulation at frequencies beyond $10 \mathrm{GHz}$ discards the presence of any other effect rather than Pockels as responsible of the modulation.
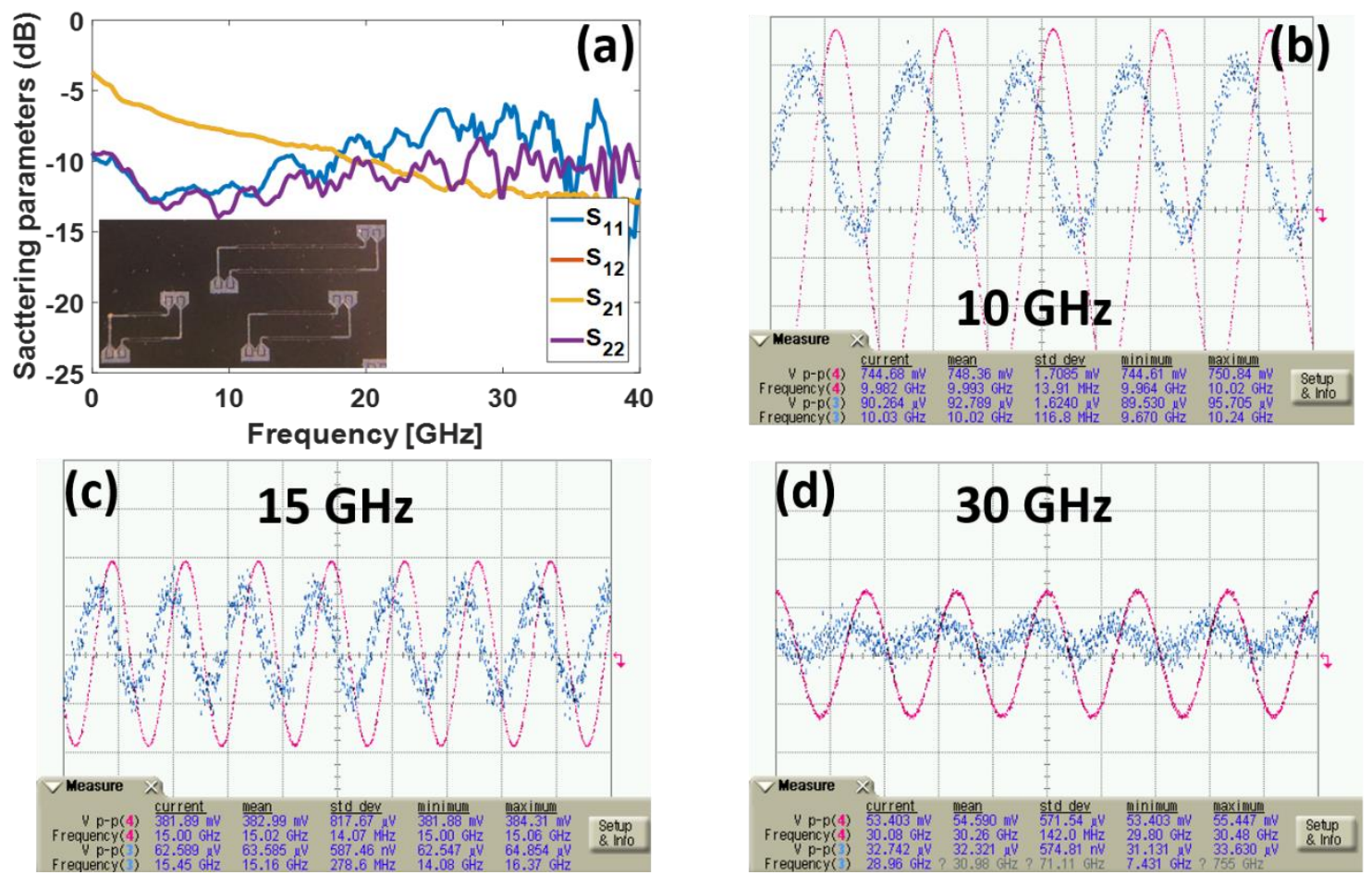

Figure 60. (a) Scattering parameters of the electrodes modulator with an inset of modulator electrodes of different lengths, (b) modulated (blue) and modulating (pink) signal at $10 \mathrm{GHz}$, (c) at $15 \mathrm{GHz}$ and (d) at $30 \mathrm{GHz}$.

Finally, high-speed digital modulation has been accomplished with a bit rate up to 10 Gbps. Therefore, Figure 61-a shows an eye diagram for a bit rate of 5 Gbps and Figure 61-b shows the maximum bit rate achieved for the modulator: 10 Gbps.
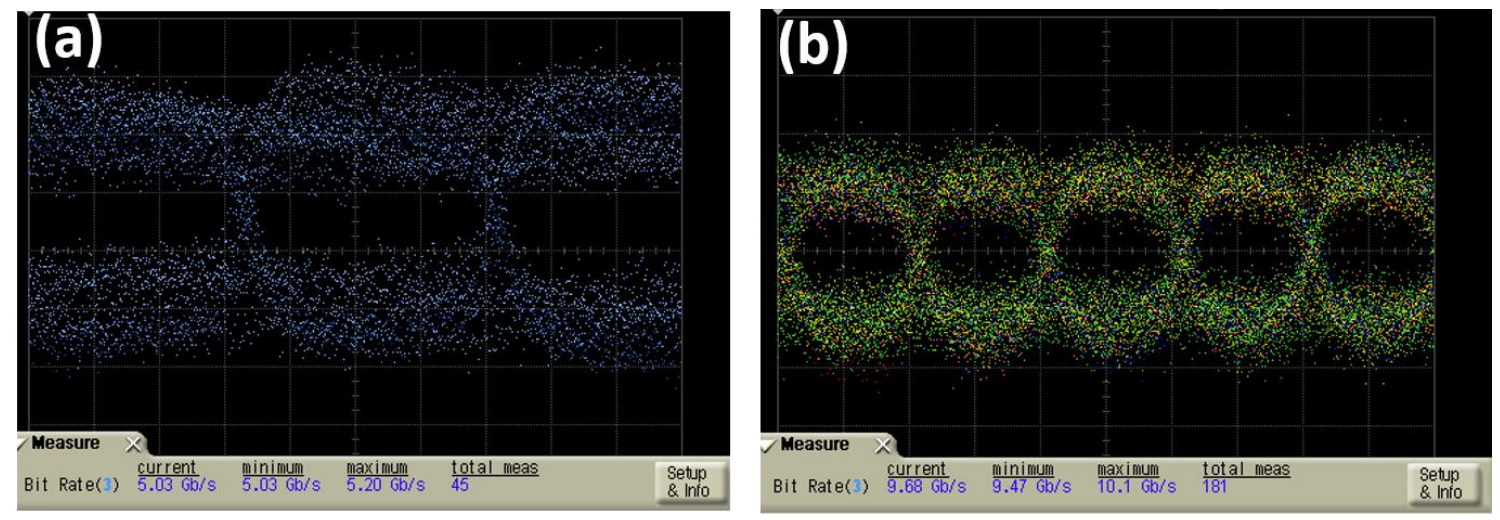

Figure 61. (a) eye diagram obtained from the digital characterization of the modulator at 5 Gbps, and (b) eye diagram at 10 Gpbs. 


\subsection{Conclusions}

In this chapter, the multi-line method measurement combined with electromagnetic simulations has been carried out to obtain and demonstrate the relative permittivity of BTO thin films deposited on top of a SOI substrate. In such a way, the permittivity can be obtained at wafer level and at the same process conditions used for fabricating the modulator devices. Similar values around 1200 at RF frequencies have been obtained for BTO grown by two different techniques: MBE and sputtering. The high permittivity value is associated with the c-axis ferroelectric domain orientation of the BTO layer, as it has been previously reported.

Furthermore, a coplanar strip-line electrode has also been designed and experimentally demonstrated for high speed modulation in a hybrid BTO/Si device. The modulation bandwidth is limited by the microwave propagation losses. Lower losses have been obtained by MBE compared to sputtering which suggest that process conditions could be optimized to minimize the dielectric losses of the BTO layer. Nevertheless, a theoretical study with the experimental values of losses and microwave indices has been carried out and modulation bandwidths up to $40 \mathrm{GHz}$ have been demonstrated to be feasible.

In the last step, electro-optical characterization of the fabricated modulator has been carried out. Only the MBE grown BTO modulator has been able to be characterized. The high microwave propagation losses of sputtering grown BTO and the amorphous regions avoid the modulation effect. However, MZI modulator with MBE grown BTO has produced good results with an impedance around 50 $\Omega$, low propagation losses and a modulation bit rate up to $10 \mathrm{Gbps}$. 


\section{Chapter 5}

\section{Conclusions and future outlook}

This work has contributed to the development of optoelectronic silicon photonics devices with enhanced performance by focusing on the design and optimization of the electrical parts. Highly efficient micro-heaters allow to take advantage of the high thermo-optic coefficient of silicon or the metal-to-insulator transition of the vanadium dioxide for developing low power consumption photonic switches. A trade-off between power consumption and switching time usually occurs, which is mainly imposed by the physical effect used to change the effective index of the optical mode. Nevertheless, some improvements can be carried out in order to reduce the power consumption. For instance, doping the silicon part of the MMI, which is going to be heated-up, to make this part work as the high resistant of the circuit. This will help to concentrate the heat in the area of interest although the cooling time will become slower. In this way, the characteristics of the design will remain on the requirements of the application. Regarding to vanadium dioxide, improvements in the fabrication process to induce the insulator-to-metal transition with lower temperatures will contribute to reduce the power consumption and eventually provide faster switching times. The impact of the film quality on the switching performance remains also as an interesting point to be addressed.

With respect to the slow-wave electrodes, a high microwave index of 11.6 has been demonstrated up to $40 \mathrm{GHz}$ in coplanar waveguides. The work developed in chapter 3 opens the door for new designs of electro-optic modulators based on the slow-wave phenomenon, matching the microwave and optical indices for maximizing the bandwidth, and allowing the reduction of the modulator footprint. 
Furthermore, slow-wave coplanar waveguides could play a key role in other fields like microwave devices, plasmonics or metamaterials. As this thesis delves on the design of the electrodes to enhance the performance of optoelectronic devices, it looks natural that the future outlook for this thesis should be focused on the demonstration of a full slow-wave modulator.

Finally, the permittivity of barium titanate thin films grown on SOI wafers has been obtained in order to properly design the electrodes of modulators. A $50 \Omega$ electrode design has been carried out with good agreement between simulation results and measurements. Scattering parameters and microwave propagation losses have been obtained for both grown methods of the BTO layer; RF sputtering and MBE. Higher microwave losses have been measured for sputtered grown BTO. However, MBE grown BTO allowed to fabricate and characterize a MZI modulator reaching bit rates up to $10 \mathrm{Gbps}$, one of the fastest bit rates for a BTO/Si based MZI modulators to date. Future improvements of BTO/Si modulators should be focused on the optimization of the fabrication processes to reduce microwave losses. The influence of the quality of the $\mathrm{SrTiO}_{3} / \mathrm{BaTiO}_{3}$ interface on the microwave propagation losses could be investigated. New MZI configurations with slow-wave electrodes could also be foreseen to reduce the footprint without penalizing the electro-optical bandwidth. Ultimately, another important issue that is not demonstrated yet on BTO is the electro-optical bistability. Bistability could open a new path on the design of new photonic devices such as optical memories or ultra-fast and low-power consumption switches. 


\section{List of publications}

\section{a. Journals}

- Á. Rosa, A. Gutiérrez, A. Brimont, A. Griol, and P. Sanchis, "High performace silicon $2 \times 2$ optical switch based on a thermo-optically tunable multimode interference coupler and efficient electrodes," Opt. Express 24, 191 (2016).

- L. D. S. Diana, F. C. Juan, A. R. Escutia, and P. S. Kilders, "Ultra-compact electro-absorption VO2-Si modulator with TM to TE conversion," J. Opt. (United Kingdom) 19, 35401 (2017).

- L. Sánchez, A. Rosa, A. Griol, A. Gutierrez, P. Homm, B. Van Bilzen, M. Menghini, J. P. Locquet, and P. Sanchis, "Impact of the external resistance on the switching power consumption in VO 2 nano gap junctions," Appl. Phys. Lett. 111, 31904 (2017).

- Á. Rosa, D. Tulli, P. Castera, A. G. Ana M. Gutierrez, B. V. Mariano Baquero, F. Eltes, S. Abel, J. Fompeyrine, and P. Sanchis, "Barium titanate (BaTiO3) RF characterization for application in electro-optic modulators," Opt. Mater. Express 7, 4328-4336 (2017).

- Á. Rosa, S. Verstuyft, A. Brimont, D. Van Thourhout, and P. Sanchis, "Microwave index engineering for slow-wave coplanar waveguides," Sci. Rep. 8, $5672(2018)$. 
- S. Abel, F. Eltes, J. E. Ortmann, A. Messner, P. Castera, T. Wagner, D. Urbonas, A. Rosa, A. M. Gutierrez, D. Tulli, P. Ma, B. Baeuerle, A. Josten, W. Heni, D. Caimi, A. A. Demkov, J. Leuthold, P. Sanchis, J. Fompeyrine, "Large Pockels effect in micro- and nano-structured barium titanate integrated on silicon," Nature Materials (Accepted, August 2018).

- Castera, P., Gutierrez, A. M., Rosa, A., Tulli, D., Griol, A., Angelova, T., Bellieres, L., Hurtado, L., Eltes, F., Abel, S., Fompeyrine, J., \& Sanchis, P. "High speed electro-optic Mach-Zehnder modulator based on a hybrid BaTiO3/Silicon waveguide structure," To be submitted.

- A. Brimont, A. Zanzi, C. Vagionas, A. Griol, A. Rosa, S. Lechago, J. Hurtado, L. Bellieres, N. S. Losilla, N. Pleros, C. Vyrsokinos, J. Kraft, V.Sidorov, T.Tekin, J. Marti and P. Sanchis, "Alignment Tolerant, Low Voltage, 0.26 V.cm, Push-Pull Silicon Photonic Switches Based on a Highly Doped Vertical pn Junction", to be submitted.

\section{b. Conferences}

- Á. Rosa, A. M. Gutiérrez, A. Brimont, A. Aamer, and P. Sanchis, "A siliconbased tunable multimode interferometer using the thermo-optic effect," in 16th European Conference on Integrated Optics (2012), pp. 4-5.

- Á. Rosa, A. Brimont, A. Griol, and P. Sanchis, "Optimized micro-heater structures for tunable silicon multimode interferometers," in IEEE International Conference on Group IV Photonics GFP (2014), Vol. 26, pp. $91-92$.

- Á. Rosa, A. M. Gutierrez, A. Brimont and P. Sanchis, "Design of lumped electrodes for silicon Mach-Zehnder Interferometer switches," in 4th Conferencia española de Nanofotónica - CEN (2014-05-14 - 2014-05-16). Santander (Spain).

- M. Riquelme, D. Ortiz, L. Sánchez, A. Rosa, A. Griol, P. Sanchis and J. Martí, "Graphene-based uniform heat spreader for silicon photonic devices," in XXX Trobades Científiques de la Mediterrània Josep Miquel Vidal (201411-10 - 2014-11-17). Mahón (Spain). 
- Á. Rosa, A. Griol, A. M. Gutiérrez, A. Brimont, and P. Sanchis, "Silicon 2x2 Optical Switch Based on Optimized Multimode Interference Coupler to Minimize Power Consumption," Eur. Conf. Opt. Commun. 3-5 (2015).

- P. Castera, A. Rosa, A. M. Gutierrez, D. Tulli and P. Sanchis, "Optimization of a hybrid BaTiO3/Si Waveguide Structure for Electro-optic Modulation." in 5th Conferencia Española de Nanofotónica - CEN (2016-06-20 - 2016-0622). Valencia (Spain).

- C. Op De Beeck, A. Rosa, L. D. Sanchez, J. Hurtado, P. Sanchis, and C. De Vera, "Integration of indium tin oxide on silicon for enabling electro-optical functionalities," in 5th Conferencia Española de Nanofotónica - CEN (201606-20 - 2016-06-22). Valencia (Spain).

- L. D. Sánchez, A. Rosa, T. Angelova, J. Hurtado, A. Griol, P. Sanchis, M. Menghini, P. Homm, B. Van Bilzen, J. P. Locquet, and L. Zimmermann, "Electrical switching in hybrid VO2/Si photonic structures," in International Conference on Transparent Optical Networks (IEEE, 2016), Vol. 2016Augus, pp. 1-4.

- P. Castera, A. Rosa, D. Tulli, A. M. Gutierrez, S. Cueff, R. Orobtchouk, P. R. Romeo, G. Saint-Girons, and P. Sanchis, "Towards high-speed electrooptical performance in a hybrid BaTiO3/Si Mach-Zehnder modulator," in IEEE International Conference on Group IV Photonics GFP (IEEE, 2016), Vol. 2016-Novem, pp. 54-55.

- P. Castera, A. M. Gutierrez, A. Rosa, D. Tulli, and P. Sanchis, "Optimization of a BaTiO3/Si Waveguide Structure for Electro-optic Modulation," in V Conferencia Española de Nanofotónica (CEN 2016) (2016).

- S. Cueff, P. Castera, A. M. Gutierrez, P. R. Romeo, R. Orobtchouk, B. Wague, B. Vilquin, P. Regreny, A. Rosa, T. Angelova, A. Griol, P. Sanchis, and G. Saint-Girons, "Hybrid silicon-ferroelectric oxide platform for tunable nanophotonics on silicon," in International Conference on Transparent Optical Networks (IEEE, 2016), Vol. 2016-Augus, pp. 1-4.

- L. Sanchez, A. Rosa, A. Griol, M. Menghini, P. Homm, B. Van Bilzen, J.-P. Locquet, C. Mai, L. Zimmermann, and P. Sanchis, "Thermo-optical switching in hybrid VO2/Sİ waveguides by lateral displaced microheaters," 2017 IEEE 14th Int. Conf. Gr. IV Photonics 41-42 (2017). 
- Zanzi, A. Rosa, A. Griol, P. Sanchis, J. Marti, and A. Brimont, "Advanced High Speed Slow-Light Silicon Modulators in the O-Band for Low Power Optical Interconnects in Data Centers," 2017 IEEE 14th Int. Conf. Gr. IV Photonics 4-5 (2017).

- Zanzi, A. Rosa, A. Griol, P. Sanchis, J. Marti, and A. Brimont, "Efficient and Optimized $25 \mathrm{~Gb} / \mathrm{s}$ Slow-Light SOI Modulator in the O-Band with Slowwave Electrodes for Low Power Optical Interconnects," in 20th European Conference on Integrated Optics (2018)-(Accepted). 


\section{References}

[1] Web page ITU (International Union of Telecommunications): http://www.itu.int/en/ITU-D/Statistics/Pages/facts/default.aspx

[2] M. J. O'Mahony, C. Politi, D. Klonidis, R. Nejabati and D. Simeonidou, "Future Optical Networks," in Journal of Lightwave Technology, vol. 24, no. 12, pp. 4684-4696, Dec. 2006. doi: 10.1109/JLT.2006.885765

[3] H. Subbaraman, X. Xu, A. Hosseini, X. Zhang, Y. Zhang, D. Kwong, and R. Chen, "Recent advances in silicon-based passive and active optical interconnects," Opt. Express 23, 2487-2511 (2015).

[4] B. Jalali and S. Fathpour, "Silicon photonics," J. Light. Technol. 24, 46004615 (2006).

[5] L. Vivien, A. Polzer, D. Marris-Morini, J. Osmond, J. M. Hartmann, P. Crozat, E. Cassan, C. Kopp, H. Zimmermann, and J. M. Fédéli, "Zero-bias 40Gbit/s germanium waveguide photodetector on silicon," Opt. Express 20, 1096 (2012).

[6] Fang, A. W., Park, H., Cohen, O., Jones, R., Paniccia, M. J., and Bowers, J. E., "Electrically pumped hybrid AlGaInAs-silicon evanescent laser," Optics express, 14(20), 9203-9210, (2006).

[7] B. Ben Bakir, A. Descos, N. Olivier, D. Bordel, P. Grosse, E. Augendre, L. Fulbert, and J. Fedeli, "Electrically driven hybrid Si/III-V Fabry-Pérot lasers based on adiabatic mode transformers," Opt. Express 19, 1031710325 (2011).

[8] P. Castera Molada, "Development of new photonic devices based on barium titanate in silicon," Universitat Politècnica de València (2017). 
[9] R. C. Alferness, "Waveguide Electrooptic Modulators," IEEE Trans. Microw. Theory Tech. 30, 1121-1137 (1982).

[10] R. Spickermann and N. Dagli, "Experimental analysis of millimeter wave coplanar waveguide slowwave structures on GaAs," IEEE Trans. Microw. Theory Tech. 42, 1918-1924 (1994).

[11] D. A. Motta, Y. R. R. Bustamante, A. P. Freitas, G. B. De Farias, U. C. Moura, and L. H. Gabrielli, "Design of a $40 \mathrm{GHz}$ Bandwidth Slow-Wave Silicon Modulator," 2017 SBMO/IEEE MTT-S Int. Microw. Optoelectron. Conf. 1-5 (2017).

[12] R. Spickermann, M. G. Peters, and N. Dagli, "A polarization independent GaAs-AlGaAs electrooptic modulator," IEEE J. Quantum Electron. 32, 764-769 (1996).

[13] A. Brimont, D. J. Thomson, P. Sanchis, J. Herrera, F. Y. Gardes, J. M. Fedeli, G. T. Reed, and J. Martí, "High speed silicon electro-optical modulators enhanced via slow light propagation," Opt. Express 19, 20876 (2011).

[14] G. T. Reed, G. Mashanovich, F. Y. Gardes, and D. J. Thomson, "Silicon optical modulators," Nat. Photonics 4, 518-526 (2010).

[15] A. Reed, Graham Knights, Silicon Photonics: An Introduction (John Wiley, 2013), Vol. 53 (2013).

[16] R. A. Soref and B. R. Bennett, "Electrooptical effects in silicon," IEEE J. Quantum Electron. 23, 123-129 (1987).

[17] J. P. Dakin and R. G. W. Brown, Handbook of Optoelectronics (Taylor \& Francis, 2006).

[18] H. Chung, W. S. C. Chang, and E. L. Adler, "Modeling and Optimization of Traveling-Wave LiNbO3 Interferometric Modulators," IEEE J. Quantum Electron. 27, 608-617 (1991).

[19] H. Subbaraman, X. Xu, A. Hosseini, X. Zhang, Y. Zhang, D. Kwong, and R. T. Chen, "Recent advances in silicon-based passive and active optical interconnects," Opt. Express 23(3), 2487-2510 (2015). 
[20] D. Nikolova, S. Rumley, D. Calhoun, Q. Li, R. Hendry, P. Samadi, and K. Bergman, "Scaling silicon photonic switch fabrics for data center interconnection networks," Opt. Express 23(2), 1159-1175 (2015).

[21] P. Dong, S. F. Preble, and M. Lipson, "All-optical compact silicon comb switch," Opt. Express 15(15), 9600-9605 (2007).

[22] A. Biberman, H. L. R. Lira, K. Padmaraju, N. Ophir, J. Chan, S. Member, M. Lipson, S. Member, and K. Bergman, "Broadband silicon photonic electrooptic switch for photonic interconnection networks," IEEE Photonics Technol. Lett. 23(8), 504-506 (2011).

[23] G. Li, X. Zheng, J. Yao, H. Thacker, I. Shubin, Y. Luo, K. Raj, J. E. Cunningham, and A. V. Krishnamoorthy, "25Gb/s 1V-driving CMOS ring modulator with integrated thermal tuning," Opt. Express 19(21), 2043520443 (2011).

[24] K. Tanizawa, K. Suzuki, M. Toyama, M. Ohtsuka, N. Yokoyama, K. Matsumaro, M. Seki, K. Koshino, T. Sugaya, S. Suda, G. Cong, T. Kimura, K. Ikeda, S. Namiki, and H. Kawashima, "Ultra-compact $32 \times 32$ strictlynon-blocking Si-wire optical switch with fan-out LGA interposer," Opt. Express 23, 17599 (2015).

[25] Y. Shoji, K. Kintaka, S. Suda, H. Kawashima, T. Hasama, and H. Ishikawa, "Low-crosstalk 2 x 2 thermo-optic switch with silicon wire waveguides.," Opt. Express 18, 9071-9075 (2010).

[26] P. Dong, S. Liao, H. Liang, R. Shafiiha, D. Feng, G. Li, X. Zheng, A. V. Krishnamoorthy, and M. Asghari, "Submilliwatt, ultrafast and broadband electro-optic silicon switches," Opt. Express 18(24), 25225-25231 (2010).

[27] P. Sun and R. M. Reano, "Submilliwatt thermo-optic switches using freestanding silicon-on-insulator strip waveguides," Opt. Express 18(8), 84068411 (2010).

[28] M. R. Watts, J. Sun, C. DeRose, D. C. Trotter, R. W. Young, and G. N. Nielson, "Adiabatic thermo-optic Mach-Zehnder switch," Opt. Lett. 38(5), 733-735 (2013). 
[29] N. C. Harris, Y. Ma, J. Mower, T. Baehr-Jones, D. Englund, M. Hochberg, and C. Galland, "Efficient, compact and low loss thermo-optic phase shifter in silicon," Opt. Express 22(9), 10487-10493 (2014).

[30] K. Suzuki, G. Cong, K. Tanizawa, S.-H. Kim, K. Ikeda, S. Namiki, and H. Kawashima, "Ultra-high-extinctionratio $2 \times 2$ silicon optical switch with variable splitter," Opt. Express 23(7), 9086-9092 (2015).

[31] L. Sanchez, A. Griol, S. Lechago, A. Brimont, and P. Sanchis, "Low-power operation in a silicon switch based on an asymmetric Mach-Zehnder interferometer," IEEE Photonics J. 7(2), 1-8 (2015).

[32] P. A. Besse, M. Bachmann, H. Melchior, L. B. Soldano, and M. K. Smit, "Optical bandwidth and fabrication tolerances of multimode interference couplers," J. Lightwave Technol. 12(6), 1004-1009 (1994).

[33] L. Soldano and E. Pennings, "Optical multi-mode interference devices based on self-imaging: principles and applications," J. Lightwave Technol. 13(4), 615-627 (1995).

[34] J. Leuthold and C. H. Joyner, "Multimode interference couplers with tunable power splitting ratios," J. Lightwave Technol. 19(5), 700-707 (2001).

[35] D. A. May-Arrioja and P. Likamwa, "Reconfigurable 3-dB MMI splitter," in Proc. IEEE/LEOS Summer Topical Meetings (IEEE, 2008), pp. 21-23.

[36] F. Wan, J. Yang, L. Chen, X. Jiang, and M. Wang, "Optical switch based on multimode interference coupler," IEEE Photonics Technol. Lett. 18(2), 421-423 (2006).

[37] Á. Rosa, A. Brimont, A. Griol, and P. Sanchis, "Optimized micro-heater structures for tunable silicon multimode interferometers," in Proc. IEEE Group IV Photonics (IEEE, 2014), pp. 91-92.

[38] Á. Rosa, A. Griol, A. M. Gutierrez, A. Brimont, and P. Sanchis, "Silicon $2 \times 2$ optical switch based on optimized multimode interference coupler to minimize power consumption," in European Conference on Optical Communications (2015), paper P.2.17. 
[39] Á. Rosa, A. Gutiérrez, A. Brimont, A. Griol, and P. Sanchis, "High performace silicon 2x2 optical switch based on a thermo-optically tunable multimode interference coupler and efficient electrodes," Opt. Express 24, $191(2016)$.

[40] A. Wasserman, Thermal Physics: Concepts and Practice (Cambridge University, 2012).

[41] Z. Abbas, R. D. Pollard, and R. W. Kelsall, "Determination of the Dielectric Constant of Materials from Effective Refractive Index Measurements," 47, 148-152 (1998).

[42] C. Ko and S. Ramanathan, "Observation of electric field-assisted phase transition in thin film vanadium oxide in a metal-oxide-semiconductor device geometry," Appl. Phys. Lett. 93, 1-4 (2008).

[43] A. Zimmers, L. Aigouy, M. Mortier, A. Sharoni, S. Wang, K. G. West, J. G. Ramirez, and I. K. Schuller, "Role of thermal heating on the voltage induced insulator-metal transition in $\mathrm{VO}_{2}, "$ Phys. Rev. Lett. 110, 1-5 (2013).

[44] M. A. Kats, R. Blanchard, P. Genevet, Z. Yang, M. M. Qazilbash, D. N. Basov, S. Ramanathan, and F. Capasso, "Thermal tuning of mid-infrared plasmonic antenna arrays using a phase change material," Opt. Lett. 38, 368 (2013).

[45] L. D. S. Diana, F. C. Juan, A. R. Escutia, and P. S. Kilders, "Ultracompact electro-absorption VO2-Si modulator with TM to TE conversion," J. Opt. (United Kingdom) 19, 35401 (2017).

[46] L. Sanchez, S. Lechago, A. Gutierrez, and P. Sanchis, "Analysis and Design Optimization of a Hybrid VO2/Silicon $2 \times 2$ Microring Switch," IEEE Photonics J. 8, (2016).

[47] A. Joushaghani, J. Jeong, S. Paradis, D. Alain, J. Stewart Aitchison, and J. K. S. Poon, "Wavelength-size hybrid Si-VO_2 waveguide electroabsorption optical switches and photodetectors," Opt. Express 23, 3657 (2015). 
[48] P. Markov, R. E. Marvel, H. J. Conley, K. J. Miller, R. F. Haglund, and S. M. Weiss, "Optically Monitored Electrical Switching in $\mathrm{VO}_{2}, "$ ACS Photonics 2, 1175-1182 (2015).

[49] S. Kaushal and B. K. Das, "Modeling and experimental investigation of an integrated optical microheater in silicon-on-insulator," Appl. Opt. 55, (2016).

[50] L. Sanchez, A. Rosa, A. Griol, M. Menghini, P. Homm, B. Van Bilzen, J. P. Locquet, C. Mai, L. Zimmermann, and P. Sanchis, "Thermo-optical switching in hybrid VO2/Sİ waveguides by lateral displaced microheaters," in 14th International Conference on Group IV Photonics, GFP 2017 (IEEE, 2017), pp. 41-42.

[51] I. Wolff, Coplanar Microwave Integrated Circuits (Wiley-Interscience, 2006).

[52] D. M. Pozar, "Microwave engineering," Addison-Wesley Publ. Co. (1993).

[53] J. Selga, P. Velez, J. Bonache, and F. Martin, "High miniaturization potential of slow-wave transmission lines based on simultaneous inductor and capacitor loading," in 2017 47th European Microwave Conference (EuMC) (IEEE, 2017), pp. 783-386.

[54] F. Wu and L. Sun, "Miniaturization of $4 \times 4$ Butler matrix using high slowwave factor structure," in 2017 IEEE 2nd Advanced Information Technology, Electronic and Automation Control Conference (IAEAC) (IEEE, 2017), pp. 1772-1775.

[55] J. Naylor, T. Weller, M. Smith, and J. Culver, "Slow-wave CPW slot-line transition," IEE Proc. - Microwaves, Antennas Propag. 152, 297 (2005).

[56] W. Hui and S. Li, "New Design of Delay Line Based on Slow-Wave Structure," in 2017 4th International Conference on Information Science and Control Engineering (ICISCE) (IEEE, 2017), pp. 1556-1558.

[57] B. Yang, H. J. Qian, Y. Shu, and X. Luo, "Compact bandpass filter with wide stopband using slow-wave CPW resonator with back-to-back coupledscheme," in 2017 IEEE International Symposium on Radio-Frequency Integration Technology (RFIT) (IEEE, 2017), pp. 13-15. 
[58] C.-Y. Kuo, A. Y.-K. Chen, C.-M. Lee, and C.-H. Luo, "Miniature 60 GHz slow-wave CPW branch-line coupler using 90 nm digital CMOS process," Electron. Lett. 47, 924 (2011).

[59] A. L. Franc, E. Pistono, G. Meunier, D. Gloria, and P. Ferrari, "A lossy circuit model based on physical interpretation for integrated shielded slowwave CMOS coplanar waveguide structures," IEEE Trans. Microw. Theory Tech. 61, 754-763 (2013).

[60] M. Miao, R. Fang, X. Zhang, B. Ning, F. Mu, Z. Li, W. Xiang, and Y. Jin, "Micromachined cavity-based bandpass filter and suspended planar slow-wave structure for vacuum-microelectronic millimeter-wave/ $\mathrm{THz}$ microsystem embedded in LTCC packaging substrates," in Proceedings Electronic Components and Technology Conference (IEEE, 2015), Vol. 2015-July, pp. 1767-1773.

[61] J. Sor, Y. Qian, and T. Itoh, "Miniature low-loss CPW periodic structures for filter applications," in IEEE Transactions on Microwave Theory and Techniques (2001), Vol. 49, pp. 2336-2341.

[62] L. Ye, Y. Xiao, Y. Liu, L. Zhang, G. Cai, and Q. H. Liu, "Strongly Confined Spoof Surface Plasmon Polaritons Waveguiding Enabled by Planar Staggered Plasmonic Waveguides," Sci. Rep. 6, 38528 (2016).

[63] X. L. Tang, Q. Zhang, S. Hu, Y. Zhuang, A. Kandwal, G. Zhang, and Y. Chen, "Continuous Beam Steering Through Broadside Using Asymmetrically Modulated Goubau Line Leaky-Wave Antennas," Sci. Rep. 7, (2017).

[64] A. Kianinejad, Z. N. Chen, and C. W. Qiu, "Design and modeling of spoof surface plasmon modes-based microwave slow-wave transmission line," IEEE Trans. Microw. Theory Tech. 63, 1817-1825 (2015).

[65] G. V. Eleftheriades, O. Siddiqui, and A. K. Iyer, "Transmission line models for negative refractive index media and associated implementations without excess resonators," IEEE Microw. Wirel. Components Lett. 13, $51-53(2003)$.

[66] C. Caloz and T. Itoh, Electromagnetic Metamaterials: Transmission Line Theory and Microwave Applications: The Engineering Approach (2005). 
[67] A. Grbic and G. V Eleftheriades, "Overcoming the Diffraction Limit with a Planar Left-Handed Transmission-Line Lens," Phys. Rev. Lett. 92, (2004).

[68] J. Shin, S. R. Sakamoto, and N. Dagli, "Conductor loss of capacitively loaded slow wave electrodes for high-speed photonic devices," J. Light. Technol. 29, 48-52 (2011).

[69] N. A. Jaeger and Z. K. Lee, "Velocity-matched slow-wave electrodes for integrated electro-optic modulators," in (1993), Vol. 1801, p. 965.

[70] S. Sakamoto, R. Spickermann, and N. Dagli, "Narrow gap coplanar slow wave electrode for travelling wave electro-optic modulators," Electron. Lett. 31, 1183-1185 (1995).

[71] A. L. Franc, D. Kaddour, H. Issa, E. Pistono, N. Corrao, J. M. Fournier, and P. Ferrari, "Slow-wave high performance shielded CPW transmission lines: A lossy model," in European Microwave Week 2009, EuMW 2009: Science, Progress and Quality at Radiofrequencies, Conference Proceedings - 39th European Microwave Conference, EuMC 2009 (2009), pp. 185-188.

[72] A. Bautista, A. L. Franc, and P. Ferrari, "Accurate Parametric Electrical Model for Slow-Wave CPW and Application to Circuits Design," IEEE Trans. Microw. Theory Tech. 63, 4225-4235 (2015).

[73] R. R. N. Simons, Coplanar Waveguide Circuits, Components, and Systems, Wiley Series in Microwave and Optical Engineering (John Wiley \& Sons, Inc., 2001), Vol. 7.

[74] H. Chen, "Development of an 80 Gbit / s InP-based Mach-Zehnder Modulator," (2007).

[75] A. Rosa, D. Tulli, P. Castera, A. G. Ana M. Gutierrez, B. V. Mariano Baquero, F. Eltes, S. Abel, J. Fompeyrine, and P. Sanchis, "Barium titanate (BaTiO3) RF characterization for application in electro-optic modulators," Opt. Mater. Express 7, 4328-4336 (2017).

[76] G. F. Engen and C. A. Hoer, "Thru-Reflect-Line: An Improved Technique for Calibrating the Dual Six-Port Automatic Network Analyzer," IEEE Trans. Microw. Theory Tech. 27, 987-993 (1979). 
[77] R. S. Jacobsen, K. N. Andersen, P. I. Borel, J. Fage-Pedersen, L. H. Frandsen, O. Hansen, M. Kristensen, A. V. Lavrinenko, G. Moulin, H. Ou, C. Peucheret, B. Zsigri, and A. Bjarklev, "Strained silicon as a new electrooptic material," Nature 441, 199-202 (2006).

[78] P. Damas, X. Le Roux, D. Le Bourdais, E. Cassan, D. Marris-Morini, N. Izard, T. Maroutian, P. Lecoeur, and L. Vivien, "Wavelength dependence of Pockels effect in strained silicon waveguides," Opt. Express 22, 2209522100 (2014).

[79] I. Olivares, T. Angelova, and P. Sanchis, "On the influence of interface charging dynamics and stressing conditions in strained silicon devices," Sci. Rep. 7, 7241 (2017).

[80] M. Borghi, M. Mancinelli, F. Merget, J. Witzens, M. Bernard, M. Ghulinyan, G. Pucker, and L. Pavesi, "High-frequency electro-optic measurement of strained silicon racetrack resonators," Opt. Lett. 40, 5287$5290(2015)$.

[81] A. Petraru, J. Schubert, M. Schmid, and C. Buchal, "Ferroelectric BaTiO3 thin-film optical waveguide modulators," Appl. Phys. Lett. 81, 1375-1377 (2002).

[82] P. Tang, D. J. Towner, T. Hamano, A. L. Meier, and B. W. Wessels, "Electrooptic modulation up to $40 \mathrm{GHz}$ in a barium titanate thin film waveguide modulator," Opt. Express 12, 5962 (2004).

[83] P. Tang, A. L. Meier, D. J. Towner, and B. W. Wessels, "BaTiO 3 thinfilm waveguide modulator with a low voltage-length product at nearinfrared wavelengths of 0.98 and 1.55 pm," Opt. Lett. 30, 254 (2005).

[84] M. J. Dicken, L. A. Sweatlock, D. Pacifici, H. J. Lezec, K. Bhattacharya, and H. A. Atwater, "Electrooptic modulation in thin film barium titanate plasmonic interferometers," Nano Lett. 8, 4048-4052 (2008).

[85] Jianheng Li, Zhifu Liu, Yongming Tu, Seng-Tiong Ho, Il Woong Jung, L. E. Ocola, and B. W. Wessels, "Photonic Crystal Waveguide Electro-Optic Modulator With a Wide Bandwidth," J. Light. Technol. 31, 1601-1607 (2013). 
[86] D. Sun, J. Zhang, C. Chen, M. Kong, J. Wang, and H. Jiang, "Theoretical Feasibility Demonstration for over $100 \mathrm{GHz}$ Electro-Optic Modulators with c-Axis Grown $\mathrm{BaTiO}_{3}$ Crystal Thin-Films," J. Light. Technol. 33, 1937-1947 (2015).

[87] P. Girouard, Z. Liu, P. Chen, Y. K. Jeong, Y. Tu, S.-T. Ho, and B. W. Wessels, "Enhancement of the pockels effect in photonic crystal modulators through slow light," Opt. Lett. 41, 5531 (2016).

[88] S. Abel, T. Stöferle, C. Marchiori, C. Rossel, M. D. Rossell, R. Erni, D. Caimi, M. Sousa, A. Chelnokov, B. J. Offrein, and J. Fompeyrine, "A strong electro-optically active lead-free ferroelectric integrated on silicon," Nat. Commun. 4, 1671 (2013).

[89] C. Xiong, W. H. P. Pernice, J. H. Ngai, J. W. Reiner, D. Kumah, F. J. Walker, C. H. Ahn, and H. X. Tang, "Active silicon integrated nanophotonics: Ferroelectric BaTiO3 devices," Nano Lett. 14, 1419-1425 (2014).

[90] F. Eltes, D. Caimi, F. Fallegger, M. Sousa, E. O'Connor, M. D. Rossell, B. Offrein, J. Fompeyrine, and S. Abel, "Low-Loss BaTiO3-Si Waveguides for Nonlinear Integrated Photonics," ACS Photonics 3, 1698-1703 (2016).

[91] W. H. P. Pernice, C. Xiong, F. J. Walker, and H. X. Tang, "Design of a silicon integrated electro-optic modulator using ferroelectric BaTiO3 Films," IEEE Photonics Technol. Lett. 26, 1344-1347 (2014).

[92] X. Hu, S. Cueff, P. R. Romeo, and R. Orobtchouk, "Modeling the anisotropic electro-optic interaction in hybrid silicon-ferroelectric optical modulator," Opt. Express 23, 1699 (2015).

[93] P. Castera, D. Tulli, A. M. Gutierrez, and P. Sanchis, "Influence of BaTiO3 ferroelectric orientation for electro-optic modulation on silicon," Opt. Express 23, 15332 (2015).

[94] S. Abel, T. Stöferle, C. Marchiori, D. Caimi, L. Czornomaz, M. Stuckelberger, M. Sousa, B. J. Offrein, and J. Fompeyrine, "A hybrid barium titanate-silicon photonics platform for ultra efficient electro-optic tuning," J. Light. Technol. 34, 1688-1693 (2016). 
[95] P. Castera, A. M. Gutierrez, D. Tulli, S. Cueff, R. Orobtchouk, P. R. Romeo, G. Saint-Girons, and P. Sanchis, "Electro-Optical Modulation Based on Pockels Effect in BaTiO3 with a Multi-Domain Structure," IEEE Photonics Technol. Lett. 28, 990-993 (2016).

[96] M. E. Lines and A. M. Glass, Principles and Applications of Ferroelectrics and Related Materials (Oxford University Press, 2001).

[97] D. Hennings, "Barium titanate based ceramic materials for dielectric use," Int. J. High Technol. Ceram. 3, 91-111 (1987).

[98] L. Sengupta and S. Sengupta, "Novel ferroelectric materials for phased array antennas," IEEE Trans. Ultrason. Ferroelectr. Freq. Control 44, 792797 (1997).

[99] F. De Flaviis, N. G. Alexopoulos, and O. M. Stafsudd, "Planar microwave integrated phase-shifter design with high purity ferroelectric material," IEEE Trans. Microw. Theory Tech. 45, 963-969 (1997).

[100] M. Zgonik, P. Bernasconi, M. Duelli, R. Schlesser, P. Günter, M. H. Garrett, D. Rytz, Y. Zhu, and X. Wu, "Dielectric, elastic, piezoelectric, electro-optic, and elasto-optic tensors of $\mathrm{BaTiO}_{3}$ crystals," Phys. Rev. B 50, 5941-5949 (1994).

[101] P. Tang, D. J. Towner, T. Hamano, A. L. Meier, and B. W. Wessels, "Electrooptic modulation up to $40 \mathrm{GHz}$ in a barium titanate thin film waveguide modulator," Opt. Express 12, 5962 (2004).

[102] P. Tang, A. L. Meier, D. J. Towner, and B. W. Wessels, "BaTiO3 thinfilm waveguide modulator with a low voltage-length product at nearinfrared wavelengths of 0.98 and 1.55 pm," Opt. Lett. 30, 254 (2005).

[103] M. J. Dicken, L. A. Sweatlock, D. Pacifici, H. J. Lezec, K. Bhattacharya, and H. A. Atwater, "Electrooptic modulation in thin film barium titanate plasmonic interferometers," Nano Lett. 8, 4048-4052 (2008).

[104] P. Girouard, Z. Liu, P. Chen, Y. K. Jeong, Y. Tu, S.-T. Ho, and B. W. Wessels, "Enhancement of the Pockels effect in photonic crystal modulators through slow light," Opt. Lett. 41, 5531 (2016). 
[105] C. Xiong, W. H. P. Pernice, J. H. Ngai, J. W. Reiner, D. Kumah, F. J. Walker, C. H. Ahn, and H. X. Tang, "Active silicon integrated nanophotonics: ferroelectric $\mathrm{BaTiO}_{3}$ devices," Nano Lett. 14, 1419-1425 (2014).

[106] M.-H. M. Hsu, A. Marinelli, C. Merckling, M. Pantouvaki, J. Van Campenhout, P. Absil, and D. Van Thourhout, "Orientation-dependent electro-optical response of $\mathrm{BaTiO}_{3}$ on $\mathrm{SrTiO}_{3}$-buffered $\mathrm{Si}(001)$ studied via spectroscopic ellipsometry," Opt. Mater. Express 7, 2030 (2017).

[107] P. Rabiei, J. Ma, S. Khan, J. Chiles, and S. Fathpour, "Heterogeneous lithium niobate photonics on silicon substrates," Opt. Express 21, 25573 (2013).

[108] W. H. P. Pernice, C. Xiong, F. J. Walker, and H. X. Tang, "Design of a silicon integrated electro-optic modulator using ferroelectric BaTiO3 Films," IEEE Photonics Technol. Lett. 26, 1344-1347 (2014).

[109] X. Hu, S. Cueff, P. R. Romeo, and R. Orobtchouk, "Modeling the anisotropic electro-optic interaction in hybrid silicon-ferroelectric optical modulator," Opt. Express 23, 1699 (2015).

[110] C. A. T. Siciunas, E. Salama, "Characteristics of RF Sputtered Barium Titanate Films on Silicon," J. Vac. Sci. Technol. 9, 91 (1972).

[111] T. Hayashi, N. Oji, and H. Maiwa, "Film thickness dependence of dielectric properties of $\mathrm{BaTiO}_{3}$ thin films prepared by sol-gel method," Jpn. J. Appl. Phys. 33, 5277-5280 (1994).

[112] T. Hamano, D. J. Towner, and B. W. Wessels, "Relative dielectric constant of epitaxial $\mathrm{BaTiO}_{3}$ thin films in the $\mathrm{GHz}$ frequency range," Appl. Phys. Lett. 83, 5274-5276 (2003).

[113] K. Schraml and D. Heberling, "Estimation of the propagation constant in multilayer microwave circuits using a low cost multiline system," in 2015 Loughborough Antennas and Propagation Conference, LAPC 2015 (2015), pp. 1-4.

[114] M. D. Janezic and J. a. Jargon, "Complex permittivity determination from propagation constant measurements," IEEE Microw. Guid. Wave Lett. 9, 76-78 (1999). 
[115] G. Ghione, Semiconductor Devices for High-Speed Optoelectronics (Cambridge University Press, 2009). 